\title{
A Schema for Interprocedural Modification Side-Effect Analysis with Pointer Aliasing
}

\author{
BARBARA G. RYDER \\ Rutgers University \\ WILLIAM A. LANDI \\ Siemens Corporate Research, Inc. \\ PHILIP A. STOCKS and SEAN ZHANG \\ Rutgers University \\ and \\ RITA ALTUCHER \\ Siemens Corporate Research, Inc.
}

\begin{abstract}
The first interprocedural modification side-effects analysis for $\mathrm{C}\left(\mathrm{MOD}_{C}\right)$ that obtains better than worst-case precision on programs with general-purpose pointer usage is presented with empirical results. The analysis consists of an algorithm schema corresponding to a family of $\mathrm{MOD}_{C}$ algorithms with two independent phases: one for determining pointer-induced aliases and a subsequent one for propagating interprocedural side effects. These $\mathrm{MOD}_{C}$ algorithms are parameterized by the aliasing method used. The empirical results compare the performance of two dissimilar $\mathrm{MOD}_{C}$ algorithms: $\operatorname{MOD}_{C}($ FSAlias $)$ uses a flow-sensitive, calling-context-sensitive interprocedural alias analysis; $\operatorname{MOD}_{C}$ (FIAlias) uses a flow-insensitive, calling-context-insensitive alias analysis which is much faster, but less accurate. These two algorithms were profiled on 45 programs ranging in size from 250 to 30,000 lines of $\mathrm{C}$ code, and the results demonstrate dramatically the possible costprecision trade-offs. This first comparative implementation of $\mathrm{MOD}_{C}$ analyses offers insight into the differences between flow-/context-sensitive and flow-/context-insensitive analyses. The analysis cost versus precision trade-offs in side-effect information obtained are reported. The results show surprisingly that the precision of flow-sensitive side-effect analysis is not always prohibitive in cost, and that the precision of flow-insensitive analysis is substantially better than worst-case estimates and seems sufficient for certain applications. On average $\mathrm{MOD}_{C}($ FSAlias $)$ for procedures and calls is in the range of $20 \%$ more precise than $\operatorname{MOD}_{C}($ FIAlias $)$; however, the performance was found to be at least an order of magnitude slower than $\operatorname{MOD}_{C}$ (FIAlias).
\end{abstract}

Categories and Subject Descriptors: D.3.4 [Programming Languages]: Processors-compilers; optimization; F.3.2 [Logics and Meanings of Programs]: Semantics of Programming Languages-program analysis

The research reported here was supported, in part, by Siemens Corporate Research and NSF grants CISE-CCR-9208632, CCR-9501761, GER-9023628.

Authors' address: W. A. Landi and R. Altucher, Siemens Corporate Research Inc, 755 College Rd. East, Princeton, NJ 08540; email: \{wlandi, raltucher\}@scr . siemens . com; B. G. Ryder, P. A. Stocks, and S. Zhang, Department of Computer Science, Rutgers University, 110 Frelinghuysen Road, Piscataway, NJ 08854; email: \{ryder, pstocks, xxzhang\}@cs .rutgers .edu.

Permission to make digital/hard copy of part or all of this work for personal or classroom use is granted without fee provided that the copies are not made or distributed for profit or commercial advantage, the copyright notice, the title of the publication, and its date appear, and notice is given that copying is by permission of the ACM, Inc. To copy otherwise, to republish, to post on servers, or to redistribute to lists, requires prior specific permission and/or a fee.

(C) 2001 ACM 0098-3500/01/0300-0105 $\$ 5.00$

ACM Transactions on Programming Languages and Systems, Vol. 23, No. 1, March 2001, Pages 105-186. 


\section{INTRODUCTION}

Accurate compile-time calculation of possible interprocedural side effects is crucial for aggressive compiler optimization [Aho et al. 1986], practical dependence analysis in programs with procedure calls [Banerjee 1988; Polychronopolous 1988; Wolfe 1989], data-flow-based testing [Bates and Horwitz 1993; Chatterjee and Ryder 1999; Frankel and Iakounenko 1998; Frankel and Weiss 1993; Harrold and Soffa 1991; Hutchins et al. 1994; Ostrand 1990; Weyuker 1994], incremental semantic change analysis of software [Burke 1990; Burke and Ryder 1990; Carroll and Ryder 1988; Cooper and Kennedy 1984; Marlowe and Ryder 1990a; 1991; Pollock and Soffa 1989; Ryder 1983; Ryder and Paull 1988; Yur et al. 1997; 1999], interprocedural def-use relations [Chatterjee 1999; Chatterjee and Ryder 1999; Ghiya and Hendren 1998; Harrold and Soffa 1994; Pande et al. 1994], and effective static interprocedural program slicing [Atkinson and Griswold 1996; 1998; Gallagher and Lyle 1991; Gupta and Soffa 1996; Harrold and Ci 1998; Horwitz et al. 1990; Larsen and Harrold 1996; Ottenstein and Ottenstein 1984; Reps and Rosay 1995; Sinha et al. 1999; Tip 1996; Tip et al. 1996; Tonella et al. 1997; Venkatesh 1991; Weiser 1984]. Many of these key applications in parallel and sequential programming environments need interprocedural def-use information. Interprocedural side-effect information can be used to approximate definitions; a similar calculation can approximate interprocedural variable uses. The utility of tools that address these problems can depend directly on the accuracy of the data-flow information available to them. Some problems may not need highly accurate data-flow information to solve them; in contrast, some applications may need to use all the information in a highly accurate solution. The latter applications need an efficient method to report program-point-specific side-effect information in the presence of pointers in order to handle modern languages such as $\mathrm{C}, \mathrm{C}^{++}$, FORTRAN90, and Java; ${ }^{1}$ this requires practical interprocedural side-effect analysis with pointers, something that previous techniques for FORTRAN cannot supply [Banning 1979; Burke 1990; Cooper 1985; Cooper and Kennedy 1987; 1988].

In the past, it has been suggested that one could do intraprocedural analyses of $\mathrm{C}$ codes, by using worst-case estimates of variables which could possibly experience a side effect at a call site. This yields a safe approximation of side-effect information, but almost surely overestimates the side effects in a program. To validate that useful program transformations can be applied, however, more accurate side-effect information may be needed.

This paper presents the first design and implementation of a schema for practical interprocedural modification side effects (i.e., $\mathrm{MOD}_{C}$ ) for languages

\footnotetext{
${ }^{1}$ Value-flow analysis for Java references is similar to pointer alias analysis [Chatterjee et al. 1999; Rountev et al. 2000]. 
with general-purpose pointers (e.g., C). ${ }^{2}$ Since determination of pointer-induced aliasing occurs in the schema as a separable phase, the schema actually represents a family of $\mathrm{MOD}_{C}$ algorithms. The empirical experiments reported involve two $\mathrm{MOD}_{C}$ methods with different component aliasing algorithms. $\operatorname{MOD}_{C}($ FSAlias $)$ uses a flow-sensitive, calling-context-sensitive approximation algorithm for pointer-induced aliasing, called FSAlias [Landi and Ryder 1992]; $\operatorname{MOD}_{C}($ FIAlias $)$ uses a flow-insensitive, calling-context-insensitive approximation algorithm for pointer-induced aliasing, called FIAlias, which is similar to the algorithm described in Zhang [1998] and Zhang et al. [1998]. The actually implemented algorithms handle unions and casting in $\mathrm{C}$ programs. The $\mathrm{MOD}_{C}$ schema is independent of the aliasing algorithm chosen and can use any aliasing algorithm, given a suitable interface. These $\mathrm{MOD}_{C}$ algorithms have extensive implementation results reported; these experiments are the first investigations of the cost-precision trade-offs of flow- and context-sensitivity as measured with respect to interprocedural side-effect analysis.

$\mathrm{MOD}_{C}($ FSAlias $)$ reports program-point-specific possible modification side effects; the results are more precise than information derivable using the same conservative alias summary for all statements of a procedure. After aliases are computed, they are used to gather procedure summary modification information categorized by calling context, with subsequent propagation of modifications through the program call multigraph. Finally, call site modification information is calculated using the results of the procedure side-effects summary. $\mathrm{MOD}_{C}($ FIAlias $)$ also reports program-point-specific possible modification side effects, but it uses alias information that is assumed to be valid globally throughout the program. Thus, more spurious side effects may be reported locally and propagated on the call multigraph (in a context-insensitive manner).

The empirical tests of these algorithms used $45 \mathrm{C}$ programs, most of which are publicly available. ${ }^{3}$ Measurements of average and maximum number of side effects found per assignment statement, per assignment through pointer dereference (i.e., a through-dereference assignment statement such as $* \mathrm{p}=$ ), per procedure, and per call have been recorded for both algorithms. Significantly, better precision is obtained by $\operatorname{MOD}_{C}(F S A l i a s)$ at greater time cost than $\operatorname{MOD}_{C}$ (FIAlias). This precision is necessary for some compiler transformations. $\operatorname{MOD}_{C}$ (FSAlias) shows surprising scalability on programs up to 10,000 lines of code at compile-time cost in the prototype. Extensive use of recursive data structures is a key factor that limits the scalability of $\operatorname{MOD}_{C}($ FSAlias $)$. Accordingly, $\operatorname{MOD}_{C}($ FSAlias $)$ successfully analyzes a 25,000-line program that does not use recursive data structures. Unexpectedly, $\operatorname{MOD}_{C}($ FIAlias $)$ is much more accurate than a coarse worst-case estimate and costs at least an order of magnitude less than $\operatorname{MOD}_{C}($ FSAlias $)$, so it may be sufficient and practical for program-understanding applications on large codes. The decreased cost is

${ }^{2}$ This is a presentation of the algorithm schema for $\mathrm{MOD}_{C}$ and describes new and extensive empirical results with two of the algorithms. The first $\mathrm{MOD}_{C}$ algorithm in the schema was discussed in Landi et al. [1993].

${ }^{3}$ Visit http://www.prolangs.rutgers.edu/ to obtain the public programs in this data set. 
primarily due to the cost of the FIAlias phase relative to FSAlias. In addition, several suggested improvements may augment the precision of $\operatorname{MOD}_{C}$ (FIAlias).

Specifically, the results for $\mathrm{MOD}_{C}$ (FSAlias) show that procedures modify on average 11 locations, while $\mathrm{MOD}_{C}($ FIAlias $)$ reports that procedures modify on average 17 locations, approximately $50 \%$ more side effects on average reported by the insensitive algorithm. ${ }^{4} \mathrm{~A}$ crude measure of the accuracy of $\operatorname{MOD}_{C}($ FIAlias $)$ versus $\mathrm{MOD}_{C}$ (FSAlias) can be obtained by examining the difference in their solutions on the data admitting both kinds of analysis, since both are safe estimates of side effects that can occur. Normalized differences at calls and for procedures are presented and discussed in Section 4.

The empirical results show the utility of both analyses for specific applications and demonstrate the precision gains from sensitivity for certain dataflow information. Recent work in partitioning programs for analyses [Ruf 1997; Zhang et al. 1996; 1998] yields hope that analyses of varying cost and precision can be applied to different parts of a program to obtain desired data-flow information at practical cost. The experiments reported here can be viewed as the initial investigation into the cost-precision trade-offs involved when using data-flow analyses of varying degrees of flow- and context-sensitivity.

This paper is organized as follows. Section 2 discusses issues of accurate interprocedural data-flow analysis and pointer aliasing algorithms. Section 3 presents the $\mathrm{MOD}_{C}$ algorithm schema, its worst-case complexity, and an example of both of the $\mathrm{MOD}_{C}$ algorithms used in the empirical tests. Section 4 reports the empirical results and derived observations. Section 5 details related work in data-flow analysis. Section 6 summarizes the contributions of the work. Appendix A presents a comparison of the MOD decomposition for $\mathrm{C}$ to that for FORTRAN. In Appendix B, an extended MOD algorithm based on our decomposition that approximately bounds the precision of side-effect solutions is explained. Finally, Appendix $\mathrm{C}$ presents the raw data from the empirical measurements discussed in Section 4.

\section{INTERPROCEDURAL DATA-FLOW ANALYSIS}

All interprocedural data-flow analyses for C-like languages encounter issues of problem formulation: how to obtain good static estimates of the possible execution paths through the program (including the possible calling patterns), how to treat variables created dynamically on the heap and aggregates (i.e., arrays, structs), and how to obtain good approximations to the possible aliasing induced by pointer usage in the program.

\subsection{Program Representation}

A program is represented by a common directed graph structure, an ICFG or interprocedural control flow graph. This is no more than the control flow graph of each procedure connected together at call sites, each of which has been split into a call node and a return node. Procedures are made to have a

${ }^{4}$ If data for moria, a statistical outlier, are removed, the average total for $\operatorname{MOD}_{C}($ FIAlias $)$ becomes 14. By contrast, removal of moria from the average total for $\operatorname{MOD}_{C}($ FSAlias $)$ has no effect.

ACM Transactions on Programming Languages and Systems, Vol. 23, No. 1, March 2001. 
single entry node and single exit node, if they do not already, by inserting extra (dummy) nodes and edges as required. Each call node is connected to the called procedure's entry node; each return node is connected to the called procedure's exit node. Figure 11 of Section 3.2 shows the ICFG representation of a small example program.

Iterative data-flow analysis is a fixed-point calculation for recursive equations defined on a graph representing a program that safely approximates the meet over all paths solution of a data-flow problem [Kildall 1973; Marlowe and Ryder 1990b]. For interprocedural data-flow analysis, not all paths in the usual graph representation correspond to real program executions. A realizable path is a path on which every procedure returns to the call site which invoked it [Jones and Muchnick 1982b; Landi and Ryder 1992; Reps et al. 1995; Sharir and Pnueli 1981]. Paths on which a procedure does not return to the call site which invoked it are unrealizable and can never happen in an actual execution. (setjump and longjump are not allowed in the $\mathrm{C}$ programs analyzed.) A fundamental problem of interprocedural analysis is how to restrict the propagation of data-flow information to realizable paths, especially when the data-flow functions are monotone rather than distributive so that the fixed-point solution need not be the meet-over-all-paths solution [Kam and Ullman 1977; Marlowe and Ryder 1990b].

\subsection{Issues Involving Variables}

The $\mathrm{MOD}_{C}$ schema defines a family of algorithms which determine modification side effects to fixed locations at program points. A fixed location is either a user-defined variable or a heap storage creation site. Each individual dynamically allocated fixed location is identified by the site that created it [Jones and Muchnick 1982b; Ruggieri and Murtagh 1988]; therefore, whereas two fixed locations created at the same allocation site are not distinguishable, those created at different sites are. Fixed locations are so named because the relation between a fixed location and the storage location to which it refers is unchanging during execution. Fixed locations do not contain any dereferences. For example in C syntax, $\mathrm{x}$ and $\mathrm{x} . \mathrm{f}$ are fixed locations. By contrast, for other $\mathrm{C}$ names which include dereferences (e.g., $* \mathrm{p}, \mathrm{p}->\mathrm{f}$ ), the relation between the name and the storage to which it refers can (and often does) change during execution. The term object name will be used to refer to all $\mathrm{C}$ names in general, with or without dereferences; thus fixed location will delineate a subset of those names. Side-effect information is obtained only for fixed locations.

All data-flow algorithms must deal with the a priori unbounded nature of recursive data structures. Many follow the approach of Jones and Muchnick [1982a] which limits, by truncation, the set of possible object names obtainable by following links in a recursive data structure, only maintaining the first $k$ dereferences, a process known as $k$-limiting. Others have suggested less naive ways of restricting the namespace while obtaining more accurate aliases of heap-stored objects [Chase et al. 1990; Deutsch 1994; Ghiya and Hendren 1996a; 1996b; Hendren and Nicolau 1990; Hendren et al. 1992; Horwitz et al. 1989; Larus and Hilfinger 1988; Sagiv et al. 1998]. 
One difficulty with k-limiting is that it loses information about the suffix of an object name. With 2-limiting, the alias $\langle p->f->g->h, x\rangle$ is represented by $\langle p->f->g \#, x\rangle$. However this k-limited object name also represents a set of object names, (e.g., $\mathrm{p}->\mathrm{f}->\mathrm{g}->\mathrm{h}_{i}$ for all $i$ that make sense, $*(\mathrm{p}->\mathrm{f}->\mathrm{g})$, $p->f->g->h->j$ ). If no casting is allowed, then type information can rule out most erroneous cases. However, in the presence of casting, especially when an algorithm (like FSAlias) attempts to handle the most aggressive instances (e.g., casting a pointer value to an integer and back again to pointer type), an algorithm using this kind of k-limiting will experience much imprecision.

The FSAlias algorithm uses k-limiting of names in recursive data structures, based on Jones and Muchnick's original k-limiting definition, combined with a naming scheme that identifies a dynamically created fixed location by its creation site. FIAlias needs no k-limiting because it only reports aliases involving those object names that explicitly appear in a program.

As in most pointer-aliasing algorithms, arrays are treated as single variables by FSAlias and FIAlias. Some algorithms distinguish the independent fields of a structure (e.g., FSAlias, FIAlias [Emami et al. 1994; Steensgaard 1996a; Wilson and Lam 1995; Zhang et al. 1996]) while others do not (e.g., Fahndrich et al. [2000], Foster et al. [2000], Das [2000], Shapiro and Horwitz [1997b], and Steensgaard [1996b]).

An added complication is presented by non-visible object names. The nonvisibles are local variables of procedures live at the call site (or in an earlier invocation of the current procedure) which are accessible through an alias, although not visible directly in the current scope. Possible side effects to these object names must be accounted for [Emami et al. 1994; Landi and Ryder 1992; Landi et al. 1993].

\subsection{Alias Representation}

The $\mathrm{MOD}_{C}$ schema requires knowledge of aliases, that is, object names that may refer to the same storage location at some point in the execution. In programming languages such as $\mathrm{C}$, explicit addressing operators render alias analysis more difficult than in FORTRAN, where aliases are introduced only through call-by-reference parameter passing. But the need for alias analysis still exists in modern programming languages whose pointer usage is more constrained (e.g., Java and FORTRAN90). ${ }^{5}$

Alias algorithms can be distinguished by their representation of the alias relations and the degree to which they preserve program-point-specific information. Aliases are either represented explicitly as pairs of object names or implicitly embedded in a points-to relation. ${ }^{6}$ Hendren et al. represent aliases as a set of simultaneous points-to relations at a particular program point [Emami et al. 1994; Hendren and Nicolau 1990] (e.g., $\langle\mathrm{x}, \mathrm{y}\rangle$ means $\mathrm{x}$ points to the object name y). Choi et al. use an implicit representation which stores all aliases as pairs

\footnotetext{
${ }^{5}$ The $\mathrm{MOD}_{C}$ schema would be largely unchanged for these languages although the alias phase would be specific to their simpler pointer usages.

${ }^{6}$ Differences between points-to analysis notation and the explicit pointer alias representation were also discussed in Emami et al. [1994].
}

ACM Transactions on Programming Languages and Systems, Vol. 23, No. 1, March 2001. 


$$
\begin{aligned}
& \text { S1: } x=\& y ; \\
& \text { S2: } \quad p=\& x ; \\
& \text { S3: } x=\& z ; \\
& \text { S4: } \quad * * p=
\end{aligned}
$$

(a) Implicit representation more precise

$$
\begin{array}{ll}
\text { S1: } & q=\& z ; \\
\text { S2: } & \text { if }() \\
\text { S3: } & p=\& q \\
& \text { else } \\
\text { S4: } & q=\& y ; \\
\text { S5: } & * * p=
\end{array}
$$

(b) Explicit representation more precise

$$
\begin{array}{ll}
\text { S1: } & \text { if }() \\
\text { S2: } & p=\& q ; \\
& \text { else } \\
\text { S3: } \quad r=\& s ; \\
\text { S4: } q=\& r ; \\
\text { S5: } \quad * * * p=
\end{array}
$$

(c) Both imprecise

Fig. 1. Comparison of implicit and explicit representations of aliases.

consisting of a fixed location and the object name (containing a single dereference) which points to it (e.g., $\langle * \mathrm{p}, \mathrm{x}\rangle$ means $\mathrm{p}$ points to $\mathrm{x}$ ). This representation, like a points-to representation, requires a closure step to obtain object names containing multiple levels of dereferences [Burke et al. 1994; 1997; Choi et al. 1993; Hind et al. 1999; Marlowe et al. 1993]. For example, in order to determine the fixed locations potentially experiencing side effects in $* * \mathrm{p}=$, the implicit representation pairs $\langle * \mathrm{p}, \mathrm{q}\rangle$ and $\langle * \mathrm{q}, \mathrm{r}\rangle$ must be combined to yield $\langle * * \mathrm{p}, \mathrm{r}\rangle$. FSAlias [Landi and Ryder 1992], the flow-/context-sensitive alias approximation algorithm, uses an explicit representation of aliases as pairs of object names possibly containing dereferences (e.g., $\langle * * \mathrm{p}, * \mathrm{q}\rangle$ ). Redundant aliases obtained through dereferences applied to both elements of an alias pair are not stored explicitly but are inferred (e.g., $\langle * \mathrm{p}, * \mathrm{q}\rangle$ implies $\langle * * \mathrm{p}, * * \mathrm{q}\rangle$ ). These two representations will be referred to by the terms explicit and implicit.

Essentially, the implicit representation is the same as that in the points-to relation, except the points-to has the "** implied for the first element, making the order of the relation relevant. By explicitly requiring the "*" to appear, the implicit representation can also directly represent reference parameter and structural aliases between object names, but not general aliasing (e.g., $\langle * * \mathrm{p}, * * \mathrm{q}\rangle$ ); all of these are directly representable with the explicit representation.

The implicit representation, sometimes referred to as compact, ${ }^{7}$ is claimed to be a space savings over the explicit representation, but no empirical or theoretical comparison has yet been made. The observed memory needs of the explicit representation used in FSAlias are bounded by the memory measurements in Section 4 . Thus, the memory trade-offs in choice of representation are not clear.

The implicit and explicit representations can be shown incomparable in terms of the resulting accuracy they exhibit as illustrated in Figure 1. In Figure 1(a), an algorithm using the implicit representation will result in the $\langle * x, z\rangle$ alias replacing incoming alias $\langle * \mathrm{x}, \mathrm{y}\rangle$ at statement $\mathrm{S} 3$; thus no aliases involving $\mathrm{y}$ will be reported at $\mathrm{S} 4$. By contrast, an algorithm using the explicit representation will have formed the alias $\langle * * \mathrm{p}, \mathrm{y}\rangle$ at statement $\mathrm{S} 2$, and it will remain in the alias set (as a spurious alias) reported at statement S4. In Figure 1(b), an algorithm using the implicit representation will combine the aliases $\langle * \mathrm{p}, \mathrm{q}\rangle$ and $\langle * \mathrm{q}, \mathrm{y}\rangle$ from opposite arms of the if statement and obtain the spurious alias $\langle * * \mathrm{p}, \mathrm{y}\rangle$ at $\mathrm{S} 5$, since this representation implies combination of any alias pairs

${ }^{7}$ The implicit representation is called compact in Burke et al. [1994; 1997], Choi et al. [1993], Hind and Pioli [1998], and Hind et al. [1999]. 
at a program point when aliases are needed. An algorithm using the explicit representation will not find this spurious alias because no transitive combination of alias pairs is required. In Figure 1(c), an algorithm using either the implicit or explicit representation will wrongly combine incoming aliases at statement $\mathrm{S} 5\langle * \mathrm{p}, \mathrm{q}\rangle$ and $\langle * \mathrm{r}, \mathrm{s}\rangle$ with the unconditional alias created at that statement, $\langle * \mathrm{q}, \mathrm{r}\rangle$, obtaining the spurious alias $\langle * * * \mathrm{p}, \mathrm{s}\rangle$. This occurs because neither representation encodes enough information to remember that the two incoming aliases do not exist concurrently on a path in the program. These examples are simple instances of the general problem.

\subsection{Analysis Precision and Sensitivity}

Since the basic problem of determining pointer-induced aliases is undecidable for programs with multiple levels of indirection [Landi 1992b; Landi and Ryder 1991; Ramalingam 1994], practical pointer-aliasing algorithms are approximate. Many algorithms use intraprocedural propagation of aliases through pointer-assignment statements in a manner conceptually similar to the singlelevel pointer-aliasing algorithm in Chapter 10 of Aho et al. [1986] with extensions to handle multiple-level pointers. Intraprocedural algorithms can make worst-case assumptions about the effects of call sites and determine a "first-cut" alias solution.

Most pointer aliasing algorithms now in the literature do some form of interprocedural analysis. They are distinguishable by the amount and type of calling context they preserve with the derived alias information. Some algorithms obtain differentiated program-point-specific alias information, because in these algorithms statement order of execution is significant. They are called flow-sensitive methods. When flow of control of execution is ignored, a flowinsensitive method is obtained. Algorithms which propagate alias information across calls, along paths in the called procedure, and then back again into the calling procedure, keeping approximate calling-context information with each alias pair, are termed context-sensitive, in that they distinguish back propagation of information between different call sites. Program-wide alias information is obtained by techniques which, upon identifying an alias, presume it holds throughout the program. This can be done in a context-sensitive or insensitive manner.

\subsection{Maintaining Calling Context}

There are many methods proposed for distinguishing calling contexts (i.e., the state of the call stack) in data-flow algorithms. Sharir and Pnueli [1981] advocate the use of a call-string list of open and not yet closed procedure activations to label data-flow information precisely with the calling context in which it was obtained. They also suggest use of an approximate call-string consisting of the last $j$ calls on the call stack. The call-string list is close to the approach used in the points-to algorithm developed at McGill University, where every procedure activation is analyzed separately [Emami et al. 1994; Hendren and Nicolau 1990]; optimizations to reduce computation by reusing the results for similar calling contexts were suggested by Emami [1993], and 
have been subsequently developed by Wilson and Lam [1995] for points-to analysis and Ghiya and Hendren [1996a] for connection analysis. Empirical data seems to suggest that such optimizations can dramatically reduce the number of contexts actually analyzed. Jones and Muchnick [1982b] describe the use of an abstraction of the calling context at a dynamic creation site for a variable; the precision of this abstraction plus the approximation lattice for the data-flow problem in question determine the precision of the solution. Choi et al. use the immediate past call site as their encoding of the calling context in their flow-sensitive aliasing algorithm [Burke et al. 1997; Choi et al. 1993; Hind et al. 1999; Marlowe et al. 1993]. They also describe an algorithm variant that uses alias sets of unrestricted size at the call site, called source alias sets, as additional call site encoding information. Their use of the previous call site name is the same as approximations suggested earlier by Jones and Muchnick [1982b] and Sharir and Pnueli [1981]. It also resonates with later work in the functional programming community on higher-order functions, Shivers' control flow analyses (CFA) [Shivers 1988] in which a suffix of the call stack contents is used to approximate calling context (e.g., 0CFA-no call sites distinguished, 1CFA-last call site distinguished, etc.).

The calling context approximation used in the $\mathrm{MOD}_{C}$ schema is inherited from the alias analysis used. For $\operatorname{MOD}_{C}($ FSAlias $)$, this is the same as that of the FSAlias algorithm [Landi and Ryder 1991; 1992]. The data-flow fact that $\mathrm{x}$ and y are aliased at program point $n$ is represented by an unordered pair $\langle\mathrm{x}, \mathrm{y}\rangle$ at $n$. The encoding of calling context is the set of reaching aliases ${ }^{8}(R A \mathrm{~s})$ that exists at entry of procedure $\mathrm{p}$ containing $n$ when $\mathrm{p}$ is invoked from a particular call site. When an alias exists independently of calling context, any reaching alias is an appropriate context to use, but to ensure correct propagation to all contexts and efficient representation, the special reaching alias $\phi$ is used. The $R A$ set can be used to determine to which call sites aliases at the exit of a called procedure should be propagated, namely only to those call sites which induce that $R A$ set. Essentially the $R A$ set induced by a call corresponds to a source alias set to which a namespace mapping is applied that includes the parameter bindings as well as scoping transformations. Using a single alias pair from the $R A$ set to determine calling context yields a safe approximate solution of realizable paths for programs containing multiple levels of dereferencing; this is the reaching alias $(R A)$ approximation used for calling context in $\operatorname{MOD}_{C}($ FSAlias $)$ at procedure entry. For aliasing in programs restricted to one level of dereferencing, the $R A$ sets are of cardinality one and can be used to obtain a precise solution [Landi and Ryder 1991]. The empirical results in Section 4 indicate that this is also a good approximation in practice. $R A$ is used in the description of the $\mathrm{MOD}_{C}$ schema to represent some approximation of calling context.

Figures 2 and 3 show that the reaching alias encoding of calling context is incomparable to using the last call site (i.e., 1CFA). For comparison purposes, these examples have been coded using the Landi-Ryder representation of aliases. In these examples aliases created independently of calling context are labeled with call site $\perp$ and spurious aliases are underlined.

${ }^{8}$ Reaching aliases were referred to by the term assumed aliases in Landi and Ryder [1992]. 


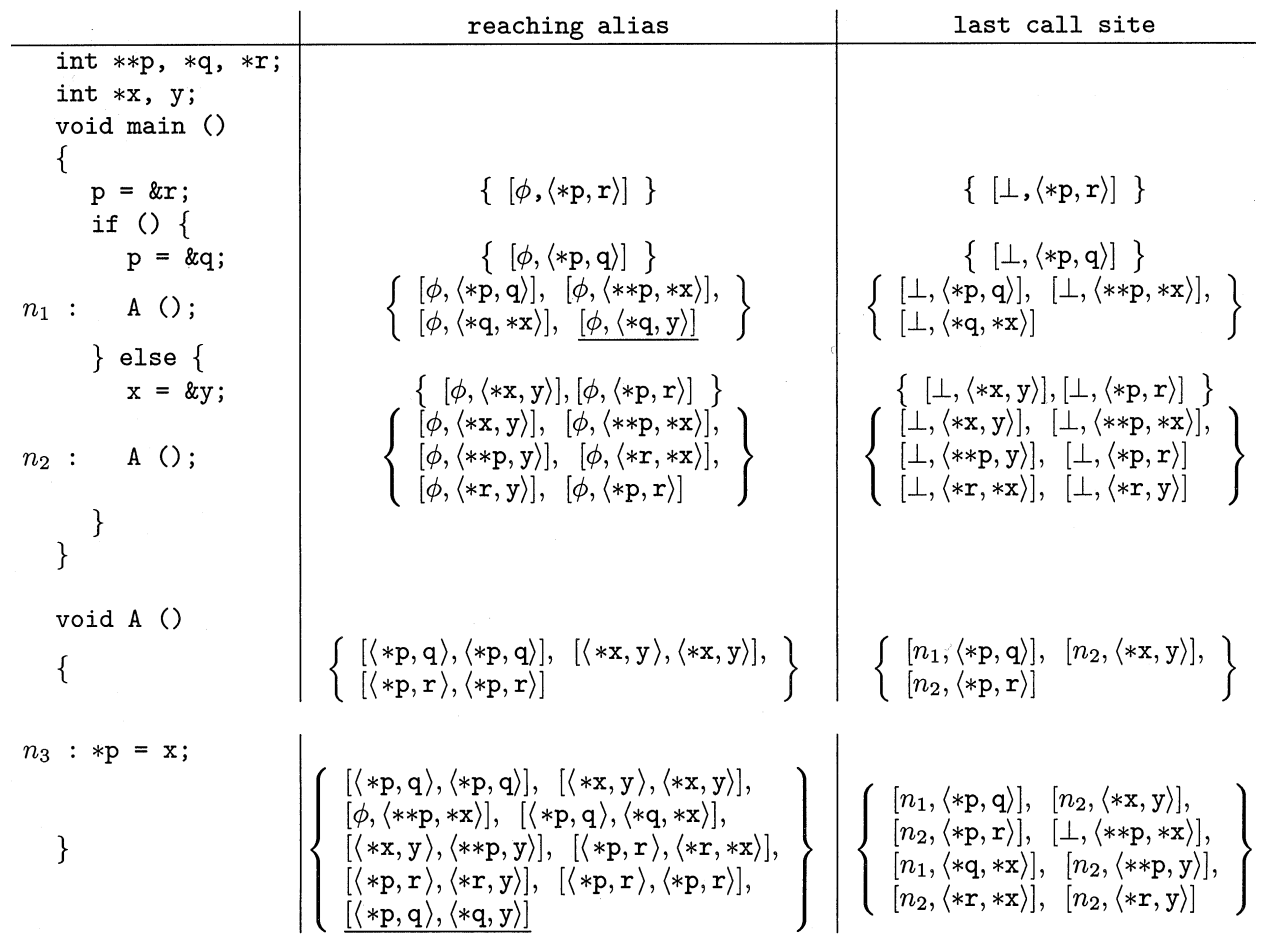

Fig. 2. An example in which last call site is more precise than reaching alias.

In Figure 2, the approximation arises because before $n_{3}$, an algorithm using reaching aliases for calling context cannot determine that $\langle * \mathrm{p}, \mathrm{q}\rangle$ and $\langle * \mathrm{x}, \mathrm{y}\rangle$ never occur on the same path; since different reaching aliases might correspond to the same call site, the safe approximation is to assume this does occur, which causes a spurious alias to be created. By using last call site information, however, an algorithm can see that each alias is labeled by a different call site. See Section 2.6.1 for further discussion of this example.

In Figure 3, the approximation in using last call site arises because on the return of $B$ to $A$, the algorithm has lost all information differentiating the call sites in the main program, whereas in this case, the reaching aliases distinguish the call sites; unlike in Figure 2, there is no approximation, since no aliases are created involving their possible interaction. Thus, these two calling-context approximations are incomparable.

\subsection{The FSAlias and FIAlias Algorithms}

The $\mathrm{MOD}_{C}$ schema inherits its calling-context sensitivity and flow sensitivity from the pointer-aliasing algorithm used. The empirical tests have exercised two specific choices of $\mathrm{MOD}_{C}$ algorithms at opposite ends of the sensitivity spectrum, namely $\operatorname{MOD}_{C}$ (FSAlias) which is flow-/context-sensitive, and $\mathrm{MOD}_{C}($ FIAlias $)$ which is flow-/context-insensitive. $\mathrm{MOD}_{C}($ FSAlias $)$ is more costly and more accurate, in general, than $\operatorname{MOD}_{C}$ (FIAlias) because of the 


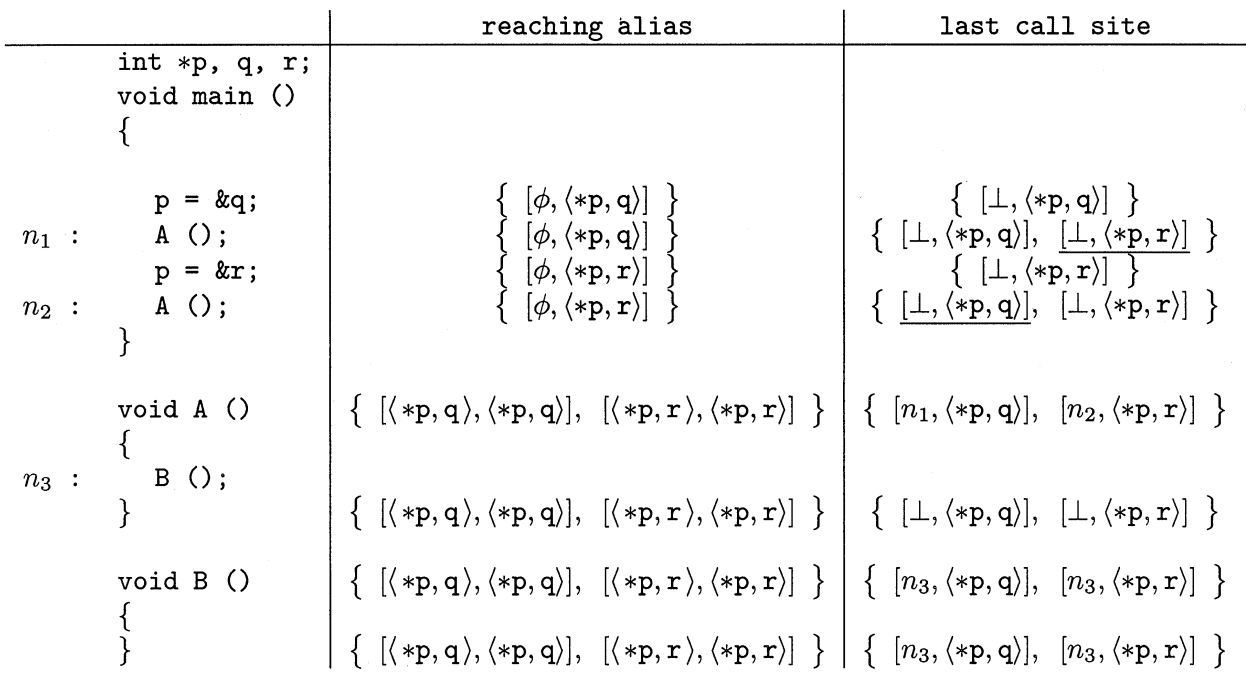

Fig. 3. An example in which reaching alias is more precise than last call site.

differences in cost and accuracy of the aliasing algorithms used. These techniques allow exploration of the precision/cost trade-offs of side-effects analysis and the scalability of these approaches applied to real programs. While the results obtained are specific to the two algorithms used, it seems likely that similar results will be obtainable from any pair of algorithms which vary similarly in sensitivity. More general claims would require additional experimentation.

The following alias algorithm descriptions are explained on an intuitive level to stress key concepts, with an example following to show the differences between FSAlias and FIAlias.

2.6.1 FSAlias. Figure 4 gives an overview of the FSAlias algorithm, which relies on a flow-/context-sensitive fixed-point iteration on the ICFG, using a standard worklist approach. In FSAlias, alias information is propagated along the static paths in each procedure for a specific calling context (i.e., a reaching alias) in a manner which preserves statement order; during this propagation, aliases are created or destroyed depending on the semantics of the program statements encountered on the path. Therefore, a full description of the algorithm requires a description of the transfer functions at intraprocedural nodes (i.e., pointer assignments) and at interprocedural nodes (i.e., call sites).

The initialization phase of the algorithm (step 1) populates the initial worklist with the initial set of aliases, either those created intraprocedurally by a pointer assignment (1.1) or interprocedurally by parameter-argument associations at calls (1.2). This is done by procedures alias_intro_by_assignment () and aliases_intro_by_call() respectively. Aliases that are created regardless of any reaching alias can legitimately be associated with any reaching alias; to ensure correct propagation to all contexts, they are only associated with a special reaching alias, $\phi$. 
begin

1. for each node $n$ in $I C F G$

1.1 if $n$ is a pointer assignment

1.2 else if $n$ is a call node

$$
\text { aliases_intro_by_assignment }(n) \text {; }
$$
aliases_intro_by_call $(n)$;

2. while worklist is not empty

2.1 remove $(n,[R A, P A])$ from worklist;

$/ *$ interprocedural propagation */

2.2 if $n$ is a call node alias_at_call_implies $(n, R A, P A)$;

2.3 else if $n$ is an exit node alias_at_exit_implies $(n, R A, P A)$;

2.4 else /* intraprocedural propagation * for each $m \in \operatorname{successor}(n)$

2.4.1 if $m$ is a pointer assignment alias_implies_thru_assign $(m, R A, P A)$;

\section{4 .2 else} preserve $(m, R A, P A)$;

end

Fig. 4. FSAlias algorithm.

Step 2 performs the data-flow information propagation both intraprocedurally and interprocedurally. During one step in the iteration, a (node, [calling context, alias]) entry is removed from the worklist, and propagated to successor nodes by invoking appropriate handling procedures. $(n,[R A, P A])$ represents the fact that alias $P A$ holds at node $n$ in calling context $R A$. The term alias tuple will be used to refer to an alias pair with its corresponding calling context (e.g., $[R A, P A]$ ). The intraprocedural propagation of aliases through pointer assignment statements is described by transfer functions associated with each node in a standard extension of Aho et al. [1986] (i.e., performed by procedure alias_implies_thru_assign() in step 2.4.1). If an intraprocedural node has no effect on pointer aliasing then the tuple is preserved through that node (i.e., performed by procedure preserve() in step 2.4.2).

For example, in Figure 9 at the exit of statement $8(\mathrm{p}=\mathrm{q})$ in main, the alias tuple $[\phi,\langle * \mathrm{p}, * \mathrm{q}\rangle]$ is generated under all calling contexts (i.e., 1.1 alias_intro_by_assignment ()$)$ The alias tuple $[\phi,\langle * \mathrm{q}, \mathrm{a}\rangle]$ reaches (and is preserved through) statement 8 after having been created at statement 7 . This alias is combined with the semantics of the pointer assignment at 8 to create alias $[\phi,\langle * \mathrm{p}, \mathrm{a}\rangle]$ at the exit of statement 8 . This fully describes the transfer function associated with the pointer assignment at statement 8 (i.e., 2.4.1 alias_implies_thru_assign()). Similar functions are used at all pointer assignments [Landi and Ryder 1992]. Essentially, an alias is created regardless of calling context by the assignment itself; current aliases of the left-hand-side object name (assuming it can be dereferenced) are killed by this assignment; and aliases of the right-hand-side object name (if any) become new aliases of the dereferenced left-hand-side object name. 
A more complex example of alias_implies_thru_assign() is offered at statement $n_{3}$ in Figure 2. Alias tuples [ $\left.\langle * \mathrm{p}, \mathrm{q}\rangle,\langle * \mathrm{p}, \mathrm{q}\rangle\right]$ and $[\langle * \mathrm{x}, \mathrm{y}\rangle,\langle * \mathrm{x}, \mathrm{y}\rangle]$ both reach statement $n_{3}$ and combine with the semantics of that statement to create alias $\langle * \mathrm{q}, \mathrm{y}\rangle$. Because two incoming alias tuples were needed, the algorithm arbitrarily choses one of their reaching aliases to associate with the created alias tuple $[\langle * \mathrm{p}, \mathrm{q}\rangle,\langle * \mathrm{q}, \mathrm{y}\rangle]$. This choice is arbitrary because the alias $\langle * \mathrm{q}, \mathrm{y}\rangle$ will only hold at call sites whose reaching alias set contains both $\langle * p, q\rangle$ and $\langle * \mathrm{x}, \mathrm{y}\rangle$. Some approximation may occur if there is a call site whose reaching alias set contains, for example, $\langle * \mathrm{p}, \mathrm{q}\rangle$ but not $\langle * \mathrm{x}, \mathrm{y}\rangle$, because given the above choice, $\langle * \mathrm{q}, \mathrm{y}\rangle$ will be propagated to that call site. Our empirical results indicate that this happens only infrequently.

Intuitively, the processing in step 2 for propagation of aliases across procedure boundaries occurs as follows. Interprocedurally, a call to procedure Q, $\operatorname{call}_{Q}$, creates reaching aliases at the entry of $Q$. If the algorithm is analyzing the calling procedure under calling context $R A$, contexts_of $\left(\right.$ call $\left._{Q}, R A\right)$ denotes the set of reaching aliases induced by both the parameter bindings (handled by step 1.2) and the aliases associated with $R A$ s which reach the call. The special reaching alias $\phi$ and reaching aliases created solely by the parameter bindings are included in the set contexts_of $\left(\operatorname{call}_{Q}, \phi\right) .{ }^{9} \operatorname{Alias}(n, R A)$ represents the set of aliases at program point $n$ under the calling context $R A$ which reaches the entry of the procedure containing $n .^{10}$ The mapping of $\operatorname{Alias}(n, R A)$ into the called procedure is handled by procedure alias_at_call_implies() in step 2.2 .

At the exit of $Q$, aliases associated with reaching alias $R A^{\prime}$ are propagated to any call site call $_{Q}$, where $R A^{\prime} \in$ contexts_of ( call $_{Q}, R A$ ), and thereafter are associated with $R A$ in the procedure containing that call site. This mapping is performed by procedure alias_at_exit_implies() in step 2.3. Aliases associated with reaching alias $\phi$ are valid at every call site. The details of the algorithm include the namespace mappings between the calling and called procedures [Landi and Ryder 1992].

For example, in Figure 9 the set of alias tuples which reach the call at statement 10 is $([\phi,\langle * \mathrm{p}, * \mathrm{q}\rangle],[\phi,\langle * \mathrm{q}, \mathrm{a}\rangle],[\phi,\langle * \mathrm{p}, \mathrm{a}\rangle])$. The transfer function at the call (i.e., 2.2 alias_at_call_implies()) maps each of these aliases into proc1 as a reaching alias, so that the alias tuples at exit of statement 3 in proc1 are $([\langle * \mathrm{p}, * \mathrm{q}\rangle,\langle * \mathrm{p}, * \mathrm{q}\rangle][\langle * \mathrm{q}, \mathrm{a}\rangle,\langle * \mathrm{q}, \mathrm{a}\rangle],[\langle * \mathrm{p}, \mathrm{a}\rangle,\langle * \mathrm{p}, \mathrm{a}\rangle])$. At the exit of statement 4 in proc1, we preserve all of these incoming alias tuples and generate new alias tuples: $([\phi,\langle * r, * \mathrm{q}\rangle],[\langle * \mathrm{q}, \mathrm{a}\rangle,\langle * \mathrm{r}, \mathrm{a}\rangle])$. Now, at the return from the call at statement 10 (recall that the ICFG breaks all call statements into two nodes, a call and a return), step 2.3 of the algorithm in Figure 4 (i.e., 2.3 alias_at_exit_implies()) propagates the alias tuples $([\phi,\langle * \mathrm{p}, * \mathrm{q}\rangle],[\phi,\langle * \mathrm{q}, \mathrm{a}\rangle],[\phi,\langle * \mathrm{p}, \mathrm{a}\rangle],[\phi,\langle * \mathrm{r}, * \mathrm{q}\rangle],[\phi,\langle * \mathrm{r}, \mathrm{a}\rangle])$, back to the return at statement 10 . Note that with respect to the last tuple, $R A$

\footnotetext{
${ }^{9}$ The correspondence between the non-visibles and non-addressable object names is memoized at the called procedure entry so that a local of a (possibly transitive) calling procedure is mapped to the representative object name $n v$ which is used throughout the alias propagation on paths from that procedure entry. Upon return from a call, any aliases involving $n v$ are expanded using the memoized information into the actual alias relations at the appropriate call sites.

${ }^{10}$ In Landi and Ryder [1992], may-holds were used to represent conditional aliasing information. $\operatorname{Alias}(n, R A)=\{P A \mid$ may-holds $(n, R A, P A)\}$.
} 
is $\phi$ in the previous discussion and $R A^{\prime}$ is $\langle * \mathrm{q}, \mathrm{a}\rangle$. This exemplifies the mapping of corresponding reaching aliases at call sites during alias propagation.

In Figure 9, statement 15 provides an example of 1.2 alias_intro_by_call(), which accounts for the implied assignments between corresponding actual arguments and formal parameters. Alias tuple $[\langle * \mathrm{f}, \mathrm{a}\rangle,\langle * \mathrm{f}, \mathrm{a}\rangle]$ is created at statement 15 and propagated to statement 16.

The main approximation which can occur in FSAlias is that sometimes two incoming aliases at a pointer assignment that are needed to create an alias as a side effect of that statement can never actually occur on the same execution path from program start to this assignment. There is an example of this in Figure 1(c). At the exit of statement $\mathrm{S} 4$, the existence of aliases $\langle * \mathrm{p}, \mathrm{q}\rangle$ and $\langle * \mathrm{r}, \mathrm{s}\rangle$ combined with the assignment at S4 seem to imply that $\langle * * * \mathrm{p}, \mathrm{s}\rangle$, but there is no execution path through the if statement on which both $\langle * \mathrm{p}, \mathrm{q}\rangle$ and $\langle * \mathrm{r}, \mathrm{s}\rangle$ hold. The assumption about incoming alias information is that sets of aliases can hold simultaneously on some path to this program point; this assumption may lead to safe but possibly imprecise aliases being created.

FSAlias has worst-case polynomial time complexity.

2.6.2 FIAlias. FIAlias is a fast coarse-grained alternative to FSAlias. FIAlias is a flow-/context-insensitive algorithm [Andersen 1994; Burke et al. 1994; Coutant 1986; Das 2000; Fahndrich et al. 2000; Foster et al. 2000; Guarna 1988; Hind and Pioli 1998; Rountev and Chandra 2000; Shapiro and Horwitz 1997b; Steensgaard 1996b; Weihl 1980; Zhang 1998; Zhang et al. 1996] which calculates the same points-to sets as Steensgaard's algorithm, although independently derived [Steensgaard 1996b; Zhang 1995]; we do not describe it as a nonstandard type analysis, and we do not use constraint-based solution procedures. Aliasing is expressed as a relation between pairs of object names, which is symmetric and reflexive, but not transitive; however, it is a common (though not universal) practice to approximate this relation with transitive solutions. FIAlias forms a partition of the object names which is a transitive representation of aliasing, and clearly can be represented with space linear in the number of object names in the program.

For simplicity of explication, assume (i) there are no structure assignments (these can be broken into multiple nonstructure assignments), (ii) all functions are of type void (there are many ways to handle returned values; for example, a function can be made to assign its return value to some new global, and that global can be used to retrieve the returned value at the call site), and (iii) there are no casting and no union types. (The handling of casting and union types is simply a matter of encoding the relationship of fields within object names [Yong et al. 1999].) Either fields can be represented by offsets from the start of the structure [Emami et al. 1994; Wilson and Lam 1995] or by symbolic names [Steensgaard 1996a; Zhang et al. 1996]. FIAlias uses the first approach.

The basic idea of FIAlias is that for the assignment $\mathrm{a}=\mathrm{b}, * \mathrm{a}$ and $* \mathrm{~b}$ become aliased (this is called a type 1 alias effect) and so do $*^{i} \mathrm{a}$ and $*^{i} \mathrm{~b}$ for all $i \geq 2$, assuming those object names make sense (this is called a type 2 alias effect). The type 2 alias effect induces the right-regular property discussed in Deutsch [1992] and Zhang et al. [1996]. 
The FIAlias algorithm partitions the program's object names using a union/find data structure [Aho et al. 1986]. An overview of the steps of FIAlias is as follows:

(1) Consider each object name to be in its own partition element.

(2) Perform unions of partition elements to account for type 1 alias effects at all assignments, with formal/actual bindings at call sites considered to be assignments.

(3) Perform unions of partition elements to account for type 2 alias effects.

(4) Perform some additional unions to ensure that actuals for function pointer call sites are unioned with the formals of the called procedure. When handling functions that are non-void, the returned value must be unioned with the name to which the value is assigned.

Typically the only alias information of interest concerns object names that physically appear in the program and their fixed-location aliases. The set of interesting object names is defined to be those object names needed to get an explicit alias solution for all object names that appear in the program and all fixed locations (i.e., including heap storage creation site names). If an object name $\mathrm{n}$ appears in an executable statement in the program, then $\mathrm{n}, * \mathrm{n}$, and any prefix of $\mathrm{n}$ are interesting; thus, if $\mathrm{p}->\mathrm{m}$ appears in the program, then $*(\mathrm{p}->\mathrm{m})$, $\mathrm{p}->\mathrm{m}$ and the prefixes $* \mathrm{p}$ and $\mathrm{p}$ are all interesting. Extending the interesting object names by one dereference is necessary to compute the aliases of $*$ p given $\mathrm{p}=\mathrm{q}$, since the aliases of $* \mathrm{q}$ must be known.

The FIAlias algorithm is presented in Figure 5. In Phase 1, an object name name is plausible if there is any possibility that $*$ name can have aliases; thus, constants are not plausible object names, and assignments like $i=5$ and $p=$ NULL generate no plausible left-hand-side or right-hand-side pairs. An assignment $i=j$ where $i$ and $j$ are integers is a more complicated case; this statement can generate the plausible pair $i / j$ if pointer-valued data are assumed to be transferable through integer object names using casts, as in the $\mathrm{C}$ program in Figure 6. Similarly, for $p=q+i$ where $p$ and $q$ are pointers and $i$ is an integer, $p / q$ must be considered as a plausible left-hand-side/right-hand-side pair, but $\mathrm{p} / \mathrm{i}$ may not be considered as such. The assignment function_pointer = function is treated as function_pointer = \&function for this analysis; that is, $*$ function_pointer must be unioned to function and not to $*$ function.

Phase 2 simply makes sure that for all pairs name ${ }_{i}$, name nan $_{j}$ in partition, that $*$ name $_{i}$ and $*$ name $_{j}$ are also in the same partition (i.e., type 2 alias effects). Phase 2 defines map so that for all object names $n$, map[find(n)][op] = find(op(n)) assuming $o p(n)$ is interesting. For example, map[find(n)][*] = find $(* n)$ if $* n$ is interesting. The type signature of map is

$$
\text { map : partition } \rightarrow \text { (operation } \rightarrow \text { partition }) .
$$

Map is built incrementally by iterating through all interesting object names. The merge function, also presented in Figure 5, unions two partitions and updates map. 
begin

/* Phase 1: Creating initial partitions */

for each plausible left-hand-side (lhs), right-hand-side (rhs) pair in the program union $(f i n d(* l h s)$, find $(* r h s))$

/* Phase 2: Refining partitions for Type 2 alias effects */

for each partition part

$\operatorname{map}[$ part $]=\lambda x$.NULL (everything maps to NULL)

for each interesting name $\mathrm{X}$ which is an operation $o p$ applied to a name $\mathrm{Y}$

$/ *$ For example: $\mathrm{X}=* \mathrm{p}, \mathrm{Y}=\mathrm{p}$, and $o p=* ; \quad * /$

/* The outermost find is needed because a partition*/

${ }^{*}$ is represented by a representative element. Since union can change*/

/* which element is the representative element, a find*/

$/ *$ is needed here. For simplicity, find(NULL) is NULL. */

if $\operatorname{find}(\mathrm{X}) \neq \operatorname{find}(\operatorname{map}[\operatorname{find}(\mathrm{Y})][o p])$

then

if $\operatorname{map}[\operatorname{find}(\mathrm{Y})][o p]$ is NULL

then redefine $\operatorname{map}[\operatorname{find}(\mathrm{Y})][o p]$ to be $\operatorname{find}(\mathrm{X})$

else $\operatorname{merge}(\operatorname{find}(\mathrm{X}), \operatorname{find}(\operatorname{map}[\operatorname{find}(\mathrm{Y})][o p]))$

/* Phase 3: Accounting for calls through function pointers*/

repeat

for each call through a function pointer which has arguments $\left(f p\left(\arg _{1}, \ldots, \arg _{n}\right)\right)$

for each function func in the partition of $\mathrm{fp}$ $\operatorname{merge}\left(\right.$ find $\left(* \arg _{i}\right)$,find $\left(*\right.$ form $\left.\left._{i}\right)\right)$ for all $1 \leq i \leq n$

where form $_{i}$ is the $i^{\text {th }}$ formal of func

until no changes in the partitions involving function pointers.

end

$/ *$ merge function used in Phases 2,3*/

function merge ( part $_{1}$, part $\left._{2}\right)$

begin if $\operatorname{find}\left(\right.$ part $\left._{1}\right) \neq \operatorname{find}\left(\right.$ part $\left._{2}\right)$

let $\operatorname{old}_{1}=\operatorname{map}\left[\right.$ find $\left.\left(\operatorname{part}_{1}\right)\right]$ and old $2=\operatorname{map}\left[\operatorname{find}\left(\operatorname{part}_{2}\right)\right]$

union $\left(\right.$ part $_{1}$, part $\left._{2}\right)$

$\operatorname{map}\left[\operatorname{find}\left(\operatorname{part}_{1}\right)\right]=\lambda x \cdot\left(\begin{array}{ll}\text { NULL } & \text { if } \operatorname{old}_{1}[x]=\operatorname{old}_{2}[x]=\text { NULL } \\ \operatorname{old}_{1}[x] & \text { if } \operatorname{old}_{2}[x]=\text { NULL } \\ \operatorname{old}_{2}[x] & \text { if old }[x]=\text { NULL } \\ \operatorname{merge}\left(\operatorname{old}_{1}[x], \operatorname{old}_{2}[x]\right) & \text { otherwise }\end{array}\right)$

end

return $\operatorname{find}\left(\operatorname{part}_{1}\right)$

Fig. 5. FIAlias algorithm.

Phase 3 is straightforward, accounting for calls through function pointers. fp can be an arbitrary object name; for example, $\operatorname{var}_{1}$, $* \operatorname{var}_{1}$, or $* * v^{2} r_{2}$. Care must be taken in $\mathrm{C}$ because the calls $\operatorname{var}(\ldots)$ and (*var) () are semantically equivalent and should be be treated as such. Because FSAlias as described does not handle function pointers, we did not implement Phase 3 of FIAlias. None of the $\mathrm{C}$ programs we used as data contained function pointers.

Figure 8 shows an example of Phases 1 and 2 of FIAlias as performed on the small $\mathrm{C}$ program given in Figure 7. Initially, each object name is placed in its own equivalence class; $(* * \mathrm{x}, * \mathrm{x}, \mathrm{x}, * \mathrm{y}, \mathrm{y}, \mathrm{a}, * \mathrm{a}, \mathrm{b})$ are all interesting names for this program. The object names $(* a, * y, b, * * x)$ do not appear themselves in 


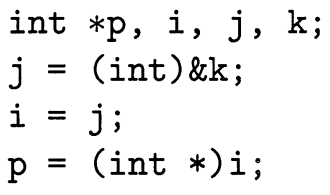

Fig. 6. Unsafe use of casting.

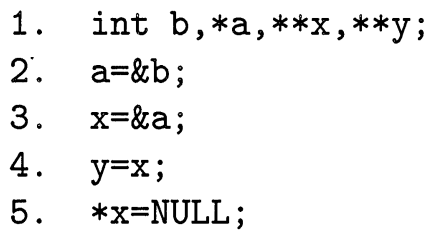

Fig. 7. A small C program.

executable statements, but are needed to calculate the aliases for this program. Note that $* * \mathrm{y}$ is not interesting, because neither $* * \mathrm{y}$ nor $* \mathrm{y}$ appear in the assignments in the program.

As shown, Phase I of FIAlias computes equivalence sets $\{* \mathrm{a}, \mathrm{b}\},\{* \mathrm{x}, \mathrm{a}, * \mathrm{y}\}$, $\{* * x\},\{x\},\{y\}$ by accounting for type 1 effects for each program statement. Phase II of FIAlias explores all the interesting names and essentially checks that if two interesting object names $\mathrm{w}, \mathrm{v}$ are in the same equivalence class and $* \mathrm{w}, * \mathrm{v}$ are also interesting, then $* \mathrm{w}, * \mathrm{v}$ are also in the same equivalence class. If this is not the case, the algorithm makes it so by merging the two corresponding equivalence classes; this is accomplished by using the map function. The first Update map step of Phase II effectively uses map to represent the points-to edge labeled $*$ going from equivalence class $\{\mathrm{x}\}$ to $\{* \mathrm{x}, \mathrm{a}, * \mathrm{y}\}$; this is analogous to drawing a labeled edge in a points-to graph between these two classes. Intermediate steps in the example effectively draw other edges labeled $*$ between appropriate equivalence classes. The last step of Phase II finds that $\{* * \mathrm{x}\}$ and $\{* a, b\}$ should belong to the same equivalence class because of type 2 effects with regard to $* \mathrm{x}$ and a, which are in the same equivalence class.

The final set of equivalence classes can be interpreted to mean that object names $* \mathrm{a}, * * \mathrm{x}$ share the same points-to set; namely, they both point to $\mathrm{b}$. Similarly, object names $* \mathrm{x}, * \mathrm{y}$ both point to a.

The operations in FIAlias are similar to those used by a unification-based flow-insensitive analysis [Steensgaard 1996b]; the solution calculated by both algorithms is the same. FIAlias can be shown to have worst case almost linear performance $O(N \alpha(N, N))$ where $N$ is bounded above by the number of object names appearing in the program [Zhang et al. 1996].

2.6.3 Example. Figure 9 demonstrates the differences in precision between FSAlias and FIAlias through a small example involving three procedures, main, proc1, and proc2. First, consider the solution obtained by FIAlias. Since $\mathrm{q}$ is assigned both the address of $\mathrm{a}$ and $\mathrm{b}$ in main, FIAlias will assume that the aliases $\langle * \mathrm{q}, \mathrm{a}\rangle$ and $\langle * \mathrm{q}, \mathrm{b}\rangle$ hold globally throughout the program. Because FSAlias propagates alias information through statements 7-14 in order, the alias $\langle * \mathrm{q}, \mathrm{a}\rangle$ holds on exit from statement 7 , through statements $8-9$, and is killed by statement 11; therefore, FSAlias can tell that b is the only fixed 


\begin{tabular}{|c|c|c|c|c|}
\hline Phase & Step & Action & Equiv Classes & Map \\
\hline & & Initialize & $\begin{array}{c}\mathrm{x}\}\{\mathrm{y}\}\left\{{ }^{*} \mathrm{x}\right\}\left\{{ }^{*} \mathrm{y}\right\}\{\mathrm{a}\} \\
\left\{{ }^{* *} \mathrm{x}\right\}\left\{{ }^{*} \mathrm{a}\right\}\{\mathrm{b}\}\end{array}$ & \\
\hline 1 & $\begin{array}{l}\text { Process } \\
\text { stmt } 2\end{array}$ & $\operatorname{Union}\left({ }^{*} \mathrm{a}, \mathrm{b}\right)$ & $\begin{array}{c}\{\mathrm{x}\}\{\mathrm{y}\}\left\{{ }^{*} \mathrm{x}\right\}\left\{{ }^{*} \mathrm{y}\right\}\{\mathrm{a}\} \\
\left\{{ }^{* *} \mathrm{x}\right\}\left\{{ }^{*} \mathrm{a}, \mathrm{b}\right\}\end{array}$ & \\
\hline 1 & $\begin{array}{l}\text { Process } \\
\text { stmt } 3\end{array}$ & $\operatorname{Union}\left({ }^{*} \mathrm{x}, \mathrm{a}\right)$ & $\begin{array}{c}\{\mathrm{x}\}\{\mathrm{y}\}\left\{{ }^{*} \mathrm{x}, \mathrm{a}\right\}\left\{{ }^{*} \mathrm{y}\right\} \\
\left\{{ }^{* *} \mathrm{x}\right\}\left\{{ }^{*} \mathrm{a}, \mathrm{b}\right\}\end{array}$ & \\
\hline 1 & $\begin{array}{l}\text { Process } \\
\text { stmt } 4\end{array}$ & $\operatorname{Union}\left({ }^{*} \mathrm{y},{ }^{*} \mathrm{x}\right)$ & $\begin{array}{c}\{\mathrm{x}\}\{\mathrm{y}\}\left\{{ }^{*} \mathrm{x}, \mathrm{a},{ }^{*} \mathrm{y}\right\} \\
\left\{*^{*} \mathrm{x}\right\}\left\{{ }^{*} \mathrm{a}, \mathrm{b}\right\}\end{array}$ & \\
\hline 1 & $\begin{array}{l}\text { Process } \\
\text { stmt } 5\end{array}$ & None & $\begin{array}{c}\{\mathrm{x}\}\{\mathrm{y}\}\left\{{ }^{*} \mathrm{x}, \mathrm{a},{ }^{*} \mathrm{y}\right\} \\
\{* * \mathrm{x}\}\left\{{ }^{*} \mathrm{a}, \mathrm{b}\right\}\end{array}$ & \\
\hline 2 & & Initialize & $\begin{array}{c}\{\mathrm{x}\}\{\mathrm{y}\}\left\{{ }^{*} \mathrm{x}, \mathrm{a},{ }^{*} \mathrm{y}\right\} \\
\left\{*^{*} \mathrm{x}\right\}\left\{{ }^{*} \mathrm{a}, \mathrm{b}\right\}\end{array}$ & $\begin{array}{l}\operatorname{map}[\{x\}]=\lambda x \cdot N U L L \\
\operatorname{map}[\{y\}]=\lambda x \cdot N U L L \\
\operatorname{map}\left[\left\{{ }^{*} x,{ }^{*} y, a\right\}\right]=\lambda x \cdot N U L L \\
\operatorname{map}[\{* * x\}]=\lambda x \cdot N U L L \\
\operatorname{map}[\{* a, b\}]=\lambda x \cdot N U L L\end{array}$ \\
\hline 2 & $\begin{array}{l}\mathrm{X}=* \mathrm{x}, \mathrm{Y}=\mathrm{x} \\
\mathrm{op}=*\end{array}$ & Update map & $\begin{array}{c}\{\mathrm{x}\}\{\mathrm{y}\}\left\{{ }^{*} \mathrm{x}, \mathrm{a},{ }^{*} \mathrm{y}\right\} \\
\left\{{ }^{* *} \mathrm{x}\right\}\left\{{ }^{*} \mathrm{a}, \mathrm{b}\right\}\end{array}$ & $\begin{array}{l}\text { Above except } \\
\operatorname{map}[\{x\}]\left[^{*}\right]=\left\{{ }^{*} x, a,{ }^{*} y\right\}\end{array}$ \\
\hline 2 & $\begin{array}{l}\mathrm{X}=* \mathrm{y}, \mathrm{Y}=\mathrm{y} \\
\mathrm{op}=*\end{array}$ & Update map & $\begin{array}{c}\{\mathrm{x}\}\{\mathrm{y}\}\left\{{ }^{*} \mathrm{x}, \mathrm{a},{ }^{*} \mathrm{y}\right\} \\
\{* * \mathrm{x}\}\left\{{ }^{*} \mathrm{a}, \mathrm{b}\right\}\end{array}$ & $\begin{array}{l}\text { Above except } \\
\operatorname{map}[\{\mathrm{y}\}]\left[^{*}\right]=\left\{{ }^{*} \mathrm{x}, \mathrm{a},{ }^{*} \mathrm{y}\right\}\end{array}$ \\
\hline 2 & $\begin{array}{l}\mathrm{X}={ }^{* *} \mathrm{x}, \mathrm{Y}={ }^{*} \mathrm{x} \\
\mathrm{op}=*\end{array}$ & Update map & $\begin{array}{c}\{\mathrm{x}\}\{\mathrm{y}\}\left\{{ }^{*} \mathrm{x}, \mathrm{a},{ }^{*} \mathrm{y}\right\} \\
\left\{{ }^{* *} \mathrm{x}\right\}\left\{{ }^{*} \mathrm{a}, \mathrm{b}\right\}\end{array}$ & $\begin{array}{l}\text { Above except } \\
\operatorname{map}\left[\left\{{ }^{*} \mathrm{x}, \mathrm{a},{ }^{*} \mathrm{y}\right\}\right][*]=\{* * \mathrm{x}\}\end{array}$ \\
\hline 2 & $\begin{array}{l}X={ }^{*} a, Y=a \\
o p=*\end{array}$ & $\operatorname{Union}\left(* * x,{ }^{*} \mathrm{a}\right)$ & $\begin{array}{c}\{\mathrm{x}\}\{\mathrm{y}\}\left\{{ }^{*} \mathrm{x}, \mathrm{a},{ }^{*} \mathrm{y}\right\} \\
\left\{{ }^{* *} \mathrm{x},{ }^{*} \mathrm{a}, \mathrm{b}\right\}\end{array}$ & $\begin{array}{l}\text { Implicitly replace } \\
\operatorname{map}\left[\left\{{ }^{*} \mathrm{a}, \mathrm{b}\right\}\right], \operatorname{map}\left[\left\{{ }^{*} \mathrm{x}\right\}\right] \text { by } \\
\operatorname{map}\left[\left\{*^{*} \mathrm{x},{ }^{*} \mathrm{a}, \mathrm{b}\right\}\right]=\lambda \mathrm{x} . \mathrm{NULL}\end{array}$ \\
\hline
\end{tabular}

Fig. 8. Example of FIAlias applied to program in Figure 7.

location aliased to $* \mathrm{q}$ at statement 12 , not both a and b which would be reported by FIAlias. Similarly, at statement 9, FSAlias can tell that only fixed location a is aliased to $*$ p whereas FIAlias cannot and reports $*$ p aliased to both $a$ and $b$.

To illustrate the interprocedural propagation of aliases by FSAlias, proc1 is called by main at statements 10 and 14 and proc2 is called at statement 15 . The alias tuple $[\phi,\langle * \mathrm{q}, \mathrm{a}\rangle]$ reaches the exit of statement 9 . This is propagated into proc1 as alias tuple $[\langle * \mathrm{q}, \mathrm{a}\rangle,\langle * \mathrm{q}, \mathrm{a}\rangle]$ and reaches the exit of statement 3 . Then, alias tuple $[\langle * \mathrm{q}, \mathrm{a}\rangle,\langle * \mathrm{r}, \mathrm{a}\rangle]$ is created on exit of statement 4 , and it and the alias tuple $[\langle * \mathrm{q}, \mathrm{a}\rangle,\langle * \mathrm{q}, \mathrm{a}\rangle]$ reach the exit of statement 5 . Finally, the alias tuples $([\phi,\langle * \mathrm{q}, \mathrm{a}\rangle],[\phi,\langle * \mathrm{r}, \mathrm{a}\rangle])$ reach the exit of the call statement 10.

Statement 11 then kills alias tuple $[\phi,\langle * \mathrm{q}, \mathrm{a}\rangle]$, and creates the alias tuple $[\phi,\langle * \mathrm{q}, \mathrm{b}\rangle]$ which reaches the call at statement 14 . Alias tuple $[\phi,\langle * \mathrm{r}, \mathrm{a}\rangle]$ is preserved through statements $11-13$ and reaches the call at statement 14. Alias tuple $[\phi,\langle * r, a\rangle]$ will result in $[\langle * r, a\rangle,\langle * r, a\rangle]$ being propagated to the exit of statement 3 . Then this is killed by statement 4 . Alias tuple $[\phi,\langle * \mathrm{q}, \mathrm{b}\rangle]$ will result in $[\langle * \mathrm{q}, \mathrm{b}\rangle,\langle * \mathrm{q}, \mathrm{b}\rangle]$ being propagated to the exit of statement 3 . This results in alias tuple $[\langle * \mathrm{q}, \mathrm{b}\rangle,\langle * \mathrm{r}, \mathrm{b}\rangle]$ at the exit of statement 4 , which reaches the exit of statement 5 as does $[\langle * \mathrm{q}, \mathrm{b}\rangle,\langle * \mathrm{q}, \mathrm{b}\rangle]$. Finally, alias tuples $([\phi,\langle * \mathrm{q}, \mathrm{b}\rangle]$, $[\phi,\langle * \mathrm{r}, \mathrm{b}\rangle])$ reach the exit of the call statement 14 , and thus the entry of call statement 15. Similarly, these tuples, $[\phi,\langle * \mathrm{q}, \mathrm{b}\rangle]$ and $[\phi,\langle * \mathrm{r}, \mathrm{b}\rangle]$, cause alias tuples $[\langle * f, a\rangle,\langle * \mathrm{q}, \mathrm{b}\rangle]$ and $[\langle * \mathrm{f}, \mathrm{a}\rangle,\langle * \mathrm{r}, \mathrm{b}\rangle]$ to reach statement 16 . In addition, tuple $[\langle * \mathrm{f}, \mathrm{a}\rangle,\langle * \mathrm{f}, \mathrm{a}\rangle]$ reaches statement 16 because procedure proc 2 has a formal 


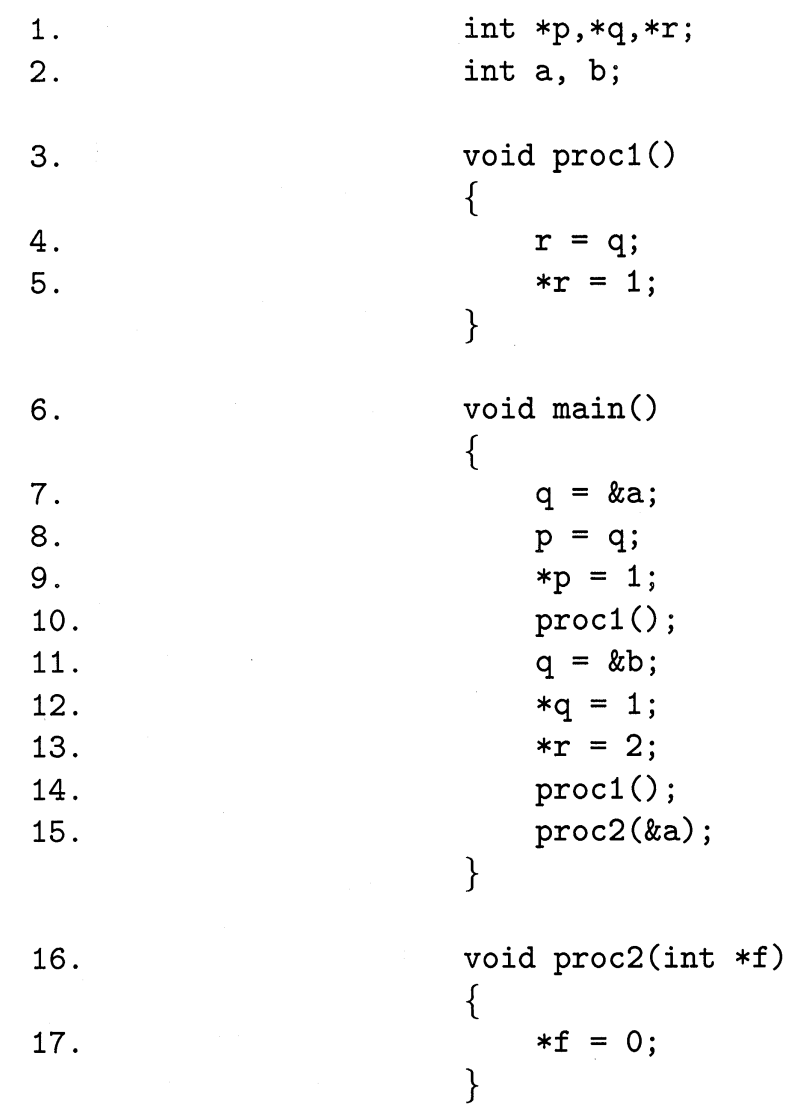

Fig. 9. Example of differences between FIAlias and FSAlias.

parameter and parameter association is treated as an implicit assignment (i.e., 1.2 alias_intro_by_call()). All of these tuples are preserved through statement 17, and no new tuple is created. On return from procedure proc2, tuples $[\phi,\langle * \mathrm{q}, \mathrm{b}\rangle]$ and $[\phi,\langle * r, b\rangle]$ are passed back to the exit of statement 15.

Although alias tuples $[\langle * \mathrm{q}, \mathrm{a}\rangle,\langle * r, \mathrm{a}\rangle]$ and $[\langle * \mathrm{q}, \mathrm{b}\rangle,\langle * \mathrm{r}, \mathrm{b}\rangle]$ both reach the exit of statement 5 in proc1, the reaching alias abstraction ensures that these aliases are mapped back only to the corresponding calling contexts; therefore, alias tuple $[\phi,\langle * r, a\rangle]$ will be mapped back to the exit of statement 10 , and alias tuple $[\phi,\langle * r, b\rangle]$ will be mapped back to the exit of statement 14 . Thus, the only fixed location aliased to $* r$ at statement 13 is a with FSAlias, but FIAlias will find both a and b aliased to $* \mathrm{r}$, since it combines all calling contexts. Note that $* \mathrm{r}$ is reported aliased to both $\mathrm{a}$ and $\mathrm{b}$ at statement 5 using either alias algorithm.

\section{3. $\mathrm{MOD}_{C}$ SCHEMA}

The $\mathrm{MOD}_{C}$ schema defines a family of algorithms which solve for modification side effects to fixed locations at program points, parameterized by the type of aliasing algorithm used. Side effects reported are differentiated by fixedlocation type: global, local, dynamically-created, and non-visible. In solving for 


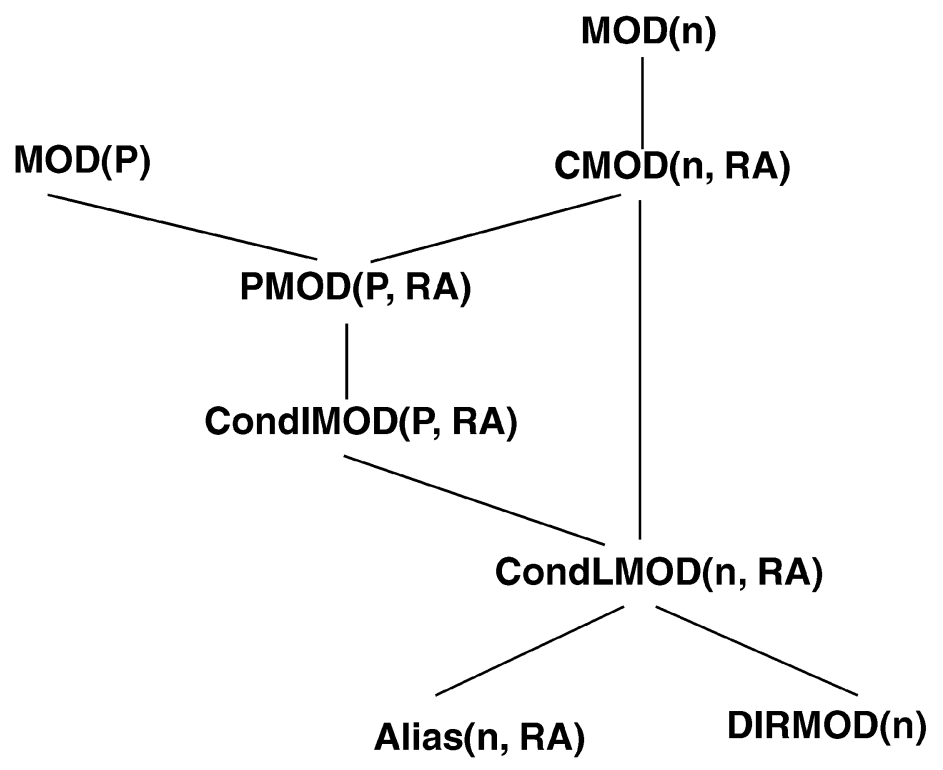

Fig. 10. Decomposition of the $\mathrm{MOD}_{C}$ problem.

modification side effects, the $\mathrm{MOD}_{C}$ problem is decomposed into subproblems that are individually easier to solve than the monolithic problem. The problem decomposition assumes that context-sensitive alias information is available; it preserves calling-context information with the side effects for as long as possible. The $\mathrm{MOD}_{C}$ schema is described for FSAlias, but any approximation of calling context may be used instead. If the pointer alias algorithm used is context-insensitive, then conceptually all calling contexts are mapped to one context; that is, there is no differentiation in side effects returned from a procedure to any individual call site, and the multiple subproblems distinguished by $R A$ shown in Figure 10 become a single subproblem.

As mentioned previously, the first pass of the algorithm solves for aliasing information, Alias. Given the results of this analysis, two related problems are calculated: (i) PMOD, a procedure-level summary of context-sensitive modification side effects which can occur to fixed locations, and (ii) $C M O D$, a set of modified fixed locations at each program point corresponding to a specific context. CMOD solutions can then be used to derive MOD information for program points, while $P M O D$ solutions are a procedure-level summary of modification side effects.

The decomposition of the MOD problem is pictured in Figure 10, where $P$ is a procedure, $R A$ is a calling context (i.e., a reaching alias), and $n$ is a program point. The following brief description of each subproblem will be augmented in the next section by the corresponding data-flow equations. Alias $(n, R A)$ is the pointer-alias solution at statement $n$ under calling context $R A . D I R M O D(n)$ captures all object names which occur on the left-hand-side of the assignment at program point $n$ (e.g., $* \mathrm{p}=, \mathrm{v}=$ ). At an assignment $n$, CondLMOD widens $\operatorname{DIRMOD}(n)$ to include the effects of aliasing; CondLMOD contains only fixed 
locations. CondIMOD $(P, R A)$ summarizes CondLMOD information for each calling context $R A$ over all assignment statements in procedure $P$. $P M O D(P$, $R A$ ) is formed from local CondIMOD information for $P$ and $P M O D$ information propagated from procedures called by $P$ all under context $R A$, thus calculating both direct and indirect side effects of $P$. CMOD at a call site is constructed from $P M O D$ of the called procedure, and at an assignment, from CondLMOD of that statement. Finally, $M O D$ at a statement is constructed from $C M O D$ by summarizing over all contexts, as is $M O D$ for a procedure.

A comparison of our $\mathrm{MOD}_{C}$ decomposition to that for FORTRAN is given in Appendix A. Recall, that although the $\mathrm{MOD}_{C}$ schema is described with calling-context information available, an easy transformation (i.e., folding all contexts together) yields a $\mathrm{MOD}_{C}$ algorithm for use with context-insensitive alias methods.

\subsection{Data-Flow Equations}

The following discussion makes these assumptions.

- Assignment is synonymous with value-setting statement; thus, scanf is considered an assignment.

- All object names are unique; thus the issue of name hiding is avoided. This can easily be met by appending object names with the function and file in which they are defined.

- On bottom data-flow information is computed (i.e., information at a statement incorporates the effects of that statement). Since a call statement is split into a call node and a return node in the internal representation, the information computed at the return node is "on bottom" information for the call statement, while the information computed at the call node is "on top" information for the call statement.

- The modification side-effects sets are associated with some representation of calling context to restrict attention to realizable paths; note that the $\mathrm{MOD}_{C}$ schema is independent of the choice of calling-context abstraction.

- Predecessors $(n)$ represents the set of predecessors of $n$ in the ICFG.

- Trivial, reflexive aliases (e.g., $\langle * \mathrm{p}, * \mathrm{p}\rangle)$ are associated with the special reaching alias $\phi$ at all program points; this assumption simplifies the equation for CondLMOD. In the actual implementation these trivial aliases are not stored.

$\operatorname{DIRMOD}(n)$ is defined as the visible direct side effects at a statement; therefore, it requires no data-flow equation. $\operatorname{CondLMOD}(n, R A)$ is the set of fixed locations modified by the assignment at $n$ because of aliases that occur on bottom of any of the predecessors of $n$ under calling context $R A$ for the procedure containing $n$ :

$$
\operatorname{CondLMOD}(n, R A)=\bigcup_{\text {pred } \in \text { Predecessors }(n)}\left\{\begin{array}{l|l}
o b j_{1} & \begin{array}{l}
o b j_{2}=\operatorname{DIRMOD}(n) \text { and } \\
\left\langle o b j_{1}, o b j_{2}\right\rangle \in \operatorname{Alias}(\operatorname{pred}, R A) \\
\text { and } o b j_{1} \text { is a fixed location }
\end{array}
\end{array}\right\}
$$


If $\operatorname{DIRMOD}(n)$ is a fixed location, it is included in $\operatorname{CondLMOD}(n, \phi)$ because reflexive aliases are associated with the special reaching alias $\phi$.

For a procedure $P$ and each calling context $R A, \operatorname{CondIMOD}(P, R A)$ contains the fixed locations modified by assignments in procedure $P$.

$$
\operatorname{CondIMOD}(P, R A)=\bigcup_{n \text { an assignment in } P} \operatorname{CondLMOD}(n, R A)
$$

$\operatorname{PMOD}(P, R A)$ is the set of fixed locations modified by procedure $P$, including the effects of calls from within $P$, considering only aliases corresponding to calling context $R A$. The $P M O D$ sets for a procedure summarize its modification side effects for a given reaching alias. They are specified by the following, possibly recursive, system of data-flow equations which can be solved iteratively. The fixed-point iteration used in the implementation is an optimistic lattice framework, ${ }^{11}$ which has been highly optimized with respect to the interprocedural transfer functions.

$$
P M O D(P, R A)=\operatorname{CondIMOD}(P, R A) \cup \underset{\begin{array}{c}
\text { call }_{Q} \text { in } P \text { and } \\
R A^{\prime} \in \text { contexts_of }\left(\text { call }_{Q}, R A\right)
\end{array}}{\bigcup}\left(b_{\text {call }_{Q}}\left(P M O D\left(Q, R A^{\prime}\right)\right)\right)
$$

In Equation (3), call $_{Q}$ is a call site in $P$ at which $P$ calls $Q . R A^{\prime}$ represents the calling context induced by call $_{Q}$ during data-flow propagation in $P$ under calling context $R A$ of $P$. The function $b_{\text {call }_{Q}}$, specific to $\operatorname{call}_{Q}$, maps object names from the called procedure $(Q)$ to the calling procedure $(P)$ according to scoping rules [Cooper and Kennedy 1987] and only returns fixed locations. Specifically, $b_{\text {call }_{Q}}$ factors out all local fixed locations of $Q$ (including formal parameters of $Q$ ), maps global fixed locations (including heap storage creation sites) to themselves, and maps non-visibles in $Q$ to their corresponding fixed locations in $P$, which are either locals of $P$ or non-visibles in $P$ [Landi and Ryder 1992]. ${ }^{12}$ Recall that contexts_of are sets of reaching aliases defined in Section 2.6.1.

It is possible with the $\mathrm{MOD}_{C}$ schema to derive side effects at specific interesting statements, namely calls and assignments.

$$
\operatorname{CMOD}(n, R A)= \begin{cases}\operatorname{CondLMOD}(n, R A) & \text { if } n \text { is an assignment } \\ \bigcup_{R A^{\prime} \in \text { contexts_of }(n, R A)} b_{n}\left(\operatorname{PMOD}\left(\mathrm{Q}, R A^{\prime}\right)\right) & \text { if } n \text { is a call of } \mathrm{Q} \\ \emptyset & \text { otherwise }\end{cases}
$$

Finally, $M O D(n)$ summarizes the side effects over all executions of $n$ in procedure $P$, and $M O D(P)$ summarizes the side effects over all calls of $P$. Both are obtained by considering all contexts for $P$.

\footnotetext{
${ }^{11}$ An optimistic data-flow framework requires iteration to a fixed point to obtain a safe solution; by contrast, partial solutions of a pessimistic framework are safe.

${ }^{12}$ If context-insensitive aliases are used, there are no non-visibles. 

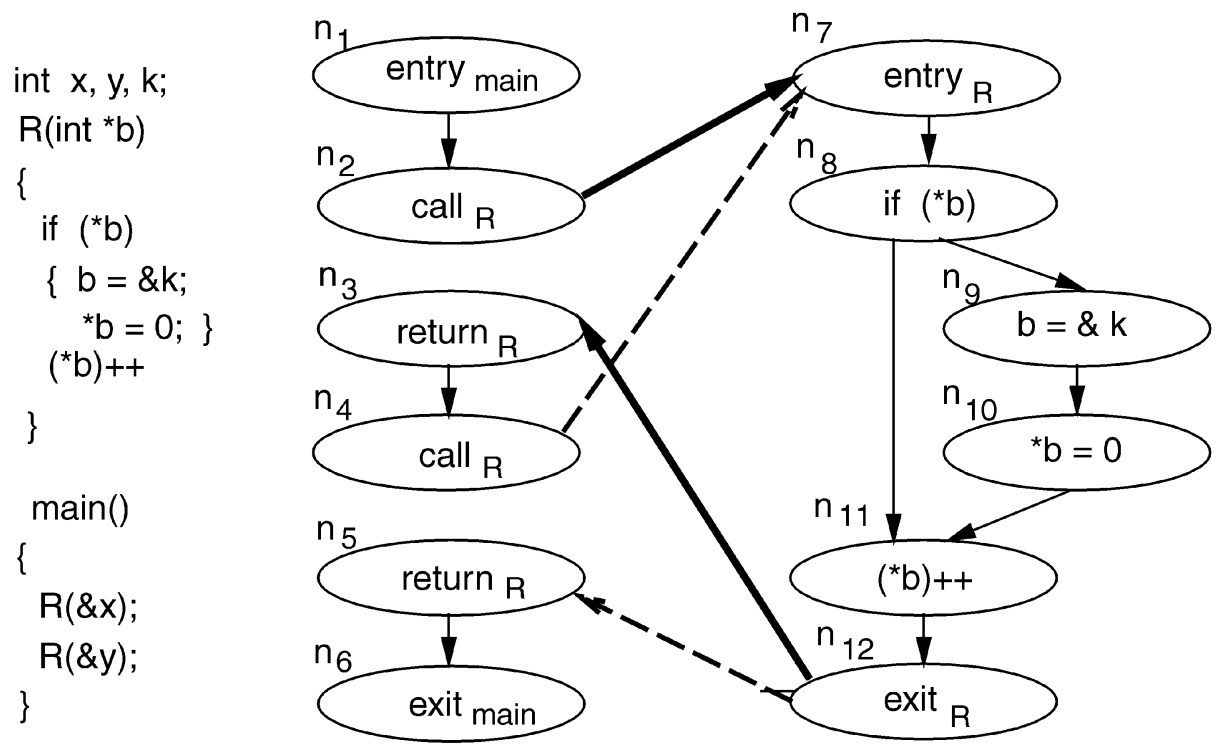

Fig. 11. An example program and its ICFG.

$$
\begin{aligned}
& M O D(n)=\bigcup_{\text {context } R A \text { for } P} \operatorname{CMOD}(n, R A) \\
& M O D(P)=\bigcup_{\text {context } R A \text { for } P} \operatorname{PMOD}(P, R A)
\end{aligned}
$$

\subsection{Example}

The example in Figures 11, 12, and 13 shows a small $\mathrm{C}$ program analyzed by both the $\operatorname{MOD}_{C}($ FSAlias $)$ and $\operatorname{MOD}_{C}($ FIAlias $)$ algorithms.

For $\operatorname{MOD}_{C}($ FSAlias $)$, both main and $\mathrm{R}$ are analyzed with reaching alias $\phi$ at their entries. The first call to $\mathrm{R}$ (shown by the solid line) creates the alias $\langle * \mathrm{~b}, \mathrm{x}\rangle$ at the entry of $\mathrm{R}$. The second call to $\mathrm{R}$ (shown by the dashed line) creates the alias $\langle * \mathrm{~b}, \mathrm{y}\rangle$ at the entry of $\mathrm{R}$. Procedure $\mathrm{R}$ is analyzed for each of these calling contexts. Note there are no aliases in main. The FSAlias solution for $\mathrm{R}$ is shown in Figure 12; the PMOD and CMOD solutions computed are shown in the same figure. Empty entries in these tables mean either no alias or no side effect. Note that the entries in the tables indicate additions to the solution at a program point under a calling context. The whole solution at a point under a given context is the union of the entry in the table and the solution under calling context $\phi$. Fixed location $\mathrm{b}$ is not in the solution for main because it is a local of $R$.

For $\mathrm{MOD}_{C}($ FIAlias $)$, the FIAlias solution is shown in Figure 13. The PMOD solutions for main and $\mathrm{R}$ are shown as well. The $C M O D$ solution does differentiate side effects at program points within main and $\mathrm{R}$, but notice that calling contexts are not differentiated. 


\begin{tabular}{|c||c|c|c|c|c|c|}
\hline \multicolumn{1}{|c||}{$\begin{array}{c}\text { Reaching } \\
\text { Alias }\end{array}$} & \multicolumn{7}{c|}{ Alias Solutions for $\mathrm{R}$} \\
\cline { 2 - 7 } & $n_{7}$ & $n_{8}$ & $n_{9}$ & $n_{10}$ & $n_{11}$ & $n_{12}$ \\
\hline \hline$\phi$ & & & $<* \mathrm{~b}, \mathrm{k}>$ & $<* \mathrm{~b}, \mathrm{k}>$ & $<* \mathrm{~b}, \mathrm{k}>$ & $<* \mathrm{~b}, \mathrm{k}>$ \\
\hline$<* \mathrm{~b}, \mathrm{x}>$ & $<* \mathrm{~b}, \mathrm{x}>$ & $<* \mathrm{~b}, \mathrm{x}>$ & & & $<* \mathrm{~b}, \mathrm{x}>$ & $<* \mathrm{~b}, \mathrm{x}>$ \\
\hline$<* \mathrm{~b}, \mathrm{y}>$ & $<* \mathrm{~b}, \mathrm{y}>$ & $<* \mathrm{~b}, \mathrm{y}>$ & & & $<* \mathrm{~b}, \mathrm{y}>$ & $<* \mathrm{~b}, \mathrm{y}\rangle$ \\
\hline
\end{tabular}

\begin{tabular}{|c||c|}
\hline Reaching Alias & $P M O D$ Solutions for main \\
\hline \hline$\phi$ & $\{\mathrm{x}, \mathrm{k}, \mathrm{y}\}$ \\
\hline
\end{tabular}

\begin{tabular}{|c||c|}
\hline Reaching Alias & $P M O D$ Solutions for $\mathrm{R}$ \\
\hline \hline$\phi$ & $\{\mathrm{k}, \mathrm{b}\}$ \\
\hline$<* \mathrm{~b}, \mathrm{x}>$ & $\{\mathrm{x}\}$ \\
\hline$<* \mathrm{~b}, \mathrm{y}>$ & $\{\mathrm{y}\}$ \\
\hline
\end{tabular}

\begin{tabular}{|c|c|c|c|c|c|c|c|c|c|c|c|c|c|}
\hline \multirow{3}{*}{$\begin{array}{c}\text { Reaching } \\
\text { Alias }\end{array}$} & & & & & & & \multirow{2}{*}{$\begin{array}{c}\text { Reaching } \\
\text { Alias }\end{array}$} & \multicolumn{6}{|c|}{$C M O D$, Solutions for $\mathrm{R}$} \\
\hline & \multicolumn{6}{|c|}{$C M O D$ Solutions for main } & & $n_{7}$ & $n_{8}$ & $n_{9}$ & $n_{10}$ & $n_{11}$ & $n_{12}$ \\
\hline & $n_{1}$ & $n_{2}$ & $n_{3}$ & $n_{4}$ & $n_{5}$ & $n_{6}$ & $\overline{\phi \phi}$ & & & $\{\mathrm{b}\}$ & $\{\mathrm{k}\}$ & $\{\mathrm{k}$ & \\
\hline$\phi$ & & $\{x, k\}$ & & $\{\mathrm{y}, \mathrm{k}\}$ & & & $<* \mathrm{~b}, \mathrm{x}\rangle$ & & & & & $x$ & \\
\hline & & & & & & & $<* \mathrm{~b}, \mathrm{y}\rangle$ & & & & & $\mathrm{y}$ & \\
\hline
\end{tabular}

Fig. 12. $\operatorname{MOD}_{C}($ FSAlias $)$ solution for the example program in Figure 11.

\subsection{Handling Call-by-Reference Parameters}

If a language uses call-by-reference parameter passing rather than the call-byvalue parameter passing as in $\mathrm{C}$, programs can be transformed into equivalent call-by-value programs by adding an additional level of indirection using the following transformation:

- For every call-by-reference formal $r$ of type $t_{r}$

1. make $r$ a call-by-value formal of type $t_{r} *$

2. replace $r$ everywhere in the procedure with $(* r)$

- For every actual a corresponding to a call-by-reference formal

3. replace a with \& (a); note that $a$ is an arbitrary object name (e.g., a [10], p->next)

When 2 and 3 above are both applicable, apply both. This means that a reference formal $r^{\prime}$ passed as an actual to another reference formal is transformed into $\&\left(* r^{\prime}\right)$. Since semantically, dereference $(*)$ and address $(\&)$ are effectively inverse operations ${ }^{13}\left(\& * r^{\prime} \equiv * \& r^{\prime} \equiv r^{\prime}\right)$, in the above case $r^{\prime}$ will be unchanged. This transformation is essentially the inverse of refizing as developed in Carroll [1988]; for programs with single-level pointers it is equivalent to the transformation in Landi [1992a, p. 50].

\subsection{Worst-Case Complexity}

The following definitions are useful in arguing the worst-case complexity of the $\mathrm{MOD}_{C}$ schema.

\footnotetext{
${ }^{13}$ In $\mathrm{C}$, there is an exception: $\& *(\& \mathrm{x}) \equiv \& \mathrm{x}$ but $* \&(\& \mathrm{x})$ is illegal. The transformation will never cause this kind of translation.
}

ACM Transactions on Programming Languages and Systems, Vol. 23, No. 1, March 2001. 


\begin{tabular}{|c|}
\hline FI Alias solution for entire program \\
\hline \hline$<* \mathrm{~b}, \mathrm{k}>$ \\
\hline$<* \mathrm{~b}, \mathrm{x}>$ \\
\hline$<* \mathrm{~b}, \mathrm{y}>$ \\
\hline
\end{tabular}

\begin{tabular}{|c|c|}
\hline PMOD Solution for main & PMOD Solution for $\mathrm{R}$ \\
\hline \hline$\{\mathrm{x}, \mathrm{y}, \mathrm{k}\}$ & $\{\mathrm{x}, \mathrm{y}, \mathrm{k}, \mathrm{b}\}$ \\
\hline
\end{tabular}

\begin{tabular}{|c|c|c|c|c|c||c|c|c|c|c|c|}
\hline \multicolumn{1}{|c|}{ CMOD Solutions for main } & \multicolumn{1}{c|}{ CMOD Solutions for R } \\
\hline \hline$n_{1}$ & $n_{2}$ & $n_{3}$ & $n_{4}$ & $n_{5}$ & $n_{6}$ & $n_{7}$ & $n_{8}$ & $n_{9}$ & $n_{10}$ & $n_{11}$ & $n_{12}$ \\
\hline & $\{\mathrm{k}, \mathrm{x}, \mathrm{y}\}$ & & $\{\mathrm{k}, \mathrm{x}, \mathrm{y}\}$ & & & & & $\{\mathrm{b}\}$ & $\{\mathrm{k}, \mathrm{x}, \mathrm{y}\}$ & $\{\mathrm{k}, \mathrm{x}, \mathrm{y}\}$ & \\
\hline
\end{tabular}

Fig. 13. $\operatorname{MOD}_{C}($ FIAlias $)$ solution for the example program of Figure 11.

- $N_{\text {alias }}$ is the total number of aliases in the program.

- $N_{\text {assign }}$ is the number of assignments in the program.

- $N_{\text {fixed }}$ is the number of fixed locations.

- $N_{I C F G}$ is the number of nodes in the ICFG. This is roughly equivalent to number of program points.

- $N_{\text {proc }}$ is the number of procedures in the program.

- $C_{\text {copy }}$ is the cost of copying a set of fixed locations $\left[\Omega\left(N_{\text {fixed }}\right)\right]$.

- $C_{\text {union }}$ is the cost of the union operation over sets of fixed locations $\left[\Omega\left(N_{\text {fixed }}\right)\right]$.

- $E_{I C F G}$ is the number of edges in the ICFG.

- $M_{\text {call }}$ is the maximum number of calls for any one procedure.

- $M_{\text {pred }}$ is the maximum number of predecessors of any assignment.

- $M_{R A}$ is the maximum number of reaching aliases at the entry of any procedure.

The worst-case time complexity of a $\mathrm{MOD}_{C}$ calculation is

$$
\begin{aligned}
& \mathcal{O}\left(N_{\text {proc }} * M_{\text {call }} * M_{R A}^{2} * N_{\text {fixed }}^{2}+N_{\text {assign }} * M_{R A} * M_{\text {pred }} * C_{\text {union }}\right. \\
& \left.\quad+N_{\text {alias }}+N_{I C F G} * M_{R A} * C_{\text {union }}\right)
\end{aligned}
$$

Nevertheless, as for most static analyses, the worst-case time has little correlation with the observed behavior of the algorithm in practice.

To understand the sources of complexity in an algorithm, examine worst-case time complexity of each calculation in turn.

- DIRMOD: $\mathcal{O}\left(N_{I C F G}\right)$

- Predecessors: $\mathcal{O}\left(E_{I C F G}\right)$

- CondLMOD: $\mathcal{O}\left(N_{\text {alias }}+N_{\text {assign }} * M_{R A} * M_{\text {pred }} * C_{\text {union }}\right)$

Access to the alias solution is necessary to compute CondLMOD, but each alias need only be considered once $\left[\mathcal{O}\left(N_{\text {alias }}\right)\right]$. There are at most $\left(N_{\text {assign }} * M_{R A}\right)$ CondLMOD sets, and for each one at most $\left(M_{\text {pred }}+1\right)$ unions are performed.

- CondIMOD: $\mathcal{O}\left(N_{\text {assign }} * M_{R A} * C_{\text {union }}\right)$

Each $\operatorname{CondLMOD}(n, R A)$, with $n$ an assignment statement and $R A$ a reaching alias, is conjoined into exactly one CondIMOD. Thus at most $\left(N_{a s s i g n} * M_{R A}\right)$ unions are performed. 
- PMOD: $\mathcal{O}\left(N_{\text {proc }} * M_{\text {call }} * M_{R A}^{2} * N_{\text {fixed }}^{2}\right)$

$P M O D$ requires a fixed-point calculation. There are at most $\left(N_{\text {proc }} * M_{R A}\right)$ $P M O D$ sets. $P M O D(P, R A)$ is first initialized to $\operatorname{CondIMOD}(P, R A)$. For all procedures $P$ and reaching aliases $R A$, this costs

$$
\mathcal{O}\left(N_{\text {proc }} * M_{R A} * C_{\text {copy }}\right) .
$$

Secondly, $P M O D$ is computed once for all procedures $P$ and contexts $R A$ using Eq. (3); this cost will be amortized over all calls in the program. There are $\mathcal{O}\left(N_{\text {proc }} * M_{\text {call }}\right)$ calls. For a call to procedure $Q$, each set $P M O D\left(Q, R A^{\prime}\right)$ will be considered at most $M_{R A}$ times per union operation. There are at most $M_{R A}$ such $P M O D$ sets for $Q$. Therefore the cost of unions at a call is at most $\left(M_{R A} * M_{R A}\right) *\left(N_{\text {fixed }}+C_{\text {union }}\right)$. The second term is the cost of the union as well as applying $b_{\text {call }}$ to each element. The cost of contexts_of and $b_{\text {call }_{Q}}$ is negligible as these functions are already calculated by the alias calculation, and for the second pass are implemented as a simple (hash) table lookup. Including the cost of a union with CondIMOD in Eq. (3), the total cost of computing $P M O D$ once is

$$
\begin{aligned}
& \mathcal{O}([\text { number of calls }] * \text { [cost of unions per call }] \\
& \quad+[\text { number of } P M O D \mathrm{~s}] * \text { [cost of one union }])
\end{aligned}
$$

Taking into account that $C_{\text {union }}=\Omega\left(N_{\text {fixed }}\right)$ and the first term dominates, this is equal to

$$
\mathcal{O}\left(\left[N_{\text {proc }} * M_{\text {call }}\right] *\left[M_{R A} * M_{R A} * N_{\text {fixed }}\right]\right) .
$$

Finally the cost of the iteration must be counted. Each $P M O D$ can change its value at most $N_{\text {fixed }}$ times. Thus there are at most $N_{\text {proc }} * M_{R A} * N_{\text {fixed }}$ changes over all $P M O D$ s. When $P M O D\left(Q, R A^{\prime}\right)$ changes, $P M O D(P, R A)$ of all

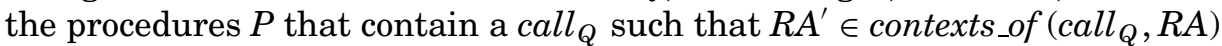
must be recomputed. There are at most $M_{\text {call }} * M_{R A}$ such $P M O D$ s. Thus, the cost of changing $P M O D(P, R A)$ is $\mathcal{O}\left(C_{\text {union }}+N_{\text {fixed }}\right)$ : one union (we do not recompute Eq. (3) from scratch) plus the cost of applying $b_{\text {call }}$ to each element of $\operatorname{PMOD}\left(Q, R A^{\prime}\right)$. The cost of the fixed-point iteration phase is $\mathcal{O}$ ([number of changes $] *$ [recomputations per change $] *[$ cost of one recomputation $])=$

$$
\begin{aligned}
& \mathcal{O}\left(\left[N_{\text {proc }} * M_{R A} * N_{\text {fixed }}\right] *\left[M_{\text {call }} * M_{R A}\right] * N_{\text {fixed }}\right) \\
& \quad=\mathcal{O}\left(N_{\text {proc }} * M_{\text {call }} * M_{R A}^{2} * N_{\text {fixed }}^{2}\right) .
\end{aligned}
$$

Thus, the total cost for computing PMOD is the sum of Eqs. (5), (6), and (7), which is $\mathcal{O}\left(N_{\text {proc }} * M_{\text {call }} * M_{R A}^{2} * N_{\text {fixed }}^{2}\right)$.

- CMOD: $\mathcal{O}\left(N_{\text {assign }} * M_{R A} * C_{\text {copy }}+N_{\text {proc }} * M_{\text {call }} * M_{R A}^{2} * N_{\text {fixed }}\right)$

For each assignment statement and each reaching alias, the cost is $C_{\text {copy }}$. For each call, call $P$, (there are $\mathcal{O}\left(N_{\text {proc }} * M_{\text {call }}\right)$ of them in the program) and each reaching alias, $R A, b_{\text {call }_{P}}$ is applied to $\operatorname{PMOD}(P, R A)$, and the results are conjoined. Thus, the cost for each $\left(\operatorname{call}_{P}, R A\right)$ pair is $\mathcal{O}\left(M_{R A} * N_{\text {fixed }}\right)$.

- $M O D: \mathcal{O}\left(N_{I C F G} * M_{R A} * C_{\text {union }}\right)$ 


\subsection{Counting Side Effects}

An important issue in measuring the effectiveness of a data-flow analysis is the choice of an empirically observable metric by which to judge performance. The number of fixed locations reported experiencing side effects at an assignment, at a call, and for a procedure is the metric used in these experiments. This seems reasonable, since it is of clear use in program understanding and compiling applications; if the number of measured side effects is too large at a program point, the analysis is not of practical use.

Some assignment statements in $\mathrm{C}$ involve aggregate types such as structs or unions. An aggregate is a fixed location whose fields can be simultaneously modified through one assignment. Arrays in $\mathrm{C}$ are not aggregates, because an array itself cannot be modified as one entity; all modifications occur through individual array elements.

Aggregates in $\mathrm{MOD}_{C}$ solutions present problems in counting the numbers of fixed locations modified.

Suppose $\mathrm{s}$ is a struct type with fields a, b, and c. It is possible that s, s.a, s.b, and s.c all are modified by individual assignment statements, and therefore all are found in a $\mathrm{MOD}_{C}$ solution for a particular procedure. For example, in main in Figure 14 there are assignments to every field of struct s3. In procedure $\mathrm{p}$, there is a struct assignment which simultaneously assigns to all three fields of struct $\mathrm{s} 1$. If struct fields are counted as fixed locations, then 3 side effects will be reported for main (one for each assignment); otherwise, 1 side effect to struct $\mathrm{s} 3$ will be reported. Similar questions determine if 1 or 3 fixed locations will be reported as side effects for procedure $p$ or if 1 or 3 fixed locations will be reported for procedure $r$. Note that the difference between $q$ and $r$ is that $r$ has a struct assignment and a field assignment to the same struct, whereas q has a struct assignment and a field assignment to a different struct.

The $\mathrm{MOD}_{C}$ implementation supports two counting schemes, called Fields and NoFields, respectively. To explain how aggregates are handled in the two counting schemes, refer to Figure 14. First an appropriate $\mathrm{MOD}_{C}$ algorithm is applied to a program and sets of fixed locations collected at each assignment statement. For procedures like $r$ both the entire struct and an individual field may occur in $\operatorname{MOD}(r)$ as a fixed-location side effect.

Counting fixed locations modified for an assignment statement is straightforward. For NoFields counting, any field name or struct name counts as 1 fixed location. For Fields counting, a struct assignment statement affects $m$ fixed locations for a struct with $m$ fields; the effect would be the same for an indirect access to the entire struct through a pointer to it. Each assignment to one field of a struct counts as 1 fixed location as well. These counts are illustrated in the Fields column in the code in Figure 14 for procedure q.

Counting becomes more complicated at a call when both a struct name and one of its field names are reported; this corresponds to the call of $r$ in main in Figure 14. Intuitively, if Fields counting is used, each distinct field is counted separately; thus the struct assignment statement in $r$ finds 3 fixed locations experiencing side effects whereas the assignment to s3. a finds only 1 . If NoFields 
Number of Fixed Locations Reported Modified

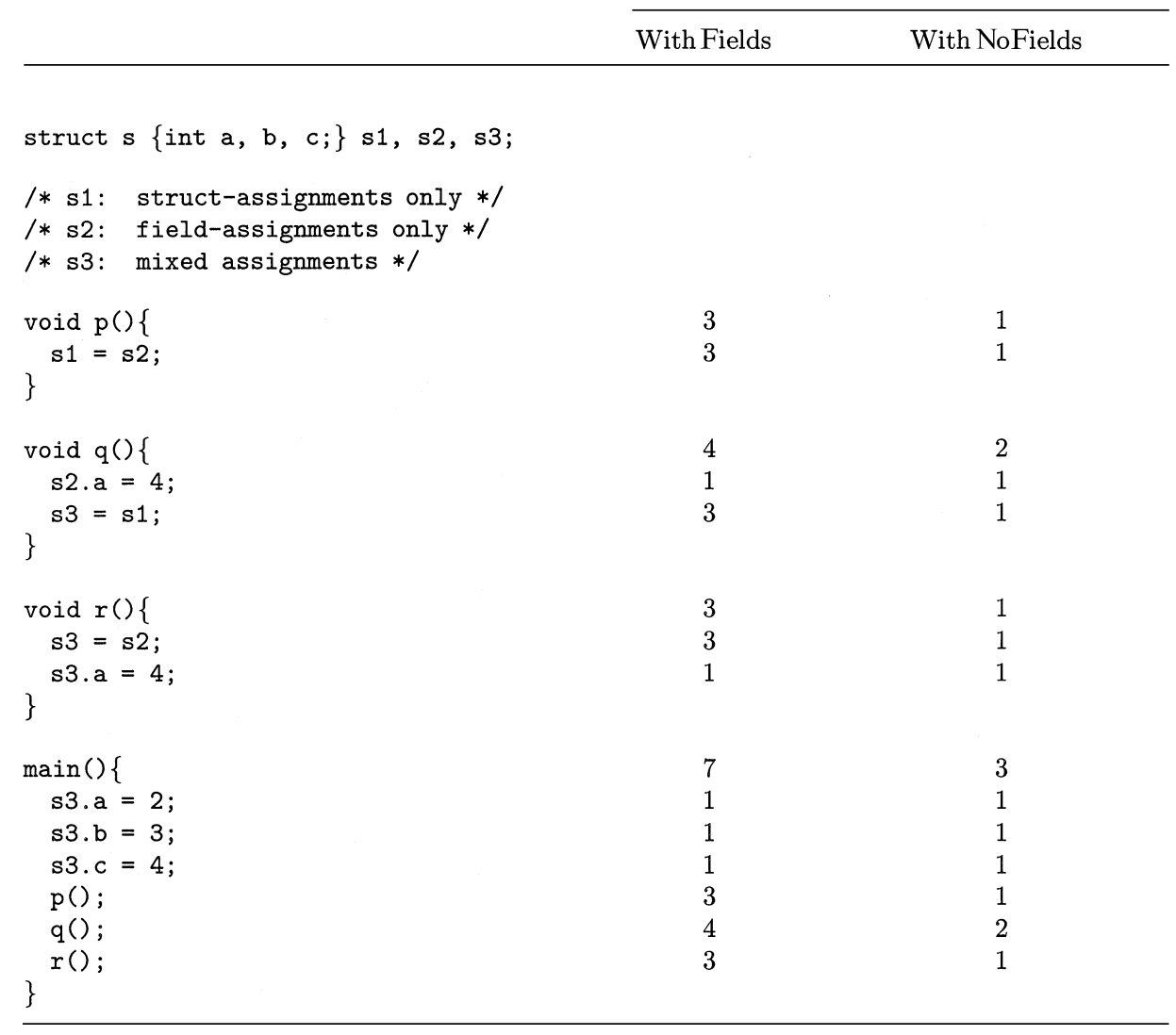

Fig. 14. Side effects counting example.

counting is used, when a field name experiences a side effect it is as if the entire struct experienced the effect, so that the call to $r$ reports 1 side effect (to struct s3), and the three assignments in main all are counted as causing a side effect to struct s3, rather than to its fields. Notice for the call of $\mathrm{q}$ in main only 2 fixed locations are reported as experiencing a side effect in NoFields counting, structs s3 and s2. The same counting is used for $M O D(P)$ sets.

Unions are handled in much the same manner as structs. If any member of a union appears in a $\mathrm{MOD}_{C}$ set, under Fields counting it will be expanded to all of its members. The actual internal representation of fields/members for structs and unions in FSAlias and FIAlias uses a start position and length; this sometimes allows recognition of exact overlap of two struct fields (or members) and results in better precision in counting.

There is also a problem with Fields counting of dynamically created structs if a user creates their own malloc. Recall that a unique heap creation site name is created for all cells corresponding to a single allocation statement in the code. In Figure 15, procedure a only stores into struct $* \mathrm{~s}$, and procedure b only 


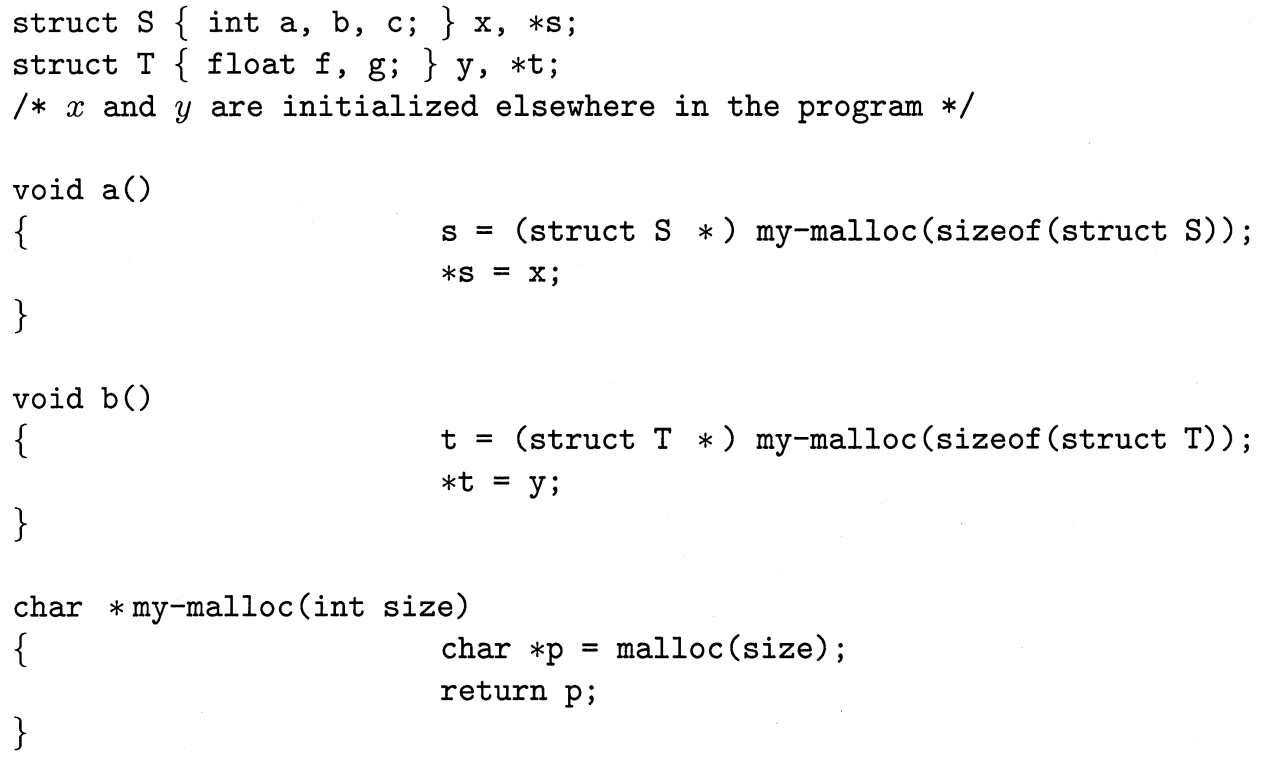

Fig. 15. Problem with Fields counting involving user-defined malloc.

stores into struct $* \mathrm{t}$; yet 5 fixed locations are reported in $M O D(\mathrm{a})$ and $M O D(\mathrm{~b})$, because the naming mechanism does not remember that the 5 fields come from two different structs. The problem is that the same heap creation site name for the malloc site in procedure my-malloc is used for all calling contexts. That name becomes associated with the sum of all the fields of all structs allocated using this procedure; effectively, encapsulation of the heap allocation within a procedure hides what is really going on from the aliasing algorithm.

NoFields counting is preferred, and the results in Section 4 are reported using this scheme, though Figure 24 offers results using Fields counting and shows how this choice can influence the results reported.

\section{EMPIRICAL RESULTS}

This section describes and discusses execution results of the $\mathrm{MOD}_{C}$ analyses. The $\mathrm{MOD}_{C}$, FSAlias, and FIAlias analysis code is implemented in C and analyzes a reduced version of $\mathrm{C}$ that excludes pointers to functions, setjump and longjump, but allows type casting and unions. Recall that because FSAlias as described does not handle function pointers, the implemented version of FIAlias does not handle function pointers either. None of the $\mathrm{C}$ programs we used as data contained function pointers. These results were gathered on a $75 \mathrm{MHz}$ processor Sun Sparcstation 20 with $348 \mathrm{MB}$ of RAM and 527MB swap space.

Table I shows information about the $45 \mathrm{C}$ programs that were analyzed. The programs are ordered by the number of ICFG nodes; this order is maintained in subsequent figures. The numbers of procedures, call statements, and assignment statements in each data program are shown. For $M O D(n)$, the relevant 
Table I. Program Data Set

\begin{tabular}{|c|c|c|c|c|c|c|c|}
\hline \multirow[b]{2}{*}{ Program } & \multirow[b]{2}{*}{ LOC } & \multirow[b]{2}{*}{ ICFG Nodes } & \multirow[b]{2}{*}{ \# Procs } & \multirow[b]{2}{*}{ \# Calls } & \multicolumn{2}{|c|}{ \# Assigns } & \multirow{2}{*}{$\begin{array}{l}\text { Flow-sens. } \\
\text { Alias Soln }\end{array}$} \\
\hline & & & & & All & Thru-deref & \\
\hline allroots & 215 & 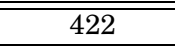 & $\overline{88}$ & 20 & $\overline{72}$ & 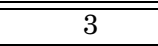 & $\overline{\sqrt{ }}$ \\
\hline fixoutput & 401 & 617 & 7 & 13 & 123 & 5 & $\sqrt{ }$ \\
\hline diffh & 1708 & 646 & 15 & 50 & 80 & 4 & $\sqrt{ }$ \\
\hline travel & 862 & 698 & 16 & 24 & 170 & 3 & $\sqrt{ }$ \\
\hline ul & 548 & 1027 & 15 & 36 & 168 & 6 & $\sqrt{ }$ \\
\hline plot2fig & 1495 & 1077 & 27 & 78 & 159 & 16 & $\sqrt{ }$ \\
\hline lex315 & 719 & 1297 & 18 & 103 & 137 & 6 & $\sqrt{ }$ \\
\hline compress & 1490 & 1319 & 16 & 29 & 274 & 11 & $\sqrt{ }$ \\
\hline clinpack & 1226 & 1429 & 14 & 80 & 267 & 30 & $\sqrt{ }$ \\
\hline loader & 1219 & 1563 & 31 & 80 & 242 & 78 & $\sqrt{ }$ \\
\hline mway & 705 & 1576 & 22 & 43 & 406 & 71 & $\sqrt{ }$ \\
\hline ansitape & 1596 & 1747 & 36 & 110 & 274 & 21 & $\sqrt{ }$ \\
\hline stanford & 887 & 1771 & 48 & 80 & 369 & 42 & $\sqrt{ }$ \\
\hline pokerd & 1243 & 1895 & 27 & 86 & 296 & 59 & $\sqrt{ }$ \\
\hline zipship & 1283 & 1955 & 14 & 53 & 332 & 59 & $\sqrt{ }$ \\
\hline dixie & 2109 & 2341 & 36 & 83 & 394 & 73 & $\sqrt{ }$ \\
\hline zipnote & 3155 & 2407 & 20 & 71 & 348 & 86 & $\sqrt{ }$ \\
\hline learn & 1483 & 2626 & 36 & 80 & 432 & 59 & $\sqrt{ }$ \\
\hline xmodem & 1712 & 2672 & 28 & 156 & 447 & 97 & $\sqrt{ }$ \\
\hline compiler & 2232 & 3008 & 39 & 350 & 304 & 2 & $\sqrt{ }$ \\
\hline zipcloak & 3644 & 3033 & 30 & 93 & 424 & 104 & $\sqrt{ }$ \\
\hline $\operatorname{sim}$ & 1439 & 3034 & 17 & 29 & 818 & 130 & $\sqrt{ }$ \\
\hline cdecl & 1015 & 3196 & 33 & 204 & 448 & 25 & $\sqrt{ }$ \\
\hline diff & 1708 & 3300 & 43 & 129 & 569 & 100 & $\sqrt{ }$ \\
\hline unzip & 4106 & 3416 & 40 & 99 & 731 & 52 & $\sqrt{ }$ \\
\hline assembler & 2673 & 3601 & 53 & 248 & 533 & 233 & $\sqrt{ }$ \\
\hline gnugo & 2901 & 3651 & 29 & 89 & 650 & 109 & $\sqrt{ }$ \\
\hline live & 1886 & 4101 & 87 & 204 & 885 & 243 & $\sqrt{ }$ \\
\hline lharc & 3303 & 4250 & 87 & 198 & 791 & 123 & $\sqrt{ }$ \\
\hline patch & 2672 & 4608 & 56 & 271 & 750 & 135 & $\sqrt{ }$ \\
\hline simulator & 3733 & 5574 & 100 & 409 & 666 & 107 & $\sqrt{ }$ \\
\hline $\operatorname{arc}$ & 7507 & 5856 & 96 & 237 & 1105 & 160 & $\sqrt{ }$ \\
\hline triangle & 1930 & 6119 & 19 & 43 & 1072 & 241 & $\sqrt{ }$ \\
\hline tbl & 2511 & 6162 & 85 & 316 & 907 & 279 & $\sqrt{ }$ \\
\hline football & 2222 & 7313 & 59 & 258 & 847 & 225 & $\sqrt{ }$ \\
\hline flex & 6970 & 7376 & 86 & 307 & 1505 & 248 & $\sqrt{ }$ \\
\hline zip & 7427 & 9288 & 109 & 324 & 1554 & 331 & \\
\hline $072 . s c$ & 8087 & 13690 & 154 & 698 & 1826 & 201 & \\
\hline spim & 19032 & 16740 & 168 & 974 & 1566 & 374 & \\
\hline larn & 9546 & 21184 & 264 & 2218 & 2536 & 158 & \\
\hline tsl & 14646 & 27302 & 450 & 2109 & 2350 & 587 & \\
\hline 008.espresso & 13567 & 30510 & 308 & 1830 & 5054 & 1524 & \\
\hline moria & 24596 & 38572 & 432 & 3708 & 5893 & 1493 & $\sqrt{ }$ \\
\hline TWMC & 23833 & 51627 & 204 & 796 & 10669 & 3949 & \\
\hline nethack & 28735 & 58317 & 474 & 2837 & 4268 & 900 & \\
\hline
\end{tabular}


statements in a program are assignments and call statements. Assignments through a pointer dereference are distinguished because these assignments have nontrivial solutions, whereas other assignments (e.g., $i=0 ;$ ) have trivial solutions. For $\operatorname{MOD}_{C}(P)$ the procedures are the relevant program constructs.

The last column of Table I indicates whether or not FSAlias succeeds in calculating an alias solution for the program. FSAlias is unable to calculate a solution for 8 of the programs because it runs out of virtual memory; FIAlias can calculate a solution for all the programs. This raises the question of what characteristics of a program affect the availability of a flow-/context-sensitive alias solution. Certainly program size is a factor because of the relationship between size of solution and size of program, but it cannot be the only factor as the contrasting results for moria and zip show. Moria is a "large" program with an FSAlias solution. Nor do the size differences between zip and flex seem vast enough to indicate a threshold on the power of FSAlias. The root of the problem is caused by recursive data structures. The FSAlias algorithm uses the somewhat naive $k$-limiting approximation to handle recursive data structures; however, in the cases of these larger programs that make excessive use of recursive data structures, the analysis gets bogged down in the generation and propagation of k-limited aliases, using much memory. Moria has no recursive data structures. Zip uses recursive data structures much more heavily than flex.

$\mathrm{MOD}_{C}$ precision is reported in terms of the average number of fixed locations reported modified per kind of statement. The $\mathrm{MOD}_{C}($ FSAlias $)$ solution is always a subset of the $\mathrm{MOD}_{C}$ (FIAlias) solution, and both are safe, so that extra modifications reported by the $\operatorname{MOD}_{C}$ (FIAlias) solution are spurious. The raw data used to produce the figures in the following sections can be found in Appendix C.

\subsection{Precision at Procedures and Call Statements}

Figures 16(a) and 16(b) report the average numbers of fixed locations modified by procedures for both $\operatorname{MOD}_{C}$ (FSAlias) and $\operatorname{MOD}_{C}$ (FIAlias). The $\mathrm{MOD}_{C}$ (FIAlias) result for moria is elided because it skews the figure; the raw numbers are presented instead. The bars for each program are divided into the kind of location being modified. Each segment of the average bar is the average over the numbers in Figure 16 for that kind of location. Formal parameters are reported as locals. Notice the enhanced precision of flow and context sensitivity (especially for the large program moria); $\operatorname{MOD}_{C}($ FSAlias) reports 11 fixed locations modified on average by procedures, against the 17 fixed locations reported by $\mathrm{MOD}_{C}$ (FIAlias) ${ }^{14}$ Also notice that the average number of fixed locations modified is not closely correlated to program size.

Figure 16(c) shows the same results for the programs with only a flow-/ context-insensitive $\mathrm{MOD}_{C}$ solution. Figure 16(d) compares the average totals from Figures 16(a) and 16(b) in a more visually apparent manner. Programs are plotted by their sizes in ICFG nodes, along the x-axis. Again, moria is excluded from this comparison because it skews the figure.

${ }^{14}$ Excluding moria's result, $\mathrm{MOD}_{C}$ (FIAlias) reports 14 fixed locations modified on average. 


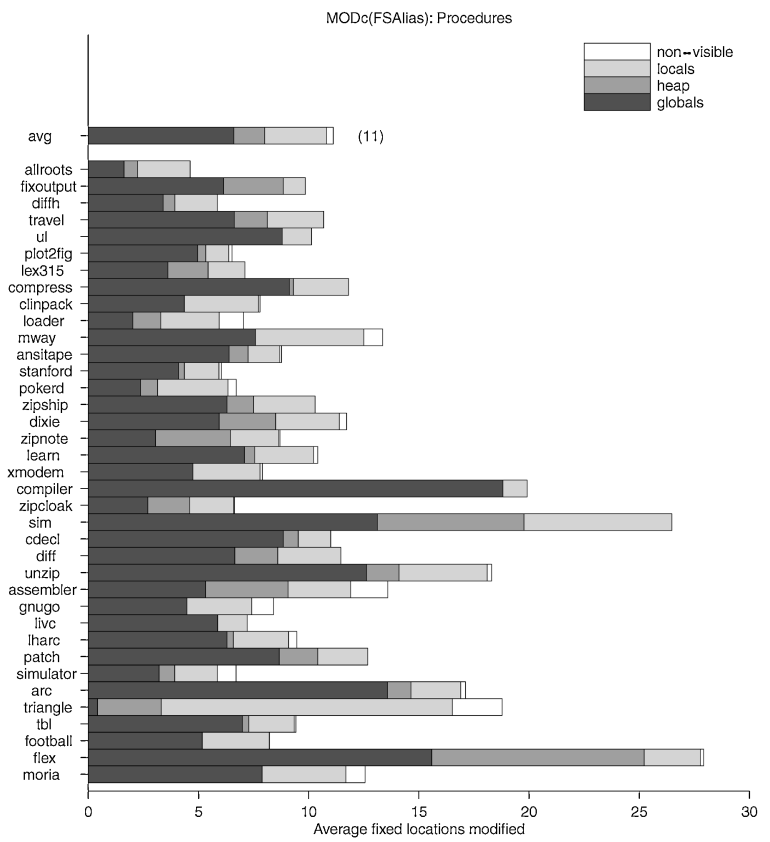

(a)

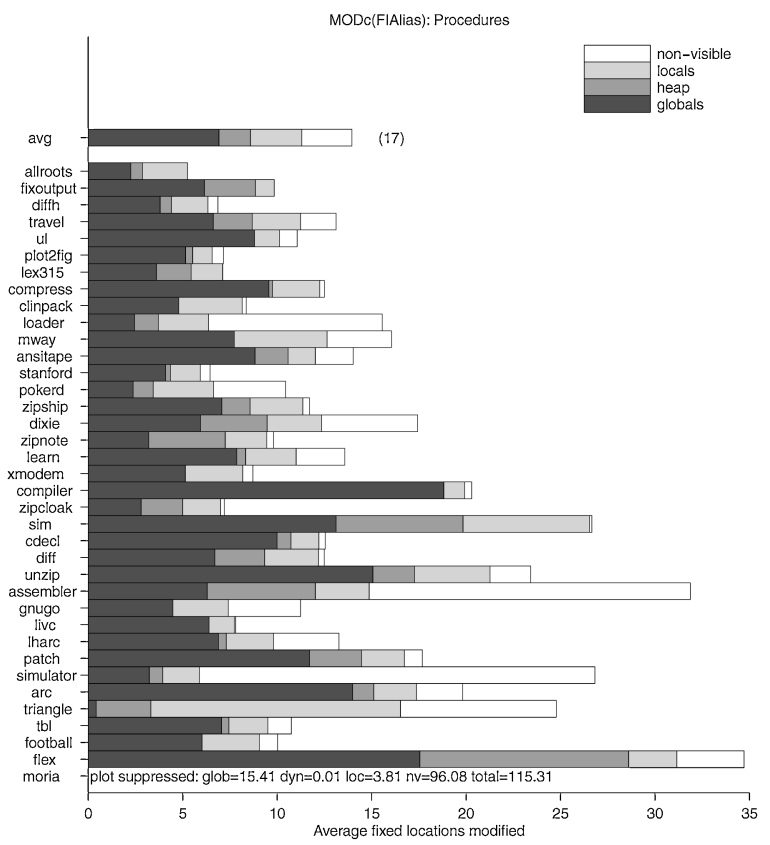

(b)

Fig. 16. Average fixed locations modified by procedures. 


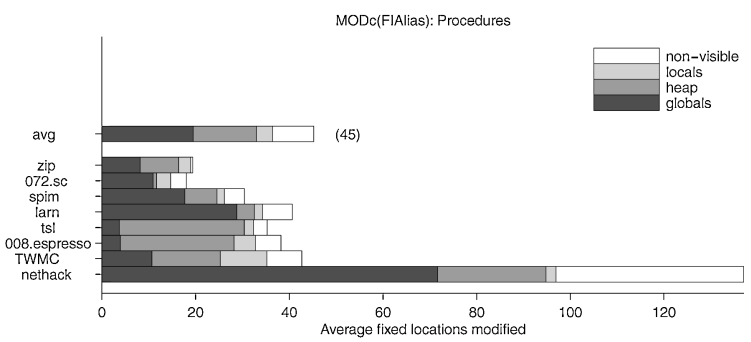

(c)

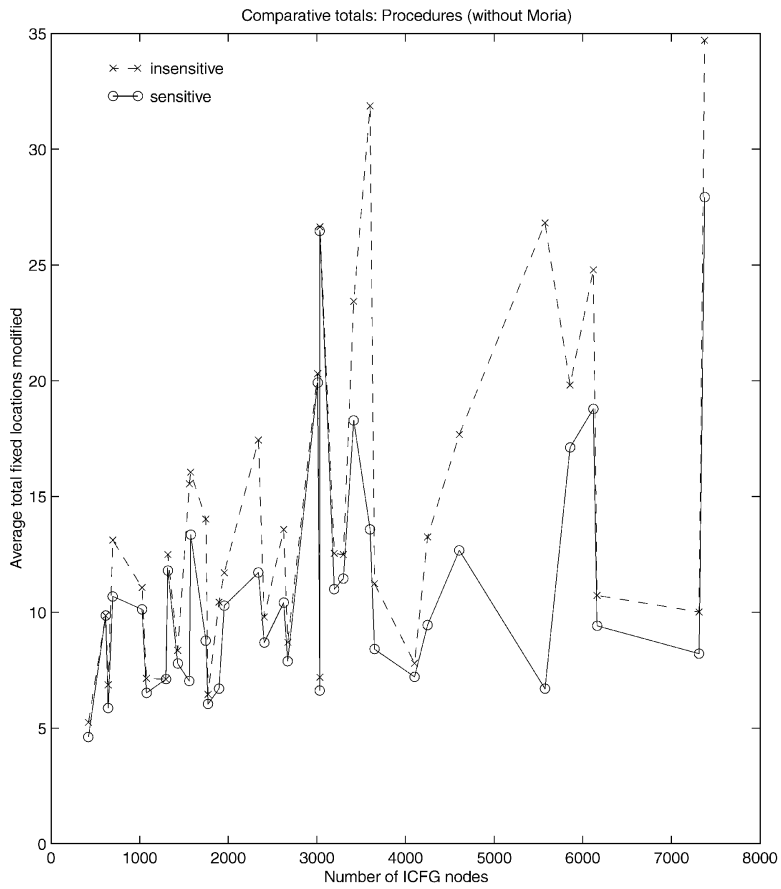

(d)

Fig. 16. Continued.

Figures 17(a) and 17(b) present the same information as Figures 16(a) and 16(b) for fixed locations modified by call statements. The conclusions to be drawn are similar. The procedure averages in Figures 16(a) and 16(b) contain the effects of all calls to the procedure. Recall that $\mathrm{MOD}_{C}$ for procedure $P$ is defined to be the union of $P M O D(P, R A)$ over all $R A$ 's that occur. At a call site, however, only the effects at that call site are reported. So we expect $\mathrm{MOD}_{C}$ for procedure $P$ on average to be higher than $\mathrm{MOD}_{C}$ at a call site.

Figure 18 shows the standard deviations for the $\operatorname{MOD}_{C}($ FSAlias $)$ results previously reported in Figure 16 (i.e., the number of fixed locations possibly modified). These standard deviations are typical of those for each of our observations in these experiments; they are included for completeness. 


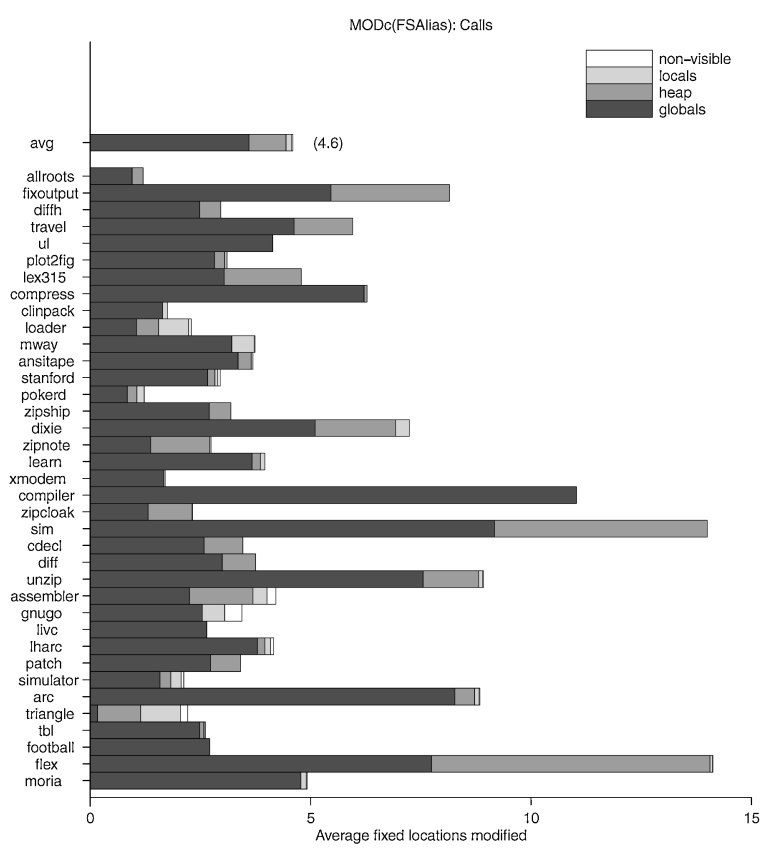

(a)

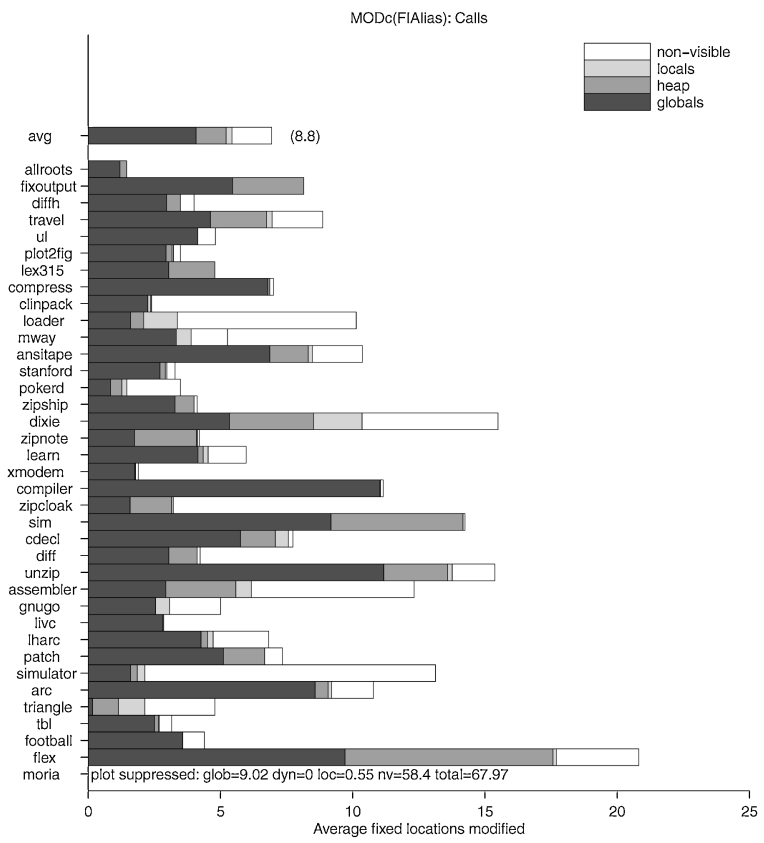

(b)

Fig. 17. Average fixed locations modified by call statements. ACM Transactions on Programming Languages and Systems, Vol. 23, No. 1, March 2001. 


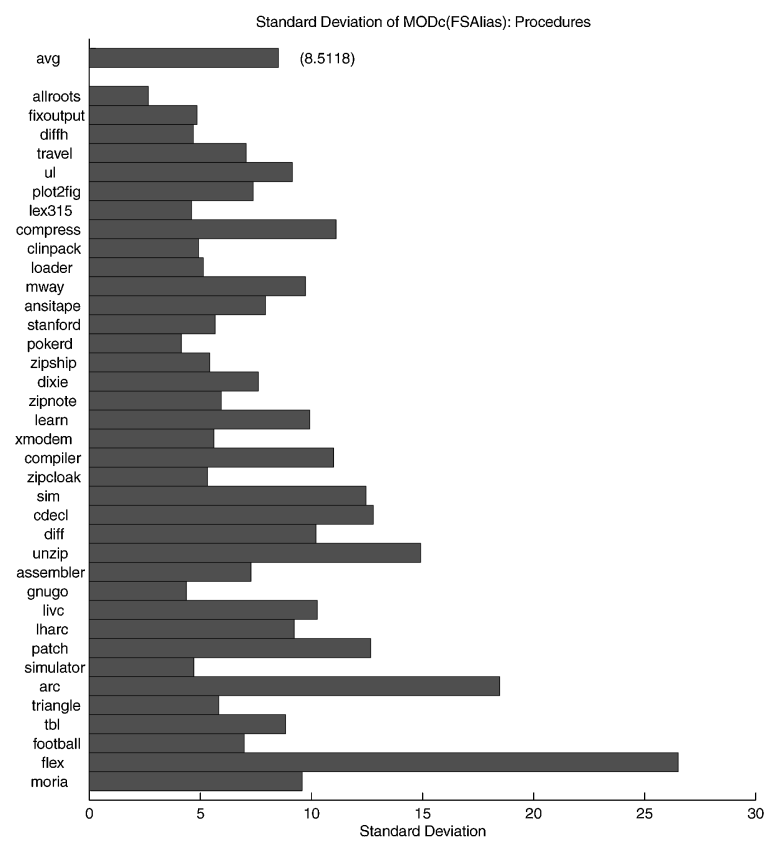

Fig. 18. Procedure $\operatorname{MOD}_{C}($ FSAlias $)$ standard deviations.

Figure 19 addresses the comparative difference between the $\operatorname{MOD}_{C}($ FSAlias $)$ and $\operatorname{MOD}_{C}($ FIAlias $)$ solutions. If sens is the number of fixed locations reported modified at a program point by $\operatorname{MOD}_{C}($ FSAlias $)$, and insens is the number reported by $\mathrm{MOD}_{C}$ (FIAlias) at that same point, then the relative mean is the average of the calculation (insens - sens)/insens over relevant program points in a program. Figure 19 shows the relative means for procedure side effects (Figure 19(a)) and call statement side effects (Figure 19(b)). These measurements indicate the proportion of the $\mathrm{MOD}_{C}$ (FIAlias) solution that must be in error. Low numbers here mean that the $\operatorname{MOD}_{C}($ FIAlias $)$ solution is nearly as precise as the $\mathrm{MOD}_{C}($ FSAlias) solution. Zero means that the solutions are the same. The average bars are calculated by treating all the program points in all programs as one set and averaging over all of them, rather than taking the average of the averages for each program. Also note that summing the relative means at each program point and averaging is substantially different from using the relative means calculation on the average total fixed locations modified results from Figures 16 and 17. For example, consider a procedure with two statements, where for the first statement $\operatorname{MOD}_{C}($ FSAlias $)$ reports 1 location modified and $\operatorname{MOD}_{C}($ FIAlias $)$ reports 2 locations modified, and for the second statement $\operatorname{MOD}_{C}($ FSAlias $)$ reports 99 locations modified and $\mathrm{MOD}_{C}($ FIAlias $)$ reports 100 locations modified. The average total locations modified by the procedure as reported by $\operatorname{MOD}_{C}($ FSAlias $)$ is 50 , and by $\operatorname{MOD}_{C}($ FIAlias $)$ is 51 . The relative mean for the first statement is 0.5 , and the relative mean for the second is 0.01 . The relative mean for the whole procedure is then 0.255 , but the relative mean calculation using the average total numbers is $1 / 51$ (a little less 


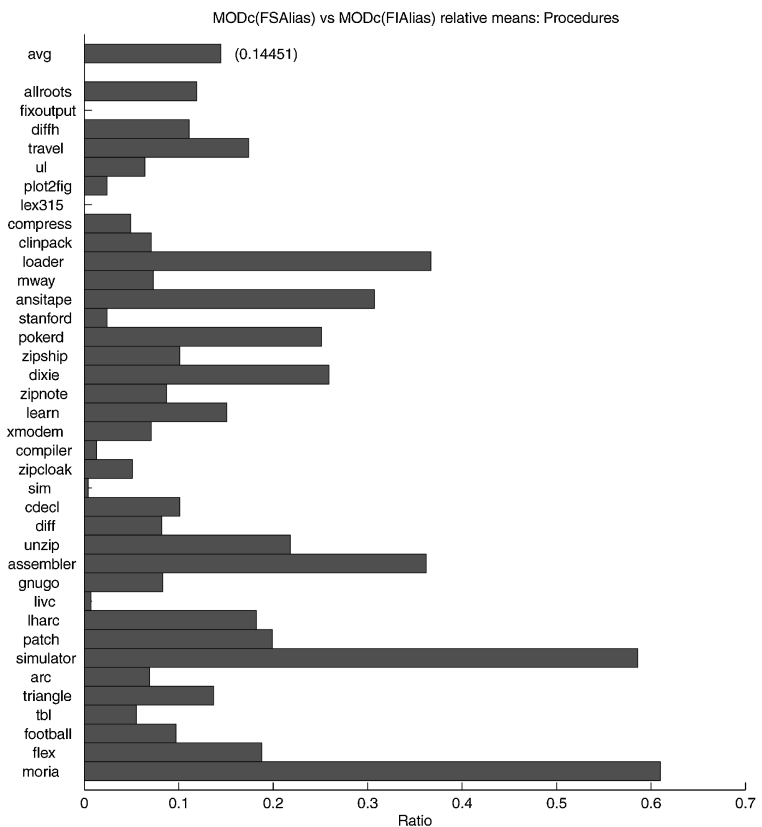

(a)

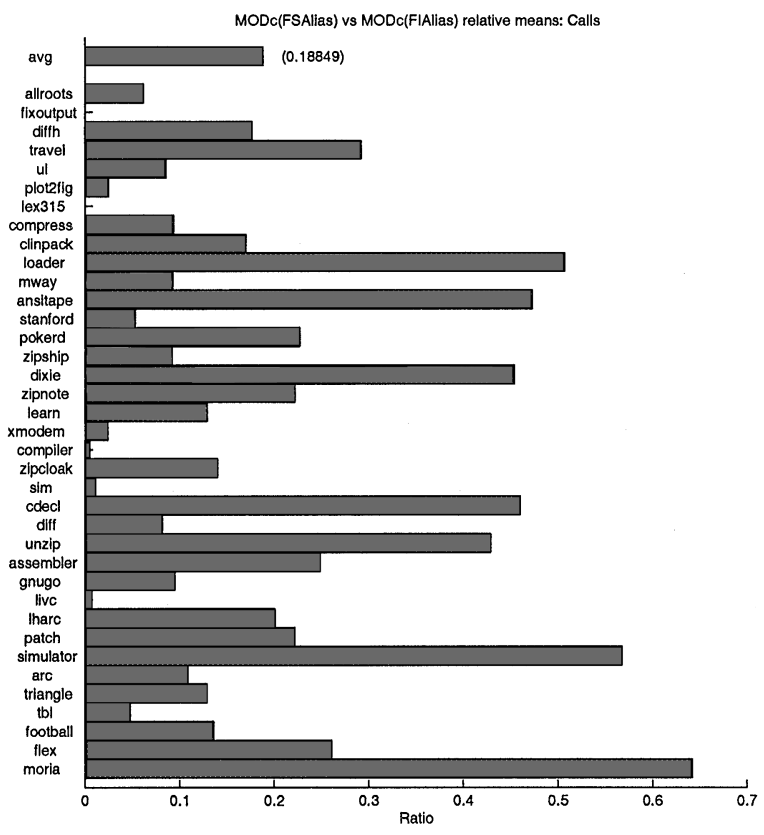

(b)

Fig. 19. Relative means for procedures and call statements. 
than 0.02). The numbers in Figure 19 are calculated using the former approach. These results give an indication of the trade-off in precision involved in using the flow-/context-insensitive analysis.

Even so, the precision of the $\mathrm{MOD}_{C}$ (FIAlias) analysis is not to be understated. Figure 20 shows the average proportion of reported fixed locations modified by procedures and calls to the number of fixed locations potentially modified. The number of fixed locations potentially modified at a call or assignment statement is the sum of the number of globals in the program, the number of dynamic allocation sites in the program, the number of locals in the enclosing procedure, and the number of accessible non-visibles. The number of fixed locations potentially modified by a procedure is the sum of the numbers of potentially modified locations for each assignment and call statement in the procedure. Figure 20 shows what percentage the average totals reported by $\operatorname{MOD}_{C}($ FIAlias $)$ are of this worst case. In parentheses after each bar is the average total number of fixed locations reported modified by $\mathrm{MOD}_{C}($ FIAlias $)$. Very low percentages indicate that the worst-case $\mathrm{MOD}_{C}$ solution is very much larger than what can be calculated using even flow-/context-insensitive data-flow analysis and indicate the significant advantages that inexpensive data-flow analysis can offer.

Table II presents another view comparing $\operatorname{MOD}_{C}($ FSAlias $)$ and $\mathrm{MOD}_{C}($ FIAlias $)$ that shows a frequency table of the numbers of procedures that modify a certain number of fixed locations. All the procedures in all the programs are considered together when constructing these frequency tables. ${ }^{15}$ The frequency tables are broken down by fixed-location kind with the Total column representing all kinds. For example, Table II (a) says that 367 of the procedures in all the programs modify 0 globals (Glob) and 132 procedures modify no fixed locations (Total). The Percent Below column shows what percentage of the procedures have that many total side effects or more. Gaps in the sequence indicate that there are no procedures with that many side effects. The reports of procedures modifying 0 total fixed locations are not erroneous, but arise from simple procedures which have no local variables and no side effects (e.g., absolute value function).

The frequency tables for each kind of fixed location and for the totals seem to approximate a half normal distribution. For $\mathrm{MOD}_{C}($ FSAlias $)$, the frequency table for globals is the most spread out. This is probably an artifact of how globals are used in the data programs. For $\operatorname{MOD}_{C}($ FIAlias $)$, the frequency table for non-visibles is the most spread out. The $\operatorname{MOD}_{C}$ (FIAlias) frequency tables for all types of fixed locations apart from non-visibles appear to be close to the shape of the frequency tables for $\mathrm{MOD}_{C}$ (FSAlias), albeit flatter and longer, but the $\operatorname{MOD}_{C}$ (FIAlias) frequency table for non-visibles is much worse. Since $\operatorname{MOD}_{C}($ FSAlias $)$ is safe, this cannot be an artifact of the real solution. It appears that when the $\operatorname{MOD}_{C}($ FIAlias $)$ solution gets overly approximate, it is with regard to non-visibles. Why this is so and what methods should be adopted to remedy the situation are open questions.

\footnotetext{
${ }^{15}$ The programs with no FSAlias solution are omitted when calculating the $\mathrm{MOD}_{C}($ FIAlias $)$ frequency table.
} 


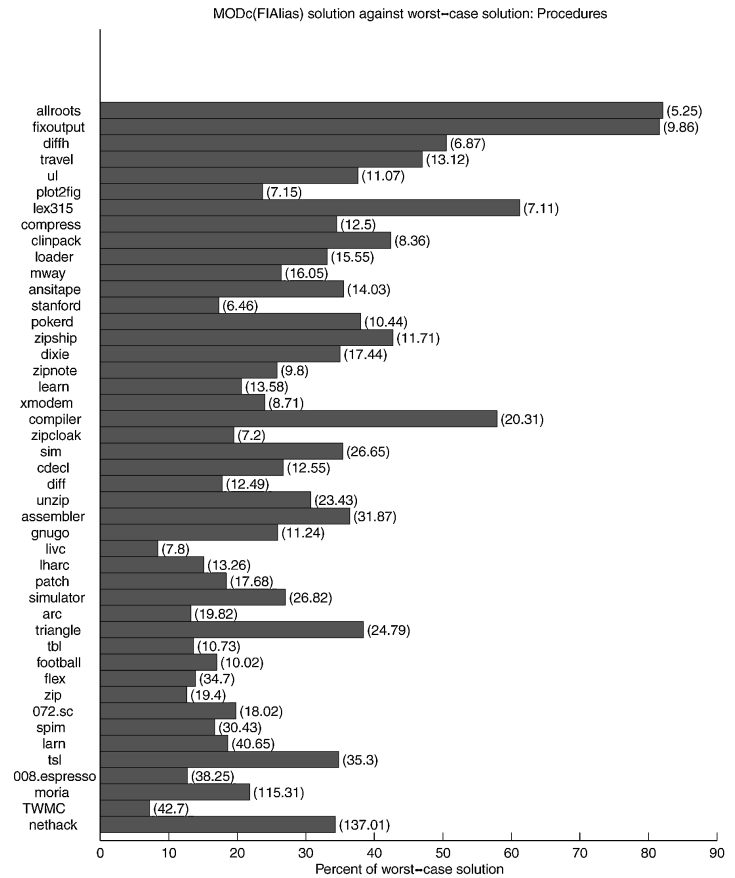

(a)

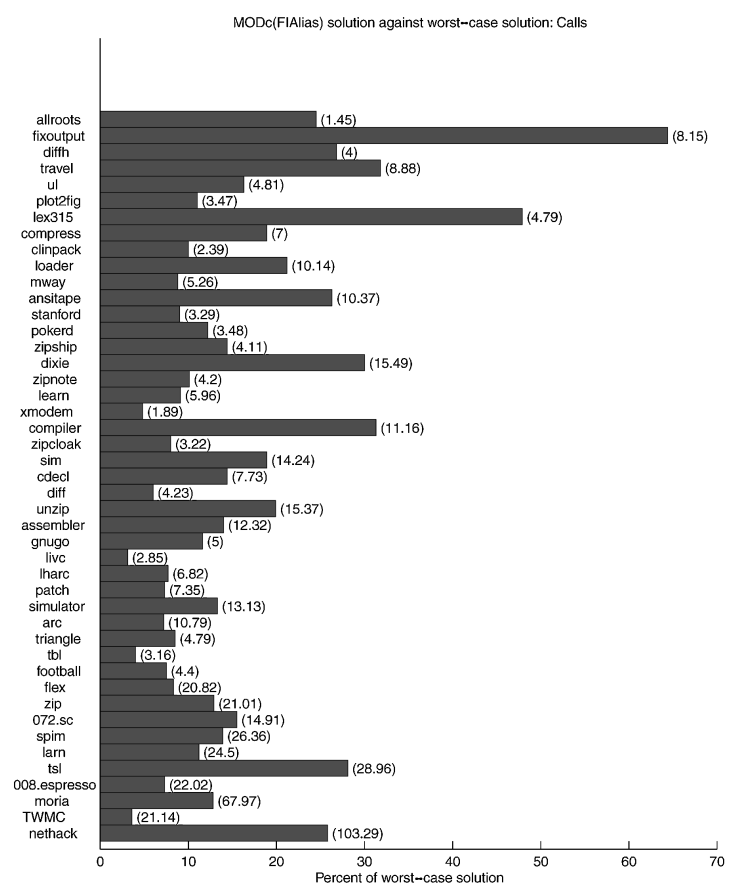

(b)

Fig. 20. $\operatorname{MOD}_{C}($ FIAlias $)$ solution as percentage of worst-case solution. 
Table II. Procedure Frequency Tables

\begin{tabular}{|r|rrrr|r|r|}
\hline \# Side & & & & & & Percent \\
Effects & Glob & Dyn & Loc & Nv & Total & Below \\
\hline \hline 0 & 367 & 1389 & 577 & 1624 & 132 & 100.00 \\
1 & 422 & 117 & 348 & 87 & 224 & 92.77 \\
2 & 175 & 119 & 239 & 32 & 181 & 80.49 \\
3 & 181 & 66 & 160 & 25 & 133 & 70.58 \\
4 & 133 & 31 & 132 & 19 & 139 & 63.29 \\
5 & 51 & 9 & 79 & 4 & 104 & 55.67 \\
6 & 64 & 5 & 60 & 9 & 120 & 49.97 \\
7 & 34 & 12 & 48 & 1 & 65 & 43.40 \\
8 & 50 & 9 & 37 & 3 & 58 & 39.84 \\
9 & 21 & 2 & 29 & 3 & 69 & 36.66 \\
$10-12$ & 53 & 28 & 57 & 6 & 123 & 32.88 \\
$13-15$ & 28 & 7 & 27 & 2 & 98 & 26.14 \\
$16-18$ & 38 & 18 & 14 & 2 & 54 & 20.77 \\
$19-21$ & 35 & 3 & 8 & 1 & 43 & 17.81 \\
$22-24$ & 22 & 2 & 5 & 2 & 35 & 15.45 \\
$25-27$ & 14 & 1 & 0 & 3 & 27 & 13.53 \\
$28-30$ & 14 & 1 & 1 & 0 & 34 & 12.05 \\
$31-33$ & 10 & 0 & 0 & 0 & 27 & 10.19 \\
$34-36$ & 12 & 0 & 2 & 0 & 12 & 8.71 \\
$37-39$ & 4 & 2 & 0 & 1 & 18 & 8.05 \\
$40-42$ & 3 & 0 & 2 & 0 & 12 & 7.07 \\
$43-45$ & 12 & 0 & 0 & 0 & 12 & 6.41 \\
$46-48$ & 38 & 0 & 0 & 0 & 17 & 5.75 \\
$49-51$ & 1 & 0 & 0 & 0 & 11 & 4.82 \\
$52-54$ & 6 & 0 & 0 & 0 & 10 & 4.22 \\
$55-57$ & 1 & 1 & 0 & 0 & 8 & 3.67 \\
$58-60$ & 5 & 0 & 0 & 0 & 7 & 3.23 \\
$61-63$ & 2 & 0 & 0 & 0 & 5 & 2.85 \\
$64-66$ & 5 & 0 & 0 & 1 & 9 & 2.58 \\
$67-69$ & 3 & 0 & 0 & 0 & 9 & 2.08 \\
$70-72$ & 0 & 0 & 0 & 0 & 3 & 1.59 \\
$73-75$ & 2 & 3 & 0 & 0 & 1 & 1.42 \\
$76-78$ & 6 & 0 & 0 & 0 & 3 & 1.37 \\
$79-81$ & 2 & 0 & 0 & 0 & 3 & 1.21 \\
$82-84$ & 2 & 0 & 0 & 0 & 0 & 1.04 \\
$85-87$ & 3 & 0 & 0 & 0 & 6 & 1.04 \\
$88-90$ & 0 & 0 & 0 & 0 & 2 & 0.71 \\
$94-96$ & 0 & 0 & 0 & 0 & 1 & 0.60 \\
$106-108$ & 2 & 0 & 0 & 0 & 0 & 0.55 \\
$109-111$ & 1 & 0 & 0 & 0 & 0 & 0.55 \\
$115-117$ & 0 & 0 & 0 & 0 & 1 & 0.55 \\
$118-120$ & 0 & 0 & 0 & 0 & 1 & 0.49 \\
$121-123$ & 0 & 0 & 0 & 0 & 1 & 0.44 \\
$124-126$ & 0 & 0 & 0 & 0 & 2 & 0.38 \\
$127-129$ & 0 & 0 & 0 & 0 & 1 & 0.27 \\
$151-157$ & 0 & 0 & 0 & 0 & 1 & 0.22 \\
\hline 228 & 3 & 0 & 0 & 0 & 0 & 0.16 \\
& 0 & 0 & 0 & 0 & 3 & 0.16 \\
\hline
\end{tabular}

$\operatorname{MOD}_{C}($ FSAlias $)$

(a)

ACM Transactions on Programming Languages and Systems, Vol. 23, No. 1, March 2001. 
Table II. Continued

\begin{tabular}{|r|rrrr|r|r|}
\hline \# Side & & & & & & Percent \\
Effects & Glob & Dyn & Loc & Nv & Total & Below \\
\hline \hline 0 & 351 & 1344 & 577 & 935 & 113 & 100.00 \\
1 & 375 & 92 & 348 & 117 & 216 & 93.81 \\
2 & 143 & 96 & 239 & 76 & 172 & 81.97 \\
3 & 172 & 101 & 160 & 71 & 87 & 72.55 \\
4 & 121 & 59 & 132 & 33 & 84 & 67.78 \\
5 & 48 & 12 & 78 & 29 & 69 & 63.18 \\
6 & 59 & 9 & 60 & 44 & 64 & 59.40 \\
7 & 30 & 17 & 49 & 33 & 34 & 55.89 \\
8 & 35 & 9 & 37 & 45 & 36 & 54.03 \\
9 & 32 & 5 & 29 & 19 & 44 & 52.05 \\
$10-19$ & 132 & 66 & 100 & 83 & 278 & 49.64 \\
$20-29$ & 151 & 8 & 12 & 117 & 164 & 34.41 \\
$30-39$ & 74 & 1 & 2 & 20 & 110 & 25.42 \\
$40-49$ & 19 & 2 & 2 & 21 & 63 & 19.40 \\
$50-59$ & 12 & 1 & 0 & 1 & 44 & 15.95 \\
$60-69$ & 44 & 0 & 0 & 6 & 21 & 13.53 \\
$70-79$ & 10 & 3 & 0 & 7 & 19 & 12.38 \\
$80-89$ & 8 & 0 & 0 & 5 & 18 & 11.34 \\
$90-99$ & 0 & 0 & 0 & 0 & 10 & 10.36 \\
$100-109$ & 5 & 0 & 0 & 3 & 4 & 9.81 \\
$110-119$ & 1 & 0 & 0 & 2 & 5 & 9.59 \\
$120-129$ & 0 & 0 & 0 & 2 & 3 & 9.32 \\
$130-139$ & 0 & 0 & 0 & 0 & 3 & 9.15 \\
$140-149$ & 0 & 0 & 0 & 28 & 4 & 8.99 \\
$150-159$ & 3 & 0 & 0 & 5 & 1 & 8.77 \\
$160-169$ & 0 & 0 & 0 & 1 & 0 & 8.71 \\
$170-179$ & 0 & 0 & 0 & 36 & 21 & 8.71 \\
$180-189$ & 0 & 0 & 0 & 7 & 10 & 7.56 \\
$190-199$ & 0 & 0 & 0 & 3 & 1 & 7.01 \\
$200-209$ & 0 & 0 & 0 & 7 & 21 & 6.96 \\
$210-219$ & 0 & 0 & 0 & 3 & 18 & 5.81 \\
$220-229$ & 0 & 0 & 0 & 3 & 6 & 4.82 \\
$230-239$ & 0 & 0 & 0 & 2 & 7 & 4.49 \\
$240-249$ & 0 & 0 & 0 & 3 & 6 & 4.11 \\
$250-259$ & 0 & 0 & 0 & 4 & 6 & 3.78 \\
$260-269$ & 0 & 0 & 0 & 2 & 0 & 3.45 \\
$270-279$ & 0 & 0 & 0 & 1 & 2 & 3.45 \\
$280-289$ & 0 & 0 & 0 & 1 & 4 & 3.34 \\
$290-299$ & 0 & 0 & 0 & 0 & 2 & 3.12 \\
$300-309$ & 0 & 0 & 0 & 0 & 2 & 3.01 \\
$310-319$ & 0 & 0 & 0 & 6 & 2 & 2.90 \\
$320-329$ & 0 & 0 & 0 & 2 & 1 & 2.79 \\
$340-349$ & 0 & 0 & 0 & 1 & 0 & 2.74 \\
$350-359$ & 0 & 0 & 0 & 18 & 3 & 2.74 \\
$360-369$ & 0 & 0 & 0 & 6 & 3 & 2.58 \\
$370-379$ & 0 & 0 & 0 & 4 & 2 & 2.41 \\
$380-389$ & 0 & 0 & 0 & 5 & 0 & 2.30 \\
$390-399$ & 0 & 0 & 0 & 2 & 0 & 2.30 \\
$400-409$ & 0 & 0 & 0 & 3 & 0 & 2.30 \\
$410-419$ & 0 & 0 & 0 & 3 & 16 & 2.30 \\
\hline
\end{tabular}

$\operatorname{MOD}_{C}($ FIAlias $)$

ACM Transactions on Programming Languages and Systems, Vol. 23, No. 1, March 2001. 
Table II. Continued

\begin{tabular}{|l|cccc|c|c|}
\hline $\begin{array}{l}\text { \# Side } \\
\text { Effects }\end{array}$ & Glob & Dyn & Loc & Nv & Total & $\begin{array}{c}\text { Percent } \\
\text { Below }\end{array}$ \\
\hline \hline $420-429$ & 0 & 0 & 0 & 0 & 1 & 1.42 \\
$430-439$ & 0 & 0 & 0 & 0 & 3 & 1.37 \\
$440-449$ & 0 & 0 & 0 & 0 & 6 & 1.21 \\
$450-459$ & 0 & 0 & 0 & 0 & 8 & 0.88 \\
$470-479$ & 0 & 0 & 0 & 0 & 2 & 0.44 \\
$480-489$ & 0 & 0 & 0 & 0 & 2 & 0.33 \\
$490-499$ & 0 & 0 & 0 & 0 & 1 & 0.22 \\
$510-519$ & 0 & 0 & 0 & 0 & 3 & 0.16 \\
\hline
\end{tabular}

\subsection{Precision at Through-Dereference Statements}

Figures 21(a) and 21(b) show the average numbers of fixed locations modified by through-dereference assignment statements, similarly to Figure 16 . These results are not discussed at length here because they are more related to the choice of aliasing algorithm than the $\mathrm{MOD}_{C}$ algorithm. Nevertheless, their precision is interesting.

Any executable assignment in a normally terminating program will modify at least one fixed location. Thus, 1 is a lower bound of total fixed locations modified per assignment statement (the dotted lines in Figures 21(a) and 21(b) show the line $x=1)$. The precision of these results for $\mathrm{MOD}_{C}$ (FSAlias) is very encouraging, and highlights the precision of the flow-/context-sensitive algorithm. The totals are all close to 1 with a maximum value of 2 and an average of 1.3 . In contrast, $\operatorname{MOD}_{C}$ (FIAlias) is more imprecise, averaging 5.1, and sometimes being wildly inaccurate as in such cases as moria. ${ }^{16}$ Moria is a large program with very many large (though nonrecursive) data structures with several aliases. Perhaps the inability of the $\mathrm{MOD}_{C}($ FIAlias $)$ algorithm to distinguish calling context and its inability to kill aliases explain the massive distortion between the $\operatorname{MOD}_{C}$ (FSAlias) and $\operatorname{MOD}_{C}$ (FIAlias) solutions for moria.

\subsection{Discussion of Precision}

A safe $\mathrm{MOD}_{C}$ solution is traditionally defined to be a pointwise superset of the precise solution. In other words, any fixed location that can be modified by some execution is guaranteed to be in the computed solution. The equations in Section 3.1 produce a safe solution. A reverse-safe solution is one that is pointwise a subset of the precise solution. Barth [1978] defines the concept precise up to symbolic execution to mean precise assuming that all program branches are executable; effectively, this means that all intraprocedural paths are considered to be executable. It is possible to define reverse-safe up to symbolic execution. This is done by ensuring the approximate solution is a pointwise subset of the precise-up-to-symbolic-execution solution.

There are two major sources of imprecision. The first is due to intraprocedural paths that cannot be executed. The second is due to various interactions of

${ }^{16}$ Without moria the average total for $\operatorname{MOD}_{C}($ FIAlias $)$ is 2.6. 


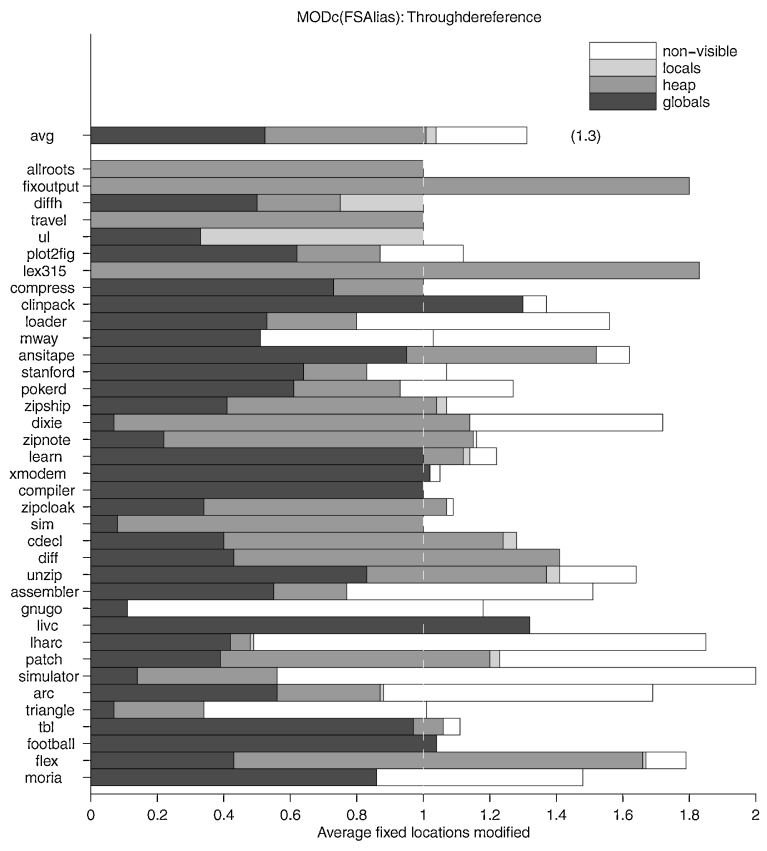

(a)

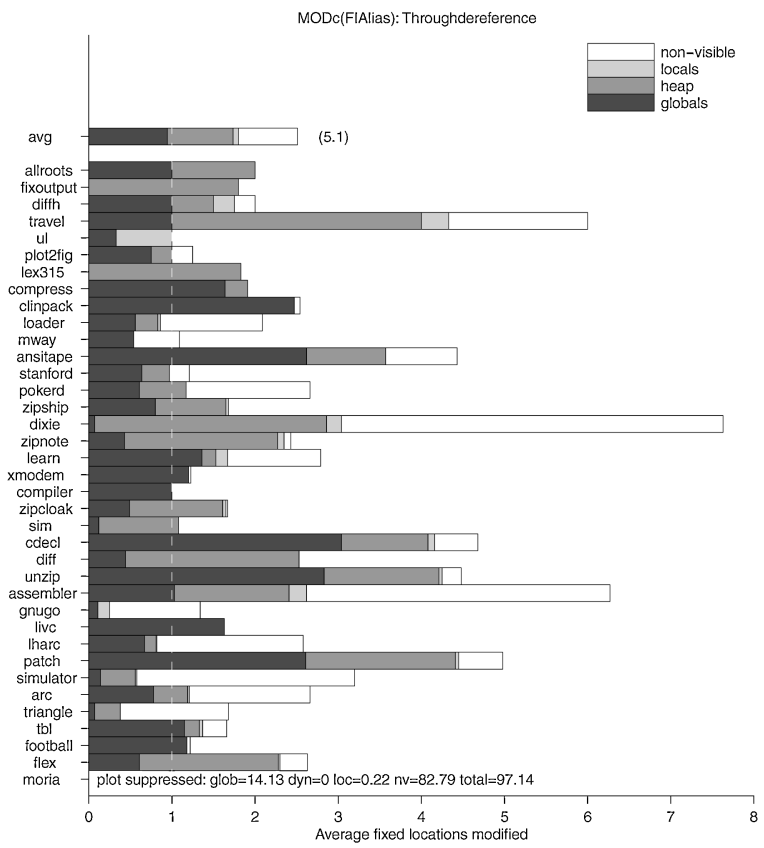

(b)

Fig. 21. Average fixed locations modified by through-dereference assignments. 


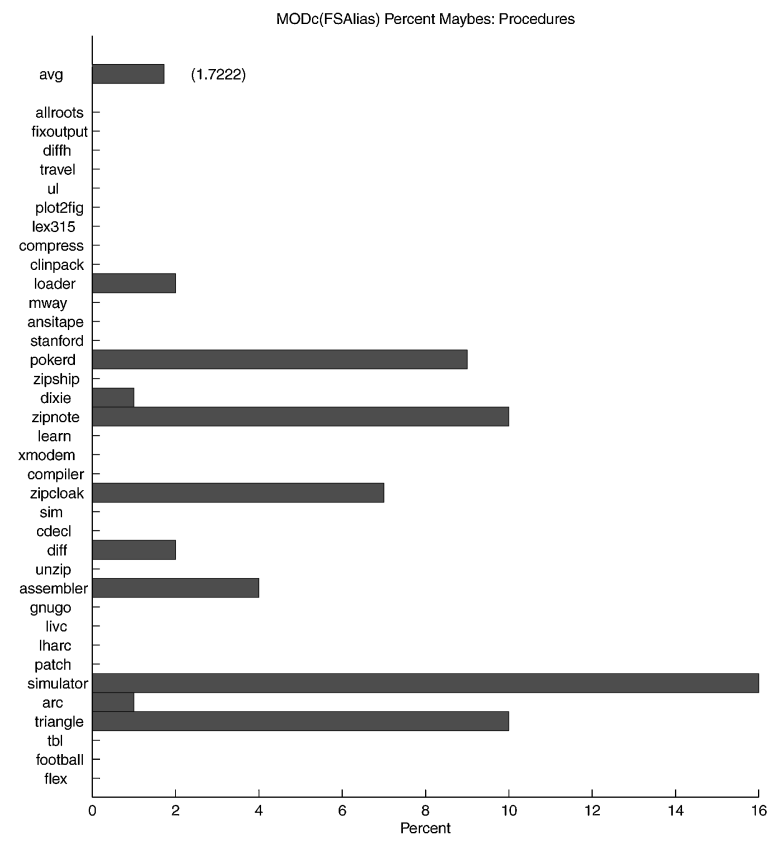

Fig. 22. Percentage of average number of modifications that are maybes.

aliases that do not occur on the same execution. We assume we are calculating a reverse-safe up to symbolic execution solution, and thus ignore the first type of imprecision. However, we can bound the second type of imprecision.

The equations in Section 3.1 can be extended to associate with each element of the $\mathrm{MOD}_{C}$ (FSAlias) solution either yes or maybe so that the part of the solution associated with yes is reverse-safe up to symbolic execution and the entire solution (i.e., the solution ignoring the yes/maybe information) is safe. Appendix B extends the equations in Section 3.1 in this manner. The safe solution implied by the modified equations is identical to the solution implied by the original equations.

The equations in Appendix B allow a bound on the second type of imprecision to be computed. All $\mathrm{MOD}_{C}$ (FSAlias) information not in the precise-up-tosymbolic-execution solution must be labeled with maybe. Thus, if $P$ percent of the solution is labeled with maybe then at most $P$ percent of the solution can be imprecise. Figure 22 is a bar chart of the percentage of $\operatorname{MOD}_{C}($ FSAlias $)$ labeled with maybe for results for procedures, whose data imply that a majority of solutions on this data set are precise up to symbolic execution as none of the solution is associated with maybe. Even for the programs that have some of their solution associated with maybe, that part of the solution is small (16\% maximum). The charts for through-dereference assignments and calls are similar in appearance to Figure 22 with averages of $4.1 \%$ and $2.4 \%$, respectively. Computing $\operatorname{MOD}_{C}($ FSAlias $)$ with yes/maybe information is more costly and could not be done for moria. Note that the precise up to symbolic execution solution at a program point is different from the must alias solution. For each alias in 


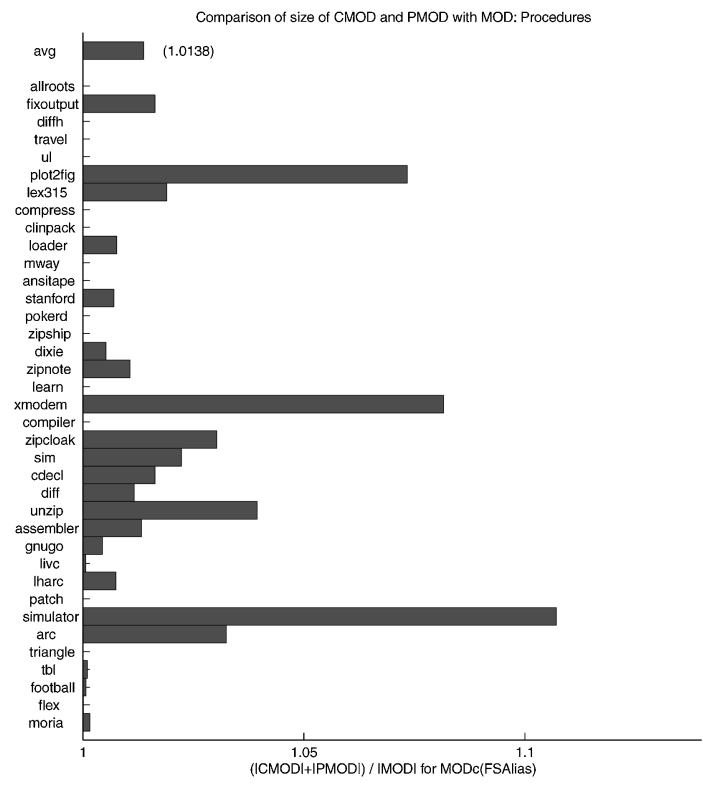

Fig. 23. Cost of keeping calling contexts.

the precise up to symbolic execution solution, there is some path in the ICFG which results in that alias. For an alias to be a must alias, it must exist on all paths to a program point.

\subsection{Cost of Keeping Calling Contexts}

The equations in Section 3.1 are context-sensitive in that they keep track of calling contexts in order to increase precision. This of course has a cost, and that cost is maintaining the same $\mathrm{MOD}_{C}$ information for different contexts. The ratio of the size of $C M O D$ plus the size of $P M O D$ to the size of $M O D$ indicates the percent of the $M O D$ solution that is represented redundantly under various calling contexts. Figure 23 gives this ratio for the programs in the data set for which the $\operatorname{MOD}_{C}$ (FSAlias) algorithm finds a solution. A ratio of 1.05 means that $5 \%$ of the solution is redundant. The average for these programs is around 1.01. Given the nature of Eqs. (3) and (4), this ratio should also be a good predictor of the extra time required to maintain the calling contexts.

\subsection{Effects of Counting}

Section 3.5 explains the issues in determining the size of the $M O D$ solution. Figure 24 compares the two methods of counting aggregates presented in that section for the $\operatorname{MOD}_{C}($ FSAlias $)$ solution at procedures. Let base be the number of modifications determined in the manner used throughout this paper (i.e., NoFields counting). The sum of base and extra is the number of modifications that would be determined by counting each part of an aggregate separately. What is important about this figure is that the numbers are very different depending on how modifications are counted. In order to compare work by different 


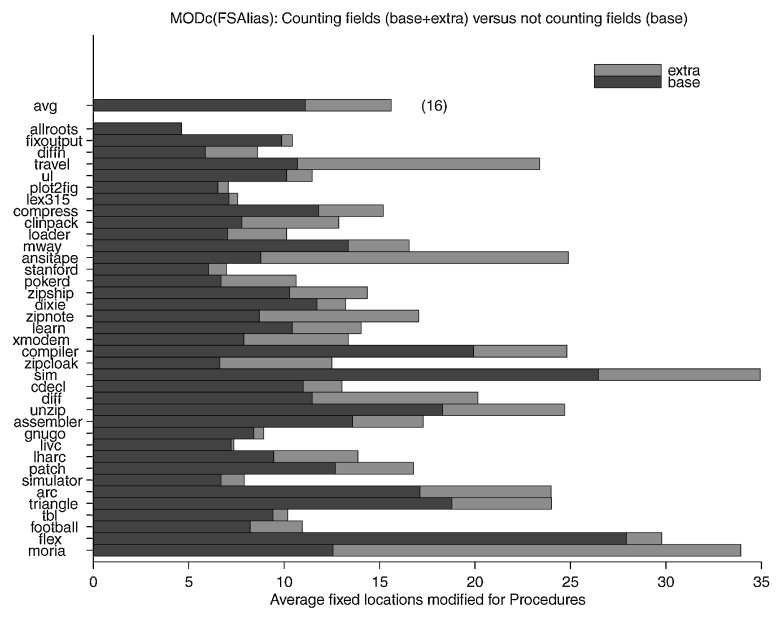

Fig. 24. Different methods of counting.

research groups, minimally the assumptions used while counting must be documented. It would be even more useful if the same method of counting was used by all research groups.

\subsection{Lines of Best Fit (Regressions)}

Regressions on the data in this paper are potentially interesting as they can indicate how various factors are related (e.g., how the size of the program correlates to the number of modifications). However, there are two major potential problems:

(1) the data set in this paper is too small to make strong conclusions, and

(2) the regressions might not be good.

The former point can only be dealt with by expanding the data set vastly (at least an order of magnitude). Regressions of many of the figures in this paper look reasonable. The regression in Figure 25(a) is one that appears good and is fairly typical. Given a line of best fit $y=m x+b$ for the data $\left\{\left(x_{i}, y_{i}\right)\right\}$ where $x_{i}$ is program size in ICFG nodes and $y_{i}$ is the program property being measured, an error factor $e_{i}$ can be determined for each $i$ such that $e_{i}=y_{i}-m x_{i}+b$. For example in Figure 16, $y_{i}$ is the number of fixed locations reported on average per procedure.

The regression line indicates that there is a slight correlation between program size and the number of modifications. An additional 886 ICFG nodes (i.e., program statements) increases the expected number of modifications by 1 at each call site. However, the error factor seems to be a more important factor than the program size. This indicates that while size plays a role there are other, probably more significant, factors involved. For the data in this paper, this is what the regressions typically indicate. The regression in Figure 25(a) is fairly good in that the plot of the errors (residuals) in Figure 25(b) yields a fairly random pattern (although the magnitude of the error might get larger 


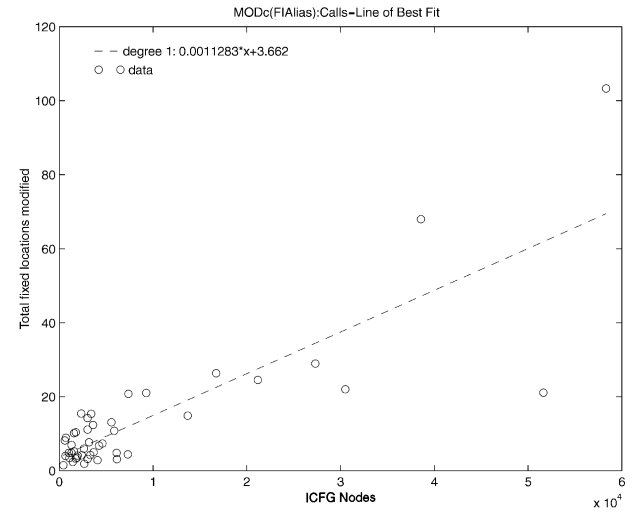

Line of best fit

(a)

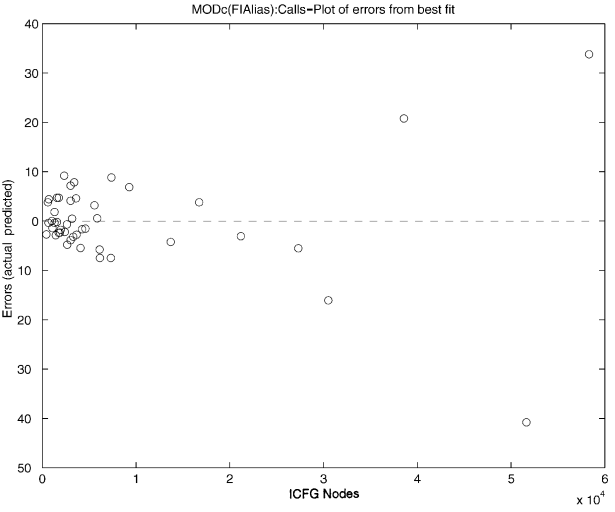

Errors in best fit

(b)

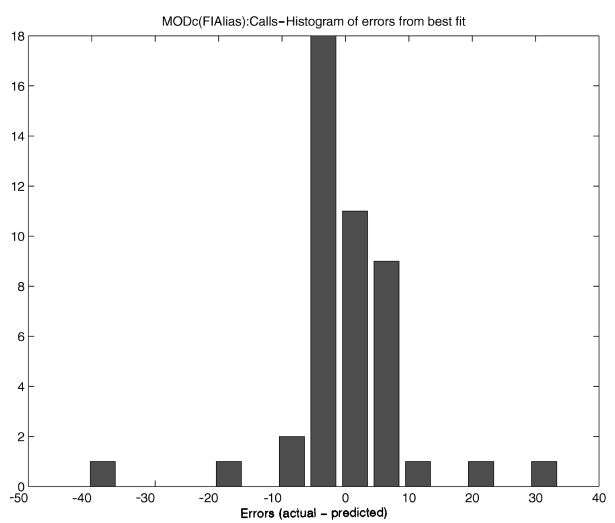

Histogram of errors in best fit

(c)

Fig. 25. A typical regression.

as program size gets larger) and the histogram of the errors in Figure 25(c) is roughly normal.

\subsection{Timing Results}

Timing results are reported for the analysis times of the $\mathrm{MOD}_{C}$ calculation, broken into its two passes, and for simple compilations of the programs in the data set using GNU's gcc compiler version 2.7.2 with no optimizations enabled and no linking. (All compile times reported in this section used this GNU compiler.) These numbers are as reported by the UNIX time utility, averaged over 5 executions. The notation $\operatorname{MOD}_{C}(\mathrm{FS})$ refers to the phase of the $\mathrm{MOD}_{C}($ FSAlias $)$ algorithm after the alias solution has been computed; similarly for $\mathrm{MOD}_{C}(\mathrm{FI})$. Thus, these times do not include the alias analysis times, but are simply the time taken to calculate the $\mathrm{MOD}_{C}$ solution given the alias solution. The total 


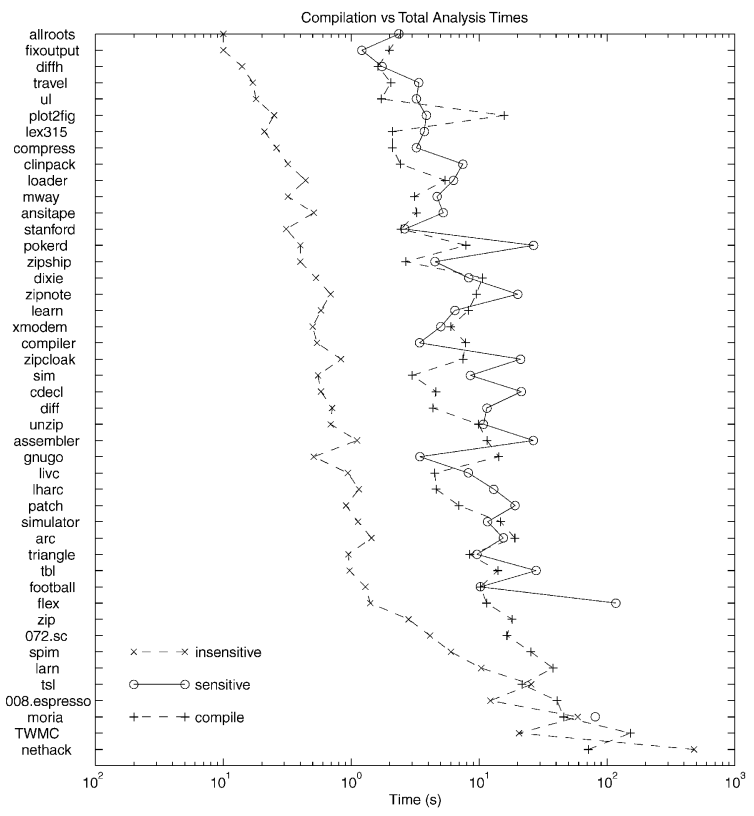

(a)

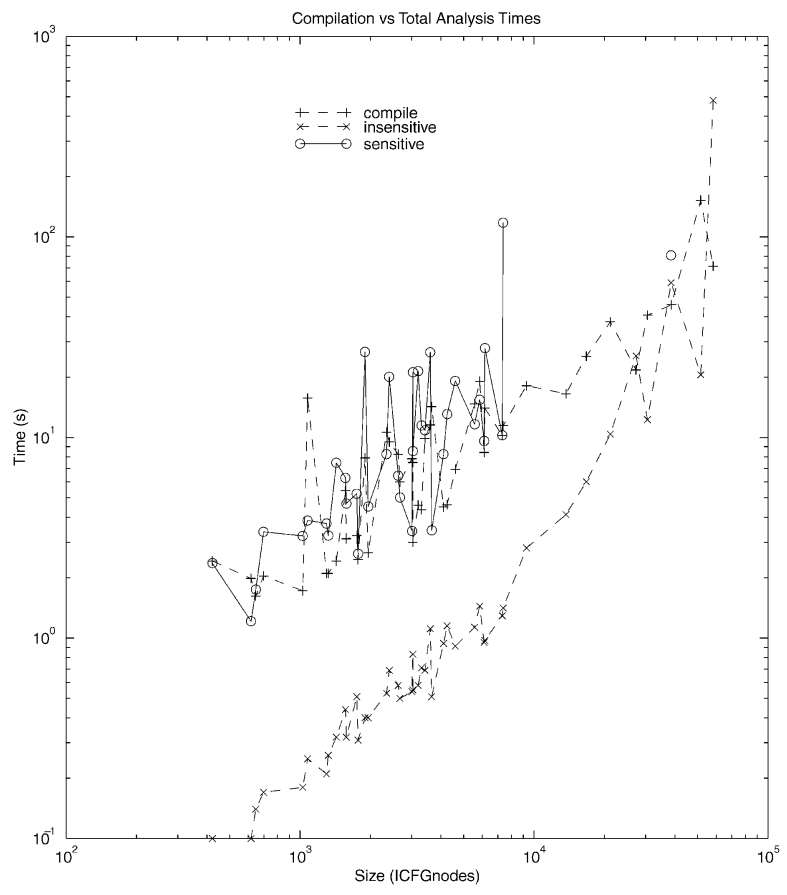

(b)

Fig. 26. $\operatorname{MOD}_{C}($ FSAlias $)$ time, $\operatorname{MOD}_{C}$ (FIAlias) time, and compile time. ACM Transactions on Programming Languages and Systems, Vol. 23, No. 1, March 2001. 


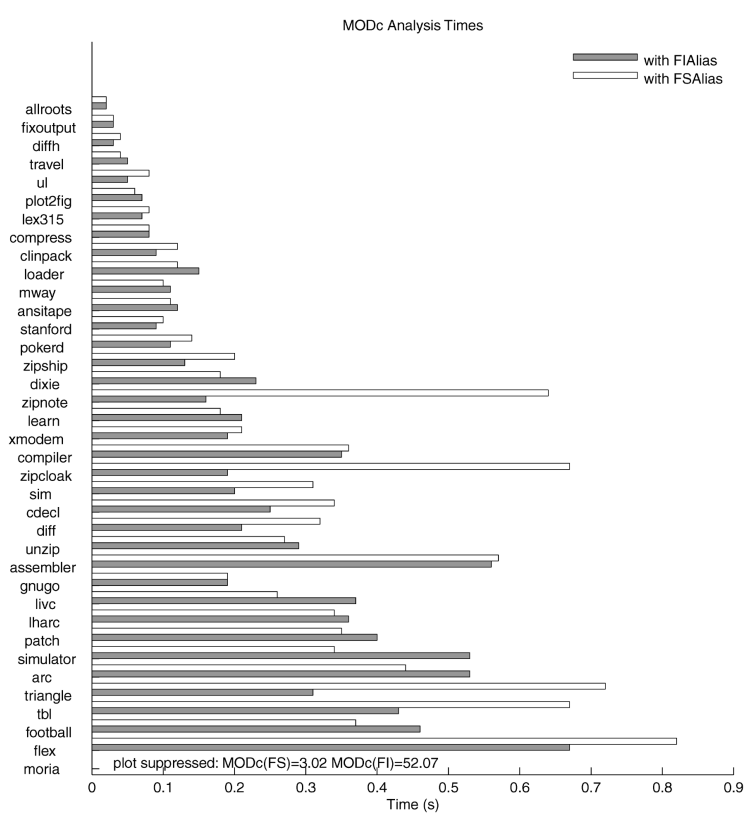

Fig. 27. $\mathrm{MOD}_{C}(\mathrm{FS})$ and $\mathrm{MOD}_{C}(\mathrm{FI})$ analysis times.

analysis time for, say, the flow-/context-sensitive analysis is the sum of the FSAlias time and the $\mathrm{MOD}_{C}(\mathrm{FS})$ time. The $\mathrm{MOD}_{C}$ analysis time is dominated by the alias calculation.

Figure 26(a) contrasts the $\operatorname{MOD}_{C}($ FSAlias $)$ and $\operatorname{MOD}_{C}($ FIAlias $)$ analysis times versus the compile times for the data programs, using a logarithmic scale for the time axis ( $x$-axis). Figure 26(b) plots the same data, but uses the program sizes (in ICFG nodes) as the $x$-axis showing the times on log scale on the $y$-axis. These figures demonstrate the dramatic difference between the sensitive and insensitive analyses, showing at least an order of magnitude difference between the two. The good news is that in most cases the FSAlias analysis time is comparable to the compile time. This is an important feature for any analysis destined for compiler optimization.

Figure 27 shows the times for the $\mathrm{MOD}_{C}(\mathrm{FS})$ and $\mathrm{MOD}_{C}(\mathrm{FI})$ phases of the $\mathrm{MOD}_{C}$ calculation. $\mathrm{MOD}_{C}(\mathrm{FS})$ and $\mathrm{MOD}_{C}(\mathrm{FI})$ are both very fast, and are comparably fast, except in the case of moria. Notice that seemingly large differences are not large because the scale of the time axis is so small. For some of the programs, differences between $\mathrm{MOD}_{C}(\mathrm{FS})$ and $\mathrm{MOD}_{C}(\mathrm{FI})$ for the same program seem explainable by possible noise in our measurements. The only explanation so far for moria is that the FIAlias solution is sufficiently imprecise to cause the second pass of the $\mathrm{MOD}_{C}$ algorithm to do significantly more work. Table III in Appendix $\mathrm{C}$ shows the raw data from which these figures are constructed.

\subsection{Measurement of Memory Needed}

The maximum memory needed by the two $\mathrm{MOD}_{C}$ analyses was measured empirically by an external process while each analysis was running on each program 


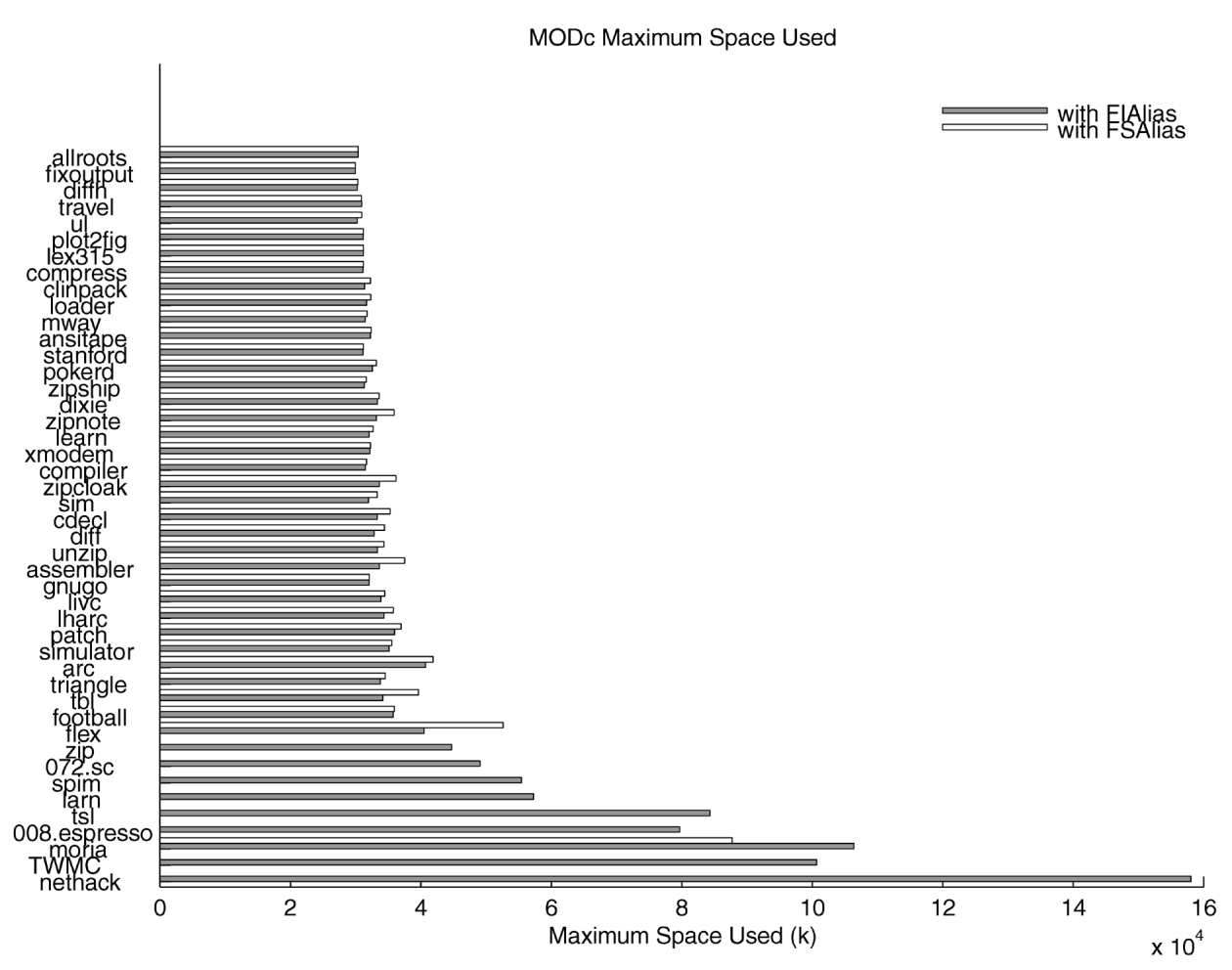

(a)

Fig. 28. Memory usage of $\operatorname{MOD}_{C}$ (FSAlias) and $\operatorname{MOD}_{C}$ (FIAlias).

in the data set. The memory needs of each algorithm include (i) a fixed-size of overhead space for algorithm data structures, (ii) the program representation, whose size varies linearly with program size and was the same for both analyses, and (iii) the analysis results. The maximum memory reported as used for each program during the two phases of $\mathrm{MOD}_{C}$ analysis in Figure 28 includes all three of these factors.

Figure 28(a) shows the absolute space needed, which varies for $\mathrm{MOD}_{C}($ FIAlias $)$ from about $40 \mathrm{MB}$ to almost $160 \mathrm{MB}$. The maximum space needed for $\mathrm{MOD}_{C}($ FSAlias $)$, which runs out of virtual memory on the large programs, was about $90 \mathrm{MB}$ on moria. Note that the ratio of program size between flex and moria (as measured by number of ICFG nodes-see Table I) is about 1:5.5 whereas the difference in maximum space needed for $\operatorname{MOD}_{C}($ FSAlias $)$ is only about $50 \%$. The maximum space needs of $\operatorname{MOD}_{C}($ FIAlias $)$ seem to be growing precipitously; however, another way to view these results is shown in Figure 28(b), where the maximum space needed by each analysis is normalized by the number of ICFG nodes (i.e., approximately the number of $\mathrm{C}$ statements in the program). Here it is clear that the memory needs of $\mathrm{MOD}_{C}$ (FIAlias) per statement of code are fairly stable for the larger programs (i.e., more than $20 \mathrm{~K}$ LOC). Since no attempt has been made to optimize for memory usage in either 


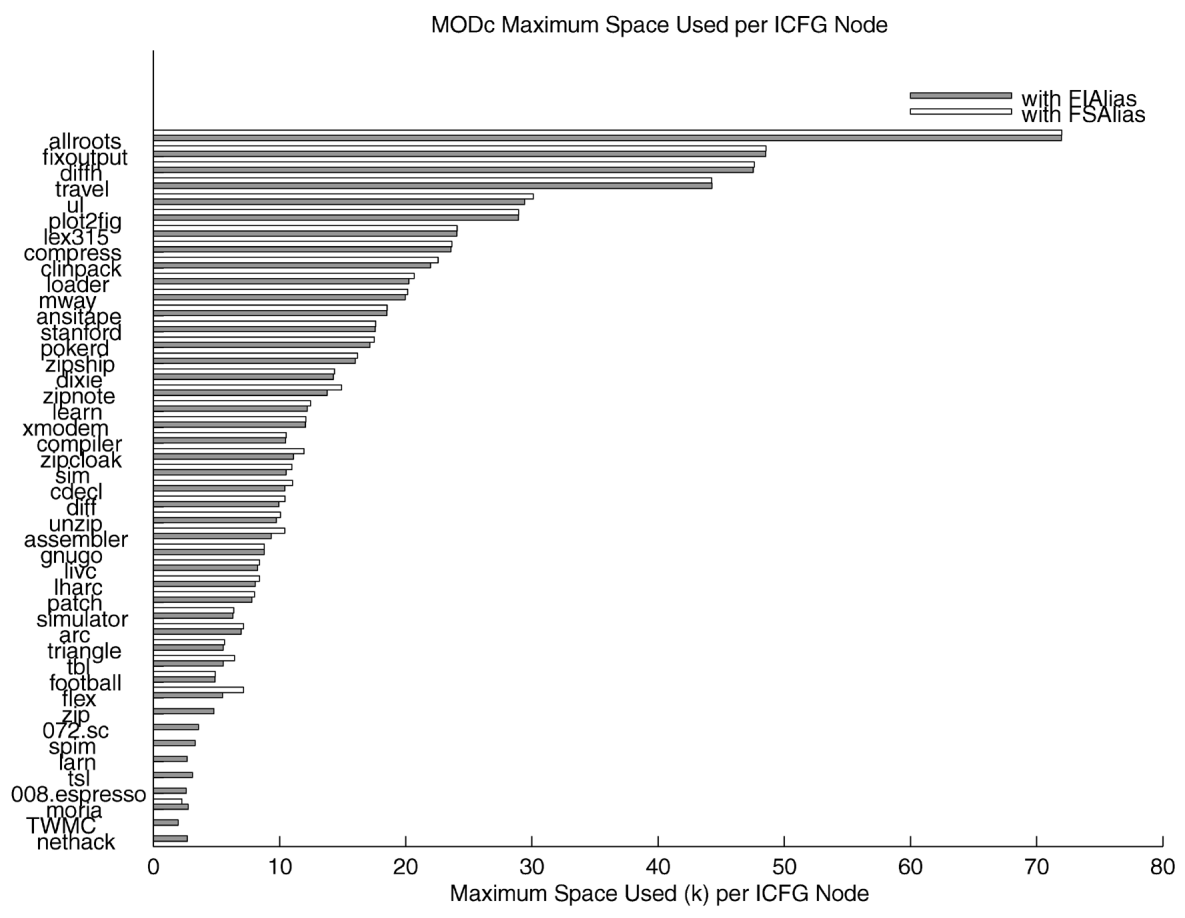

(b)

Fig. 28. Continued.

analysis, these measurements are a "first cut" at what memory needs limit these analyses.

\section{RELATED WORK}

Interprocedural Side-Effects Analysis. Interprocedural modification side effects were first handled by Allen for acyclic call multigraphs in FORTRAN programs [Allen 1974; Spillman 1971]. Later, Barth [1978] explored the use of relations to capture side effects in recursive programs. Banning [1979] first accomplished the decomposition of the MOD problem for FORTRAN (and other languages where aliasing is imposed only by call-by-reference parameter passing); he separated out two flow-insensitive calculations on the call multigraph: one for side effects and a separate one for aliases. The interprocedural sideeffects problem for FORTRAN is flow-insensitive, but context-sensitive. Cooper and Kennedy [Cooper 1985; Cooper and Kennedy 1987; 1988] further decomposed the problem into side effects on global variables and side effects accomplished through parameter passing. Burke showed that these two subproblems on globals and formals can be solved by a similar problem decomposition [Burke 1990]. All of this work targeted the programming model of FORTRAN77, a language without pointers. Choi, Burke, and Carini mention an interprocedural modification side-effects algorithm for languages with pointers based on their 
flow-sensitive pointer-aliasing analysis technique [Choi et al. 1993; Marlowe et al. 1993]; it is difficult to compare their work to this work, because they give no description of their algorithm and offer no implementation results.

Another approach to side-effect analysis is to perform an interprocedural pointer-aliasing algorithm and then identify all variables experiencing side effects at indirect stores through a pointer (i.e., at through-dereference statements) using the aliases found [Emami et al. 1994; Ghiya and Hendren 1998; Hind and Pioli 1998; Ruf 1995; Shapiro and Horwitz 1997a; Zhang et al. 1996; 1998]. This is often used as an empirical test of the precision of the alias solution obtained.

Related Analyses. Related interprocedural analyses include compile-time interprocedural program slicing [Atkinson and Griswold 1996; 1998; Gallagher and Lyle 1991; Gupta and Soffa 1996; Harrold and Ci 1998; Horwitz et al. 1990; Larsen and Harrold 1996; Ottenstein and Ottenstein 1984; Reps and Rosay 1995; Sinha et al. 1999; Tip 1996; Tip et al. 1996; Tonella et al. 1997; Venkatesh 1991; Weiser 1984], interprocedural def-use associations [Chatterjee and Ryder 1999; Ghiya and Hendren 1998; Harrold and Soffa 1994; Pande et al. 1994], and demand analyses [Duesterwald et al. 1995; 1996; Horwitz et al. 1995]. Slicing determines the data- and control-dependent parts of a program which correspond to a particular computation. Def-use associations trace value flow on static paths in a program; they are useful for various machine-independent optimizations and data-flow testing methods. Demand data-flow analysis seeks to efficiently answer queries about individual dataflow facts at a program point; a partial calculation is performed to derive the data-flow information, rather than a whole program analysis.

Interprocedural distributive finite subset problems can be solved using a graph reachability technique on an "exploded" call graph of the program [Reps et al. 1995]. Capture of calling context is not an issue here since the problems being solved are of a form such that reachability in each procedure can be analyzed once for each parameter, regardless of calling context. The solution at a call site is obtained by using the parameter-binding functions to identify incoming information with outgoing information at the corresponding return site. These relations between incoming and outgoing information are then memoized to avoid re-analysis. The underlying ideas of this analysis are related to notions expressed in the conditional analysis for aliasing due to single-level pointers [Landi and Ryder 1991]. Several solutions to MOD $_{C}$ with different flow-insensitive, context-insensitive points-to approximation algorithms have been obtained by this method, since using program-wide aliases yields an approximate problem for $\mathrm{MOD}_{C}$ that yields the same side effects regardless of calling context [Shapiro and Horwitz 1997a]. This Horwitz and Shapiro study shares the philosophy of the empirical results presented here, in that the effects of pointer aliasing on applications are reported. However, there are no flow- and/or context-sensitive analyses performed, and direct comparison with $\operatorname{MOD}_{C}($ FIAlias $)$ is difficult, since only a flow-/context-insensitive $M O D(P)$ is defined with no per-statement side effects, and indirect side effects to structure fields and union members are not distinguished. 
Ruf [1995] compared the effect of context sensitivity (or its lack) on a flowsensitive points-to algorithm. Most of his reported data is with respect to the difference in precision of the points-to solution, with and without context information. Although his results were based on a possibly nonrepresentative benchmark suite (a fact observed by him), they indicated that no precision improvement was observed for his flow-sensitive algorithm on these programs by adding context sensitivity. For many reasons this study is difficult to compare with the results presented here. First, his VDG program representation is incomparable to the ICFG, not being a statement-level representation. Secondly, several independent structure field references may be merged, so that data on "average" number of side effects at a write statement may not be comparable to data at through-dereference statements. Thirdly, there is no flow-insensitive method in his paper.

Newer studies of the affect of flow sensitivity on points-to analysis [Hind and Pioli 1998; 2000] carefully track the performance of several flow-insensitive algorithms versus that of a flow-sensitive algorithm [Burke et al. 1994; 1997; Choi et al. 1993; Hind et al. 1999; Marlowe et al. 1993]. Comprehensive measurements of comparative precision at through-dereference statements (as well as precision on dereferenced reads), algorithm timings, and memory usage are reported. These experiments on this data set showed that the precision of their flow-insensitive analysis was identical to that of their flow-sensitive analysis on 12 of their 21 benchmark programs [Hind and Pioli 1998]. Further work [Pioli 1999] studies the affect of analysis precision on constant propagation. It is difficult to compare Hind and Pioli's results with those reported here, since all of their algorithms are context-insensitive and a different alias representation is used, which may alter the fixed location counts.

Pointer May_Alias Algorithms. This paper discusses a schema for finding side effects in $\mathrm{C}$ codes that is parameterized by the type of pointer-aliasing technique used. Since the focus of this paper is the $\mathrm{MOD}_{C}$ schema, the recent work in pointer analysis is merely summarized here. Recently, there have been many investigations of pointer-aliasing algorithms which vary in cost and precision. Several concentrate on aliases in heap storage [Chase et al. 1990; Deutsch 1994; Ghiya and Hendren 1996a; 1996b; Horwitz et al. 1989; Hendren and Nicolau 1990; Jones and Muchnick 1982a; Larus and Hilfinger 1988; Sagiv et al. 1998]. Others calculate flow-insensitive aliases with recent emphasis on algorithm scalability on very large programs (i.e., one million lines of code) [Andersen 1994; Burke et al. 1994; Coutant 1986; Das 2000; Fahndrich et al. 2000; Foster et al. 2000; Guarna 1988; Hind and Pioli 1998; Liang and Harrold 1999; Rountev and Chandra 2000; Shapiro and Horwitz 1997b; Steensgaard 1996b; Weihl 1980; Zhang et al. 1996]. There are flow-sensitive techniques as well which calculate program-point-specific aliases [Choi et al. 1993; Cooper 1989; Chatterjee et al. 1999; Emami et al. 1994; Ghiya and Hendren 1998; Harrison and Ammarguellat 1990; Landi and Ryder 1992; Marlowe et al. 1993; Ruf 1995; Sagiv et al. 1990; Wilson and Lam 1995]. Other work concentrates on aliases in higher-order functional languages [Deutsch 1990; Neirynck et al. 1987]. SSA-form is used to transform the program in a way that effectively 
adds "adjustable" flow sensitivity to a flow-insensitive pointer-alias analysis in [Hasti and Horwitz 1998]; no empirical results are given, and it is not clear if this technique is scalable. Newer work calculates pointer aliasing in programs with explicit concurrency achieved with co-begin, co-end constructs [Rugina and Rinard 1999]. Another new paper offers a framework for normalizing the representation of structure fields, for use in determining aliasing in the presence of unions and casting [Yong et al. 1999].

\section{OBSERVATIONS}

Recall that the empirical experiments refer to two specific implemented $\mathrm{MOD}_{C}$ algorithms, $\mathrm{MOD}_{C}($ FSAlias $)$ and $\operatorname{MOD}_{C}$ (FIAlias). These observations are obtained from examination of these results and are specific to them. The obvious conclusion of the empirical results is that $\operatorname{MOD}_{C}($ FSAlias $)$ yields significantly more precise solutions at far greater computation cost. Nevertheless, this is a complex and interesting trade-off.

\subsection{Flow-/Context-Sensitive Analysis}

The flow-/context-sensitive data-flow analysis presented here is capable of providing very accurate results for small, real-world applications (10K LOC). As expensive as it is, the cost of sensitive analysis is still not prohibitive for a large subset of the data programs, being on the order of the time to compile the program. Thus, the $\operatorname{MOD}_{C}$ (FSAlias) algorithm achieves scalability up to a certain point. Recall that previously published results for flow-/context-sensitive side-effect analysis were only up to 4700 lines of code. In particular, substantially larger programs that do not use certain program constructs heavily can be analyzed; moria and zip vividly show the effects on the $\mathrm{MOD}_{C}$ analyses presented here of heavy use of (large) recursive data structures. This level of scalability is rather surprising for a program-point-specific analysis. Further, note that users of software engineering tools, such as data-flow testers or offline program-understanding databases which gather def-use information about a large program in order to query it later, may be willing to accept analysis costs of several times that of the compilation time and/or large memory needs. Nevertheless, it seems apparent that flow-/context-sensitive analysis is not going to scale to the next order of magnitude without a major innovation; whole-program flow-/context-sensitive analysis of large systems seems unattainable.

\subsection{Flow-/Context-Insensitive Analysis}

The flow-/context-insensitive analysis presented here is a very fast and scalable analysis. Whole-program analysis of large software, such as today's commercial applications, seems feasible. The loss of precision is a strong concern, however. Most applications of the modification side-effects solution need quite precise results (e.g., data-flow-based testing). Nevertheless, it is interesting that the flow-/context-insensitive solutions are much more precise than the worstcase estimate, meaning that there is still significant gain to be had from using this inexpensive analysis. Software engineering tools such as smart semantic browsers which trace approximate def-use information or debuggers which use 
run-time traces augmented by compile-time knowledge are possible consumers of insensitive side-effect information. So, flow-insensitive analysis can be very effective, being inexpensive and acceptably accurate for certain applications.

\subsection{Comparison of Sensitivity}

One claim being disputed in the analysis community is that flow-/contextsensitive analysis will obtain much better precision than flow-/contextinsensitive analysis on important problems, such as modification side effects. The empirical results confirm the belief that sensitivity provides discernably increased precision in the solution obtained; for program transformation or validation applications, this accuracy may be required.

\subsection{Where to Now?}

This study raises three topics for further exploration. The first is how to incorporate flow sensitivity into analysis of very large programs. Zhang et al. [1996; 1998 report on a program decomposition strategy in which the alias relation induces a partitioning of the assignment statements involving pointer variables. This in turn can be used to decompose the program into sections for which analyses of differing precision and cost can be applied. Initial experiments targeted recursive data structures as subjects for a flow-/context-insensitive alias analysis with appealing results. Reorganization and redesign of flow-/contextsensitive analysis to reduce memory costs of points-to analysis in $\mathrm{C}^{++}$and Java is the focus of [Chatterjee 1999; Chatterjee et al. 1999]; preliminary empirical findings are encouraging. More recent work investigates program fragment analysis in which an initial, coarse-grained, whole-program analysis is followed by an analysis of a fragment or module, which obtains more precise data-flow information on that fragment [Rountev et al. 1999]. This offers the promise of a focused analysis that yields greater precision where it is needed.

The second topic is how to make flow-/context-insensitive analysis more effective without increasing the cost. An interesting idea stems from the observation that safe analyses produce supersets of the precise solution. The intersection of the solutions generated by different, safe analyses for the same problem must also be safe, and may be closer to the precise solution. Recently, Shapiro and Horwitz [1979b] used this idea with several flow-/context-insensitive points-to analysis algorithms. This approach needs more exploratory experimentation.

The final topic is to discern more fully the kind of program construct and programming style that foils data-flow analysis. Perhaps the availability of precise, flow-/context-sensitive data-flow analysis would be sufficient motivation to change programming practice, language design, and programmers' habits. For instance, references in Java are a restricted form of pointers, and might be substantially easier to deal with under static analysis than C's general-purpose pointers.

\section{CONCLUSION}

This is the first interprocedural modification side-effects analysis for $\mathrm{C}\left(\mathrm{MOD}_{C}\right)$ that obtains reasonable precision on programs with general-purpose pointer 
usage. The algorithm schema is parameterized by choice of pointer-aliasing method used as the first pass. Two $\mathrm{MOD}_{C}$ algorithms at opposite ends of the spectrum in terms of flow and context sensitivity were empirically profiled, with data collected for key statements (i.e., through-dereference assignments and calls) as well as for procedures (i.e., $\operatorname{MOD}(\mathrm{P})$ ). This is the first empirical comparative study of the effects of both flow and context sensitivity in the context of an important data-flow problem. It is especially significant that the utility of the data-flow solution obtained is studied in an application context, because the hypothesis is that different applications will select different trade-offs in cost versus precision. A significant precision advantage was established for the flow/context-sensitive side-effects analysis over that of the flow-/context-insensitive analysis; but, this performance was at a severe cost in analysis execution time usually of at least an order of magnitude. Maximum memory usage was tracked for these types of analyses, but no meaningful comparison can be made with regard to memory usage until analyses have been optimized to save space.

\section{APPENDIX}

\section{A. COMPARISON WITH THE MOD DECOMPOSITION FOR FORTRAN}

The decomposition of the MOD problem for $\mathrm{C}$ in Figure 10 is similar in structure to the original decomposition for FORTRAN by Banning [1979], in the sense that both calculate local side effects in each procedure first, and then set up data-flow equations on call graphs to compute procedure-level side effects (i.e., a flow-insensitive interprocedural calculation).

The two decompositions are also similar in what is included in the MOD sets. In FORTRAN programs, variables are the only fixed locations, and therefore various MOD sets in the decomposition for FORTRAN include just variable names. In $\mathrm{C}$, pointers and dynamic allocation are allowed.

The two decompositions differ in their treatment of aliases. In the FORTRAN decomposition, aliases are computed at procedure calls. This is possible because for FORTRAN programs, only procedure calls can create aliases, and aliases created by a call hold throughout execution of the procedure being called. In the MOD decomposition in Figure 10, aliases are computed at pointer assignments and procedure calls (i.e., at program points), because aliases vary intraprocedurally. An alias at a program point is associated with a reaching alias for the procedure containing that program point. These reaching aliases differentiate side effects caused by different calls of the same procedure.

The MOD problem for FORTRAN was further decomposed by Cooper [1985] and Cooper and Kennedy [1987; 1988] into two subproblems, one on global variables and another on reference formals. The first subproblem is provably rapid [Marlowe and Ryder 1990b] while the second one is not. Similarly, for the C language, side effects on global fixed locations (including heap storage creation site names) can be separated from side effects on locals and non-visibles.

This can be done by introducing a new set $\operatorname{CondIMOD}^{+}(P, R A)$ to the decomposition of Figure 10. This new set is the set of fixed locations either modified directly in procedure $P$ or modified as non-visibles in procedures called by $P$, considering only aliases in $P$ that are conditioned on $R A$. The non-visibles modified 
by procedures called in $P$ are local variables of either $P$ or other procedures that have called $P$, directly or indirectly. The system of data-flow equations for CondIMOD $^{+}$sets is as follows, where $b_{\text {call }_{Q}}$ is the function $b_{\text {call }}$ in Section 3.1 restricted to mapping of non-visibles in $Q$ to either locals or non-visibles in $P$.

$$
\begin{aligned}
& \operatorname{CondIMOD}^{+}(P, R A)= \\
& \operatorname{CondIMOD}(P, R A) \bigcup \bigcup\left(b_{\text {call }_{Q}}^{\prime}\left(\operatorname{CondIMOD}^{+}\left(Q, R A^{\prime}\right)\right)\right) \\
& \text { call }_{Q} \in P \text { and } \\
& R A^{\prime} \in \text { contexts_of }\left(\text { call }_{Q}, R A\right)
\end{aligned}
$$

After obtaining $\operatorname{CondIMOD}^{+}(P, R A)$ solutions, the PMOD sets can be computed by solving the following data-flow equations.

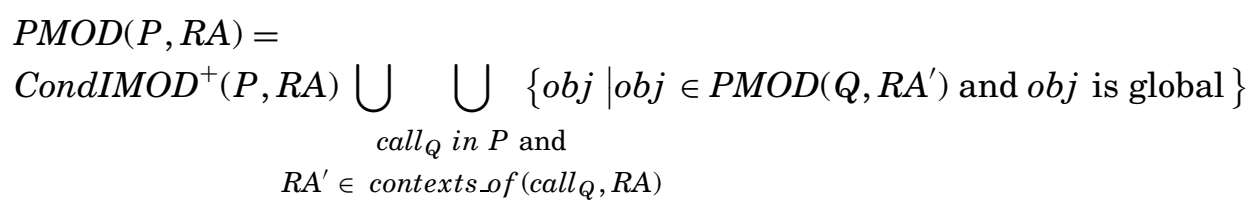

Figure 29 compares various MOD sets defined in this MOD decomposition for $\mathrm{C}$ and those in the decomposition for FORTRAN as presented in Cooper and Kennedy [1987].

\section{B. CALCULATION OF MOD WITH maybes}

In order to incorporate maybe information from the alias solution into the MOD solution, the following definitions are needed:

- $\mathcal{L}_{1}$ is the lattice ( $\{$ maybe, yes $\left.\}, \sqsubseteq_{1}, \sqcup_{1}, \cap_{1}, \top_{1}, \perp_{1}\right)$ implied by the relation maybe $\sqsubseteq_{1}$ yes.

- $F$ is the set of fixed locations of the program being analyzed.

- $\mathcal{L}$ is $(S, \sqsubseteq, \sqcup, \sqcap, \top, \perp)$

$-S=\operatorname{powerset}(\boldsymbol{F} \times\{$ maybe, yes $\})$

$\multimap a \sqsubseteq b$ iff $\left[(\forall s)\left[s=\left(f, d_{1}\right) \in a\right] \Rightarrow\left[\left(\exists d_{2}\right)\right.\right.$ such that $\left(f, d_{2}\right) \in b$ and $\left.\left.d_{1} \sqsubseteq_{1} d_{2}\right]\right]$

$$
-a \sqcup b=\bigcup_{f \in F}\left\{\begin{array}{ll}
\{(f, d)\} & \begin{array}{l}
{\left[\left(\exists d_{1}\right) \text { such that }\left(f, d_{1}\right) \in a\right] \wedge} \\
{\left[\left(\exists d_{2}\right) \text { such that }\left(f, d_{2}\right) \in b\right] \wedge} \\
d=d_{1} \sqcup_{1} d_{2}
\end{array} \\
\{(f, d)\} & \left\{\begin{array}{l}
(f, d) \in a \wedge \\
{\left[\left(\nexists d_{1}\right) \text { such that }\left(f, d_{1}\right) \in b\right]} \\
{\left[\left(\nexists d_{1}\right) \text { such that }\left(f, d_{1}\right) \in a\right]}
\end{array}\right. \\
\emptyset & \text { otherwise }
\end{array}\right)
$$

ACM Transactions on Programming Languages and Systems, Vol. 23, No. 1, March 2001. 


\begin{tabular}{|c|c|}
\hline $\begin{array}{l}\text { MOD Decomposition for } \\
\text { FORTRAN [Cooper and Kennedy1987] } \\
s \text { is a statement. } \\
P \text { is a procedure. }\end{array}$ & $\begin{array}{l}\text { MOD Decomposition for } \mathbf{C} \\
n \text { is either an assignment or a call. } \\
P \text { is a procedure. } \\
R A \text { is a reaching alias. }\end{array}$ \\
\hline $\begin{array}{l}M O D(s) \\
\text { the set of variables modified by an } \\
\text { execution of } s, \text { excluding any } \\
\text { procedure calls in } s\end{array}$ & $\begin{array}{l}\text { CondLMOD }(n, R A) \\
n \text { is an assignment. } \\
\text { the set of fixed locations modified by an execution of } \\
n \text { considering aliases that are associated with } R A \\
\text { and hold on entry } n .\end{array}$ \\
\hline $\begin{array}{l}I M O D(P) \\
\text { the set of variables modified by an } \\
\text { invocation of } P \text {, excluding any } \\
\text { procedure calls in } P\end{array}$ & $\begin{array}{l}\text { CondIMOD }(P, R A) \\
\text { the set of fixed locations modified by an invocation of } \\
P \text {, considering only assignments in } P \text { and aliases } \\
\text { associated with } R A \text { in } P\end{array}$ \\
\hline $\begin{array}{l}I M O D^{+}(P) \\
\text { the set of variables either modified } \\
\text { directly in } P \text { or modified as } \\
\text { reference formals in procedures called } \\
\text { in } P\end{array}$ & $\begin{array}{l}\text { CondIMOD }{ }^{+}(P, R A) \\
\text { the set of fixed locations either modified directly in } \\
P \text { or modified as non-visibles in procedures called by } P \text {, } \\
\text { considering only aliases associated with } R A \text { in } P\end{array}$ \\
\hline $\begin{array}{l}G M O D(P) \\
\text { the set of variables modified by an } \\
\text { invocation of } P \text {, including procedure } \\
\text { calls in } P \text { and ignoring any aliases } \\
\text { in } P\end{array}$ & $\begin{array}{l}P M O D(P, R A) \\
\text { the set of fixed locations modified by an invocation of } \\
P \text {, considering both assignments and procedure calls in } P \text {, } \\
\text { and aliases associated with } R A \text { in } P\end{array}$ \\
\hline $\begin{array}{l}D M O D(s) \\
\text { the set of variables modified by an } \\
\text { execution of } s, \text { including procedure } \\
\text { calls in } s \text { and ignoring any aliases } \\
\text { in the procedure containing } s\end{array}$ & $\begin{array}{l}C M O D(n, R A) \\
n \text { is either an assignment or a call. } \\
\text { the set of fixed locations modified by an execution of } \\
n \text {, considering aliases that are associated with } R A \\
\text { and hold on entry } n \text {, and parameter bindings if } n \text { is a call }\end{array}$ \\
\hline $\begin{array}{l}M O D(s) \\
\text { the set of variables modified by an } \\
\text { an execution of } s, \text { considering all } \\
\text { aliases in the procedure containing } s\end{array}$ & $\begin{array}{l}M O D(n) \\
n \text { is either an assignment or a call. } \\
\text { the set of fixed locations modified by an execution of } \\
n, \text { considering all possible aliases true on entry } n \\
\text { in the procedure containing } n\end{array}$ \\
\hline
\end{tabular}

Fig. 29. Comparison of MOD decompositions for FORTRAN and C.

$-\sqcap$ is analogous to $\sqcup$ but is not needed

$-\top=F \times\{$ yes $\}$

$-\perp=\emptyset$

- DIRMOD, Predecessors, and $b_{\text {call }_{Q}}$ as in Section 3.

- For all program points $n$ and all reaching aliases $R A$

$-(\langle x, y\rangle, y e s) \in A l i a s^{\prime}(n, R A)$ if $x$ and $y$ are definitely aliases on some path to $n$ given $R A$ reaches the entry of the procedure containing $n$. This is simply a guarantee that $\langle x, y\rangle$ is in the precise up to symbolic execution alias solution. Note that the precise up to symbolic execution solution at a program point is different from the must alias solution. For each alias in the precise up to symbolic execution solution, there is some path in the ICFG which results in that alias. For an alias to be a must alias, it must exist on all paths to a program point.

$-(\langle x, y\rangle$, maybe $) \in \operatorname{Alias}^{\prime}(n, R A)$ if $x$ and $y$ may be aliases on some path to $n$ given $R A$ reaches the entry of the procedure containing $n$ and the alias algorithm assumes it is for safety. 
- For all program points $n,(\langle x, x\rangle, y e s) \in \operatorname{Alias}^{\prime}(n, \phi)$ where $\langle x, x\rangle$ is any trivial, reflexive alias.

- contexts_of' is identical to contexts_of in Section 3.1 with yes or maybe associated with each alias as above.

The MOD problem can be decomposed when yes and maybe are to be associated with the solution in a manner similar to that in Figure 10.

First, notice that $o b j \in \operatorname{CondLMOD}(n, R A)$ should be associated with yes (maybe) iff the alias responsible for it is associated with yes (maybe).

$$
\begin{aligned}
& \operatorname{CondLMOD}^{\prime}(n, R A)= \\
& \bigcup_{\text {pred } \in \text { Predecessors }(n)}\left\{\begin{array}{l|l}
\left(o b j_{1}, b\right) & \begin{array}{l}
o b j_{2}=\operatorname{DIRMOD}(n) \text { and } \\
\left(\left\langle o b j_{1}, o b j_{2}\right\rangle, b\right) \in A l i a s^{\prime}(\text { pred }, R A) \\
\text { and obj is a fixed location }
\end{array}
\end{array}\right\}
\end{aligned}
$$

For a procedure $P$ and reaching alias $R A, \operatorname{CondIMOD}^{\prime}(P, R A)$ contains the fixed locations modified by assignments in procedure $P$ :

$$
n \text { an assignment in } P
$$

A fixed location in $P M O D^{\prime}(P, R A)$ is definitely modified if it is definitely modified (associated with yes) in a called procedure and the alias at the call site which triggers that modification is also associated with yes:

$$
\begin{aligned}
& \operatorname{PMOD}^{\prime}(P, R A)=\operatorname{CondIMOD}^{\prime}(P, R A) \cup \\
& \bigcup_{\begin{array}{c}
\text { call } \text { in } P \text { and } \\
\left(R A^{\prime}, b^{\prime}\right) \in \text { contexts_of }
\end{array}}\left\{\begin{array}{l|l}
(o b j, b) & \begin{array}{l}
\left.\left(o b j^{\prime}, b^{\prime \prime}\right) \in P M O D^{\prime}, R, R A^{\prime}\right) \wedge \\
o b j \in b_{\text {call }_{Q}}\left(\left\{o b j^{\prime}\right\}\right) \wedge b=b^{\prime} \sqcap_{1} b^{\prime \prime}
\end{array}
\end{array}\right\}
\end{aligned}
$$

$C M O D^{\prime}$ is simple for assignments and for procedure calls is analogous to $P M O D^{\prime}$.

$$
\begin{aligned}
& C M O D^{\prime}(n, R A)= \begin{cases}\operatorname{CondLMOD}^{\prime}(n, R A) & \text { if } n \text { is an assignment } \\
\mathcal{S}_{(n, R A)} & \begin{array}{l}
\text { if } n \text { is a call of } \mathrm{Q} \\
\text { otherwise }
\end{array}\end{cases} \\
& \text { where } \mathcal{S}_{(n, R A)}=\bigcup_{\left(R A^{\prime}, b^{\prime}\right) \in \text { contexts_of }(n, R A)}\left\{\begin{array}{ll}
(o b j, b) & \begin{array}{l}
\left(o b j^{\prime}, b^{\prime \prime}\right) \in P M O D^{\prime}\left(Q, R A^{\prime}\right) \wedge \\
o b j \in b_{n}\left(\left\{o b j^{\prime}\right\}\right) \wedge b=b^{\prime} \sqcap_{1} b^{\prime \prime}
\end{array}
\end{array}\right\}
\end{aligned}
$$

Finally, $M O D^{\prime}$ is $(n$ is in procedure $P$ ) 


$$
\begin{aligned}
& \operatorname{MOD}^{\prime}(n)=\bigcup_{\text {reaching alias } R A \text { for } P} \operatorname{CMOD}^{\prime}(n, R A) \\
& \operatorname{MOD}^{\prime}(P)=\bigcup_{\text {reaching alias } R A \text { for } P} \operatorname{PMOD}^{\prime}(P, R A)
\end{aligned}
$$

\section{RAW DATA}

Table III shows the analysis times for the $\mathrm{MOD}_{C}$ calculations broken into two passes, and for a simple compilation using Gnu's gcc compiler version 2.7.2 with no optimizations enabled. The numbers are as reported by the UNIX time utility, averaged over 5 executions on a Sun Sparcstation 20 with $348 \mathrm{MB}$ of RAM and 527MB swap space. The $\operatorname{MOD}_{C}(\mathrm{FS})$ and $\mathrm{MOD}_{C}(\mathrm{FI})$ times do not include the alias analysis times, but are simply the time taken to calculate the $\mathrm{MOD}_{C}$ solution given the alias solution. The total analysis time is the sum of the two columns (columns 4 and 6 for $\operatorname{MOD}_{C}$ (FSAlias) and columns 5 and 7 for $\operatorname{MOD}_{C}($ FIAlias $)$ ).

Tables IV to XI contain summary statistics for the MOD solution. These statistics are subdivided with respect to the type of fixed locations being modified. There are five types:

- glo: MOD information for global variables.

- dyn: MOD information for dynamic storage locations (heap storage creation sites).

- loc: MOD information for local variables of the enclosing procedure (including formal parameters).

- nv: (non-visible) MOD information for local variables of other procedures or of an earlier recursive instantiation of the enclosing procedure. Section 2.2 discusses non-visibles in more detail.

- tot: MOD information for all fixed locations.

There are three different summary statistics. Average \# Location Modified (Maximum \# Location Modified) is the average (maximum) number of fixed locations modified by statements (possibly procedures) of the type indicated by the tables. Average Percent of Worst Case is more complicated. The number of fixed locations potentially modified by an assignment is the sum of

- the number of globals in the program,

- the number of dynamic allocation sites,

- the number of locals in the enclosing procedure, and

- the number of locals of other procedures (including locals of earlier recursive instantiations of this procedure) accessible through globals and formals at the entry of the enclosing procedure.

Percent of Worst Case is simply the number of fixed locations modified divided by the number of potentially modified locations. The Average Percent of Worst Case is the average of Percent of Worst Case over all assignments, calls, or procedures depending on the statement kind. For some 
Table III. Timing Data

\begin{tabular}{|c|c|c|c|c|c|c|}
\hline Program & $\begin{array}{l}\text { ICFG } \\
\text { Nodes }\end{array}$ & $\begin{array}{l}\text { Compile } \\
\text { Time(s) }\end{array}$ & $\begin{array}{c}\text { FSAlias } \\
\text { Time(s) }\end{array}$ & $\begin{array}{l}\text { FIAlias } \\
\text { Time(s) }\end{array}$ & $\begin{array}{c}\mathrm{MOD}_{C}(\mathrm{FS}) \\
\operatorname{Time}(\mathrm{s}) \\
\end{array}$ & $\begin{array}{c}\mathrm{MOD}_{C}(\mathrm{FI}) \\
\text { Time }(\mathrm{s}) \\
\end{array}$ \\
\hline allroots & 422 & 2.42 & 2.34 & 0.08 & 0.02 & 0.02 \\
\hline fixoutput & 617 & 1.98 & 1.18 & 0.07 & 0.03 & 0.03 \\
\hline diffh & 646 & 1.62 & 1.70 & 0.11 & 0.04 & 0.03 \\
\hline travel & 698 & 2.04 & 3.34 & 0.12 & 0.04 & 0.05 \\
\hline $\mathrm{ul}$ & 1027 & 1.72 & 3.15 & 0.13 & 0.08 & 0.05 \\
\hline plot2fig & 1077 & 15.68 & 3.80 & 0.18 & 0.06 & 0.07 \\
\hline lex315 & 1297 & 2.10 & 3.64 & 0.14 & 0.08 & 0.07 \\
\hline compress & 1319 & 2.10 & 3.16 & 0.18 & 0.08 & 0.08 \\
\hline clinpack & 1429 & 2.42 & 7.34 & 0.23 & 0.12 & 0.09 \\
\hline loader & 1563 & 5.42 & 6.18 & 0.29 & 0.12 & 0.15 \\
\hline mway & 1576 & 3.12 & 4.59 & 0.21 & 0.10 & 0.11 \\
\hline ansitape & 1747 & 3.24 & 5.13 & 0.39 & 0.11 & 0.12 \\
\hline stanford & 1771 & 2.46 & 2.53 & 0.22 & 0.10 & 0.09 \\
\hline pokerd & 1895 & 7.90 & 26.52 & 0.29 & 0.14 & 0.11 \\
\hline zipship & 1955 & 2.66 & 4.33 & 0.27 & 0.20 & 0.13 \\
\hline dixie & 2341 & 10.64 & 8.09 & 0.30 & 0.18 & 0.23 \\
\hline zipnote & 2407 & 9.50 & 19.40 & 0.53 & 0.64 & 0.16 \\
\hline learn & 2626 & 8.24 & 6.27 & 0.37 & 0.18 & 0.21 \\
\hline xmodem & 2672 & 6.00 & 4.81 & 0.31 & 0.21 & 0.19 \\
\hline compiler & 3008 & 7.84 & 3.06 & 0.19 & 0.36 & 0.35 \\
\hline zipcloak & 3033 & 7.50 & 20.42 & 0.64 & 0.67 & 0.19 \\
\hline $\operatorname{sim}$ & 3034 & 3.00 & 8.24 & 0.35 & 0.31 & 0.20 \\
\hline cdecl & 3196 & 4.60 & 21.08 & 0.33 & 0.34 & 0.25 \\
\hline diff & 3300 & 4.36 & 11.17 & 0.50 & 0.32 & 0.21 \\
\hline unzip & 3416 & 9.88 & 10.57 & 0.40 & 0.27 & 0.29 \\
\hline assembler & 3601 & 11.54 & 26.06 & 0.55 & 0.57 & 0.56 \\
\hline gnugo & 3651 & 14.24 & 3.25 & 0.32 & 0.19 & 0.19 \\
\hline livc & 4101 & 4.50 & 7.98 & 0.57 & 0.26 & 0.37 \\
\hline lharc & 4250 & 4.62 & 12.72 & 0.79 & 0.34 & 0.36 \\
\hline patch & 4608 & 6.94 & 18.80 & 0.51 & 0.35 & 0.40 \\
\hline simulator & 5574 & 14.70 & 11.31 & 0.60 & 0.34 & 0.53 \\
\hline arc & 5856 & 19.02 & 15.00 & 0.91 & 0.44 & 0.53 \\
\hline triangle & 6119 & 8.42 & 8.88 & 0.64 & 0.72 & 0.31 \\
\hline tbl & 6162 & 14.00 & 27.16 & 0.54 & 0.67 & 0.43 \\
\hline football & 7313 & 10.22 & 9.86 & 0.83 & 0.37 & 0.46 \\
\hline flex & 7376 & 11.46 & 116.82 & 0.74 & 0.82 & 0.67 \\
\hline zip & 9288 & 18.12 & & 1.80 & & 1.01 \\
\hline $072 . s c$ & 13690 & 16.52 & & 2.05 & & 2.08 \\
\hline spim & 16740 & 25.38 & & 2.23 & & 3.78 \\
\hline larn & 21184 & 37.78 & & 2.36 & & 8.03 \\
\hline tsl & 27302 & 21.72 & & 10.86 & & 14.69 \\
\hline 008.espresso & 30510 & 40.72 & & 6.17 & & 6.09 \\
\hline moria & 38572 & 45.84 & 77.89 & 7.02 & 3.02 & 52.07 \\
\hline TWMC & 51627 & 152.22 & & 11.06 & & 9.47 \\
\hline nethack & 58317 & 71.38 & & 124.32 & & 355.41 \\
\hline
\end{tabular}




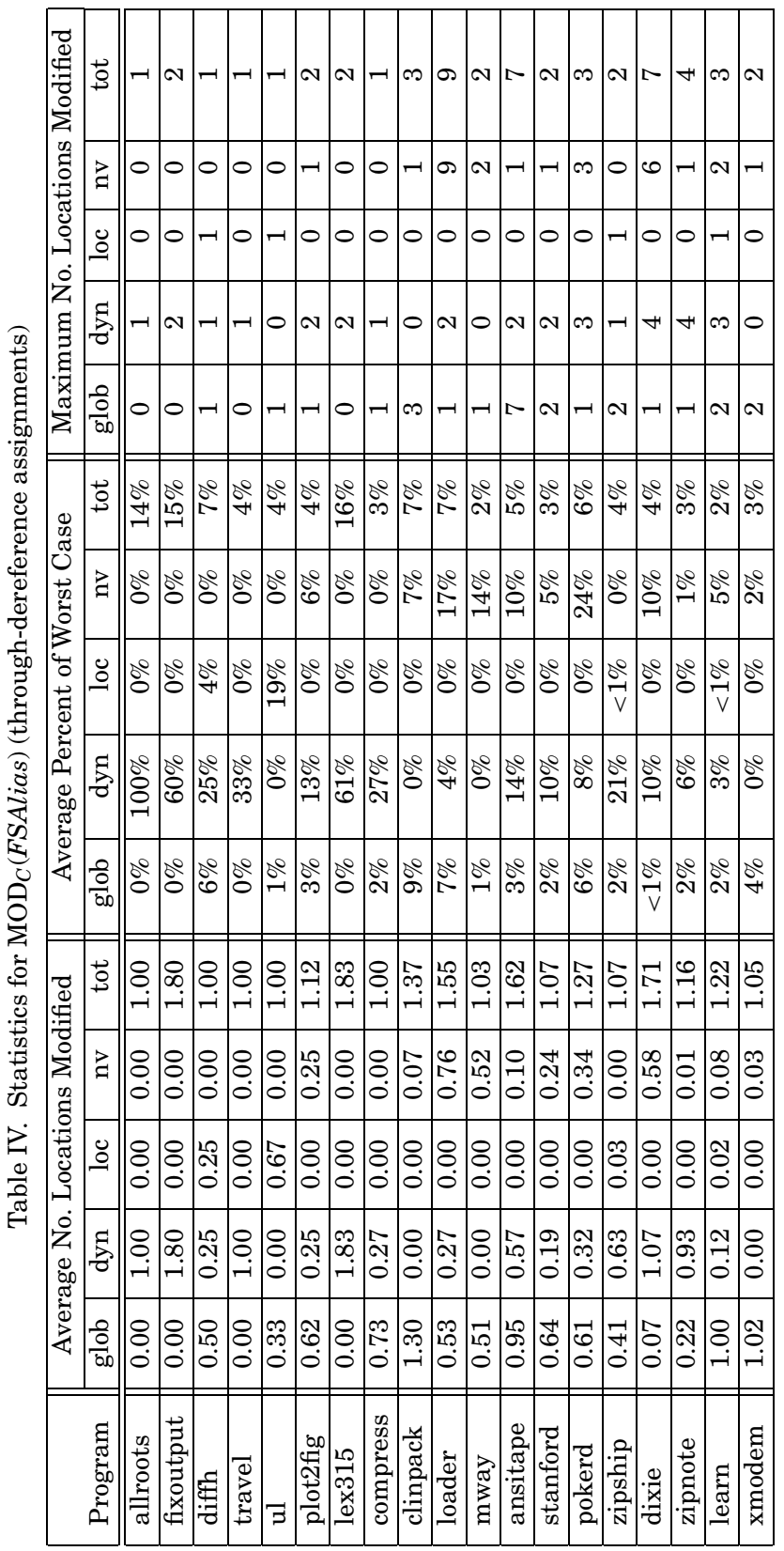




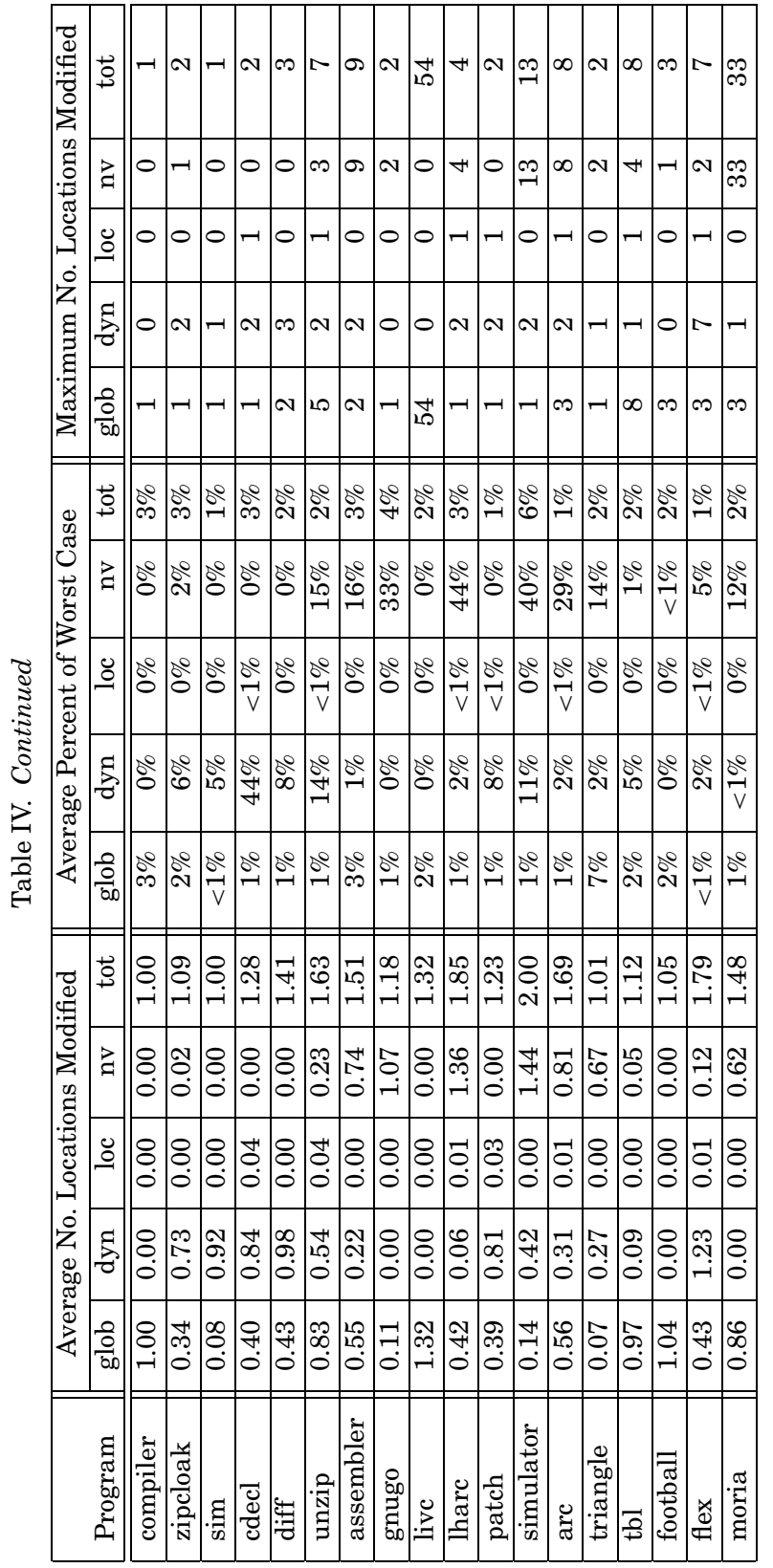




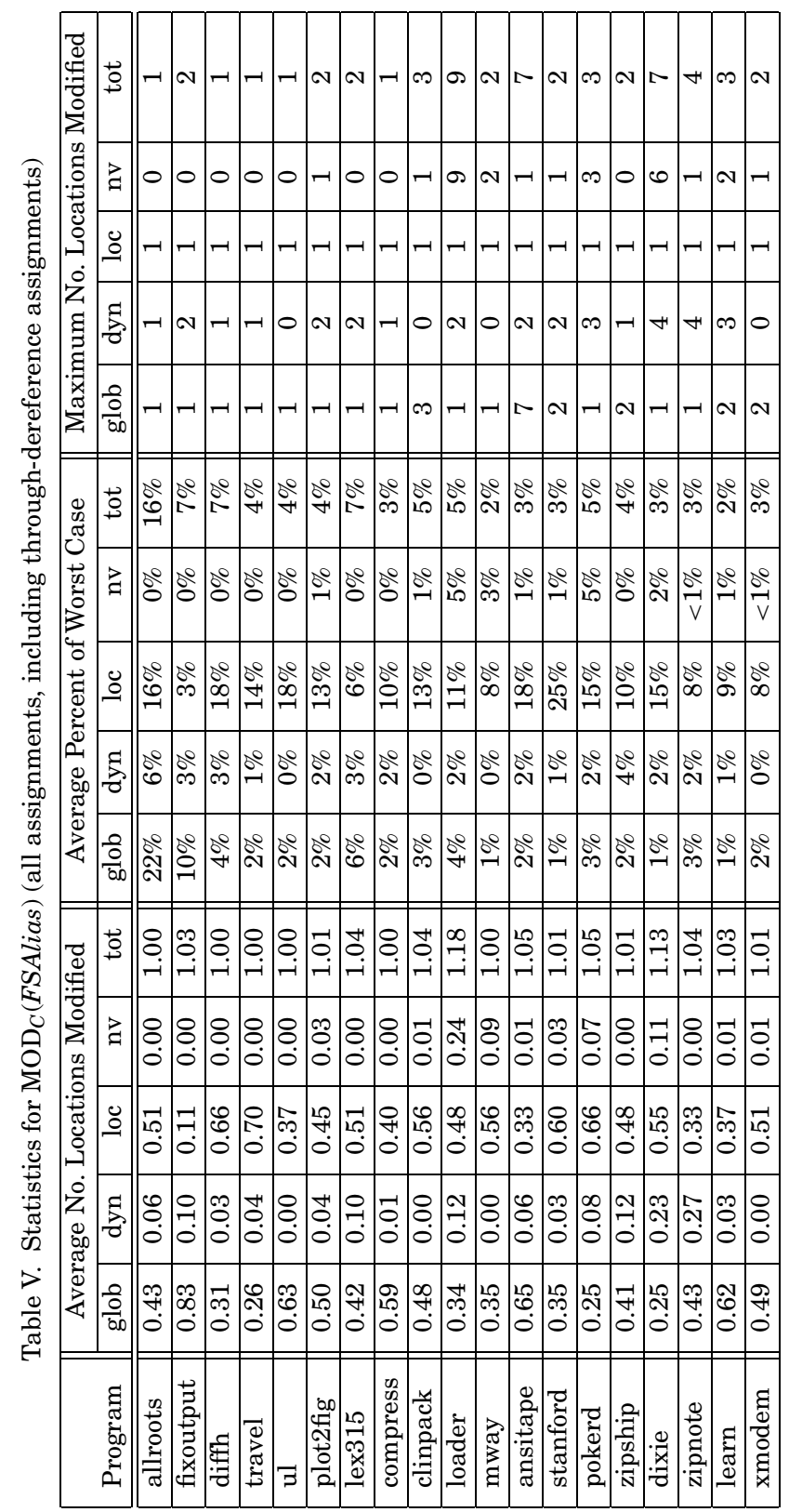




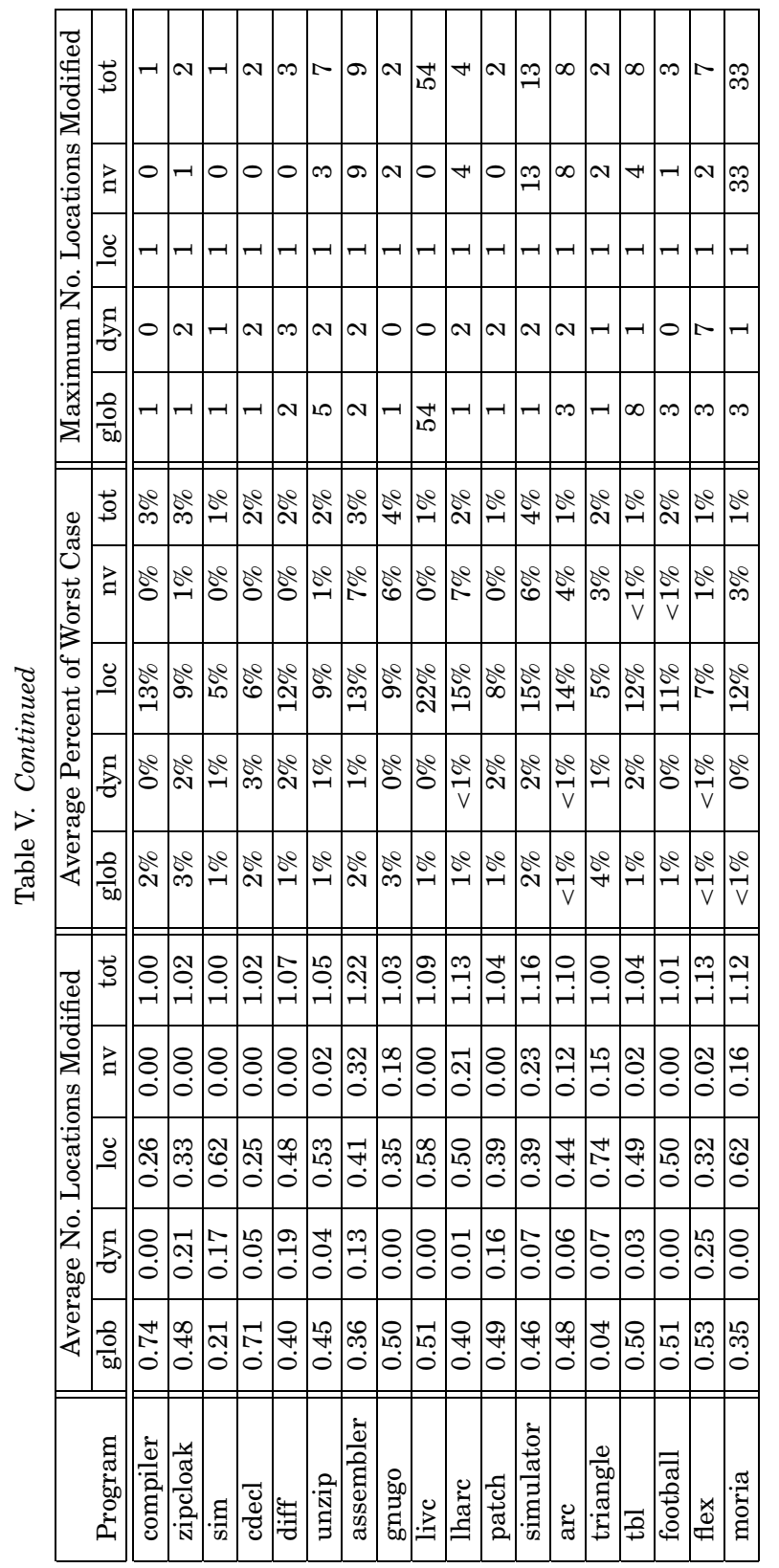




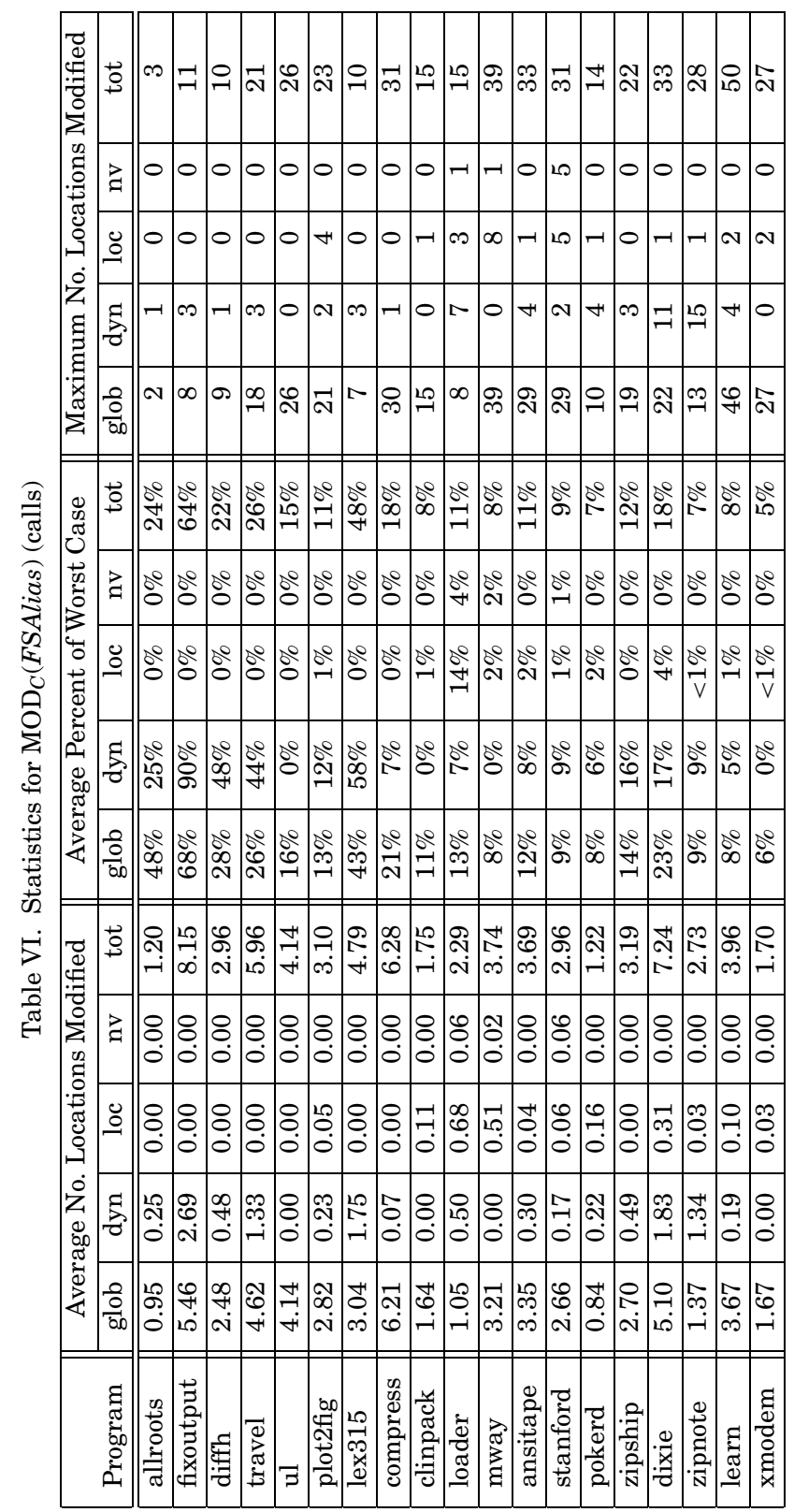




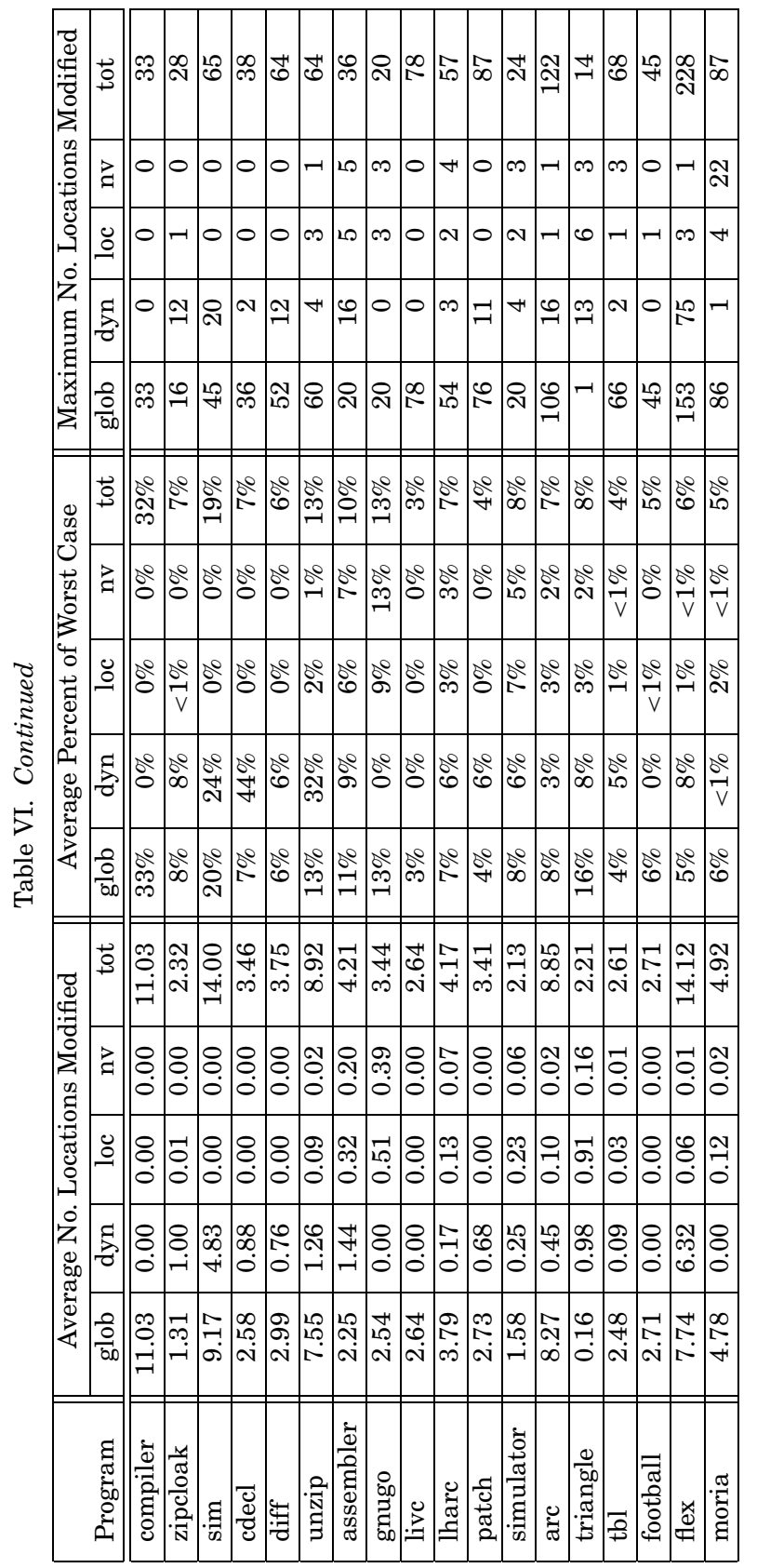




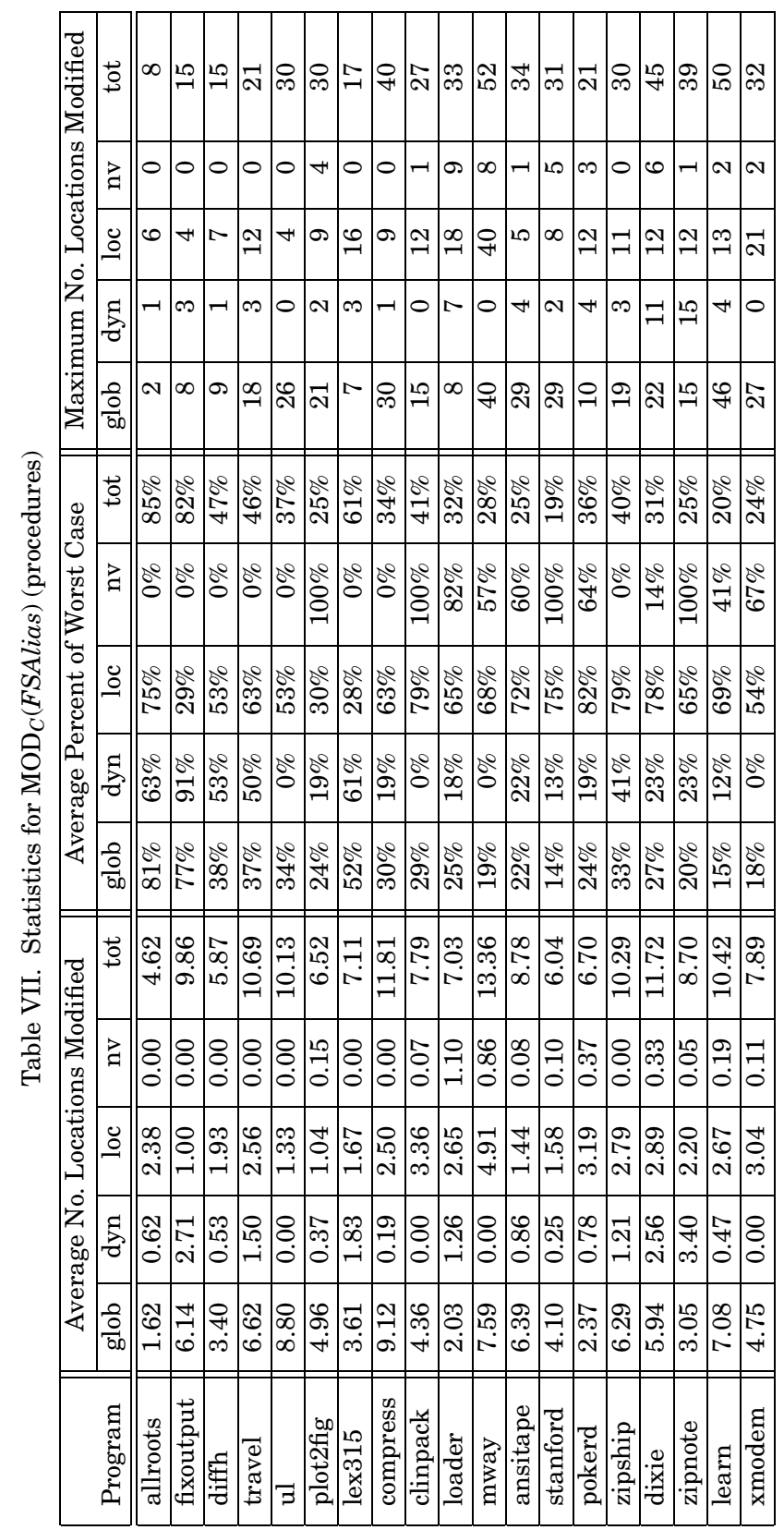




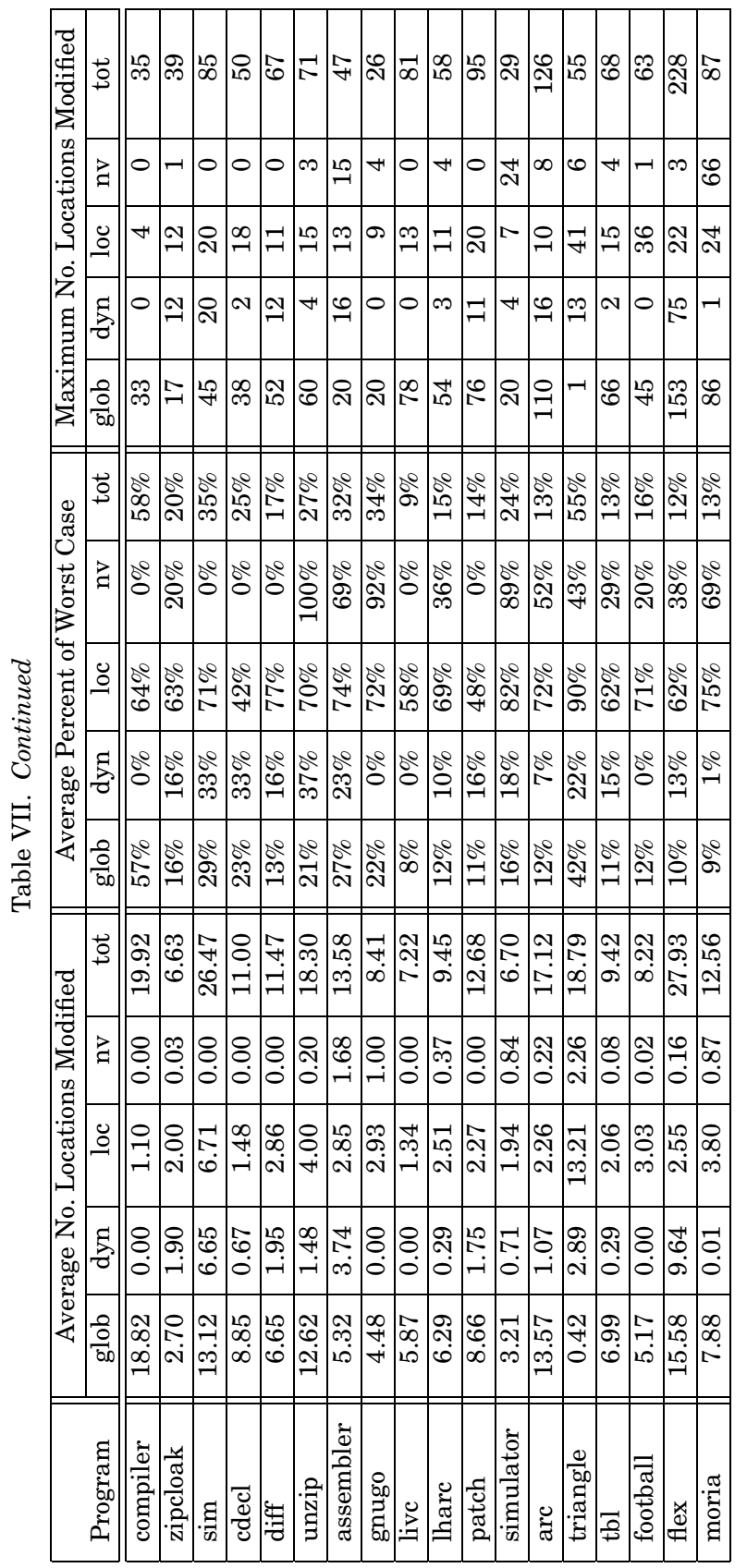




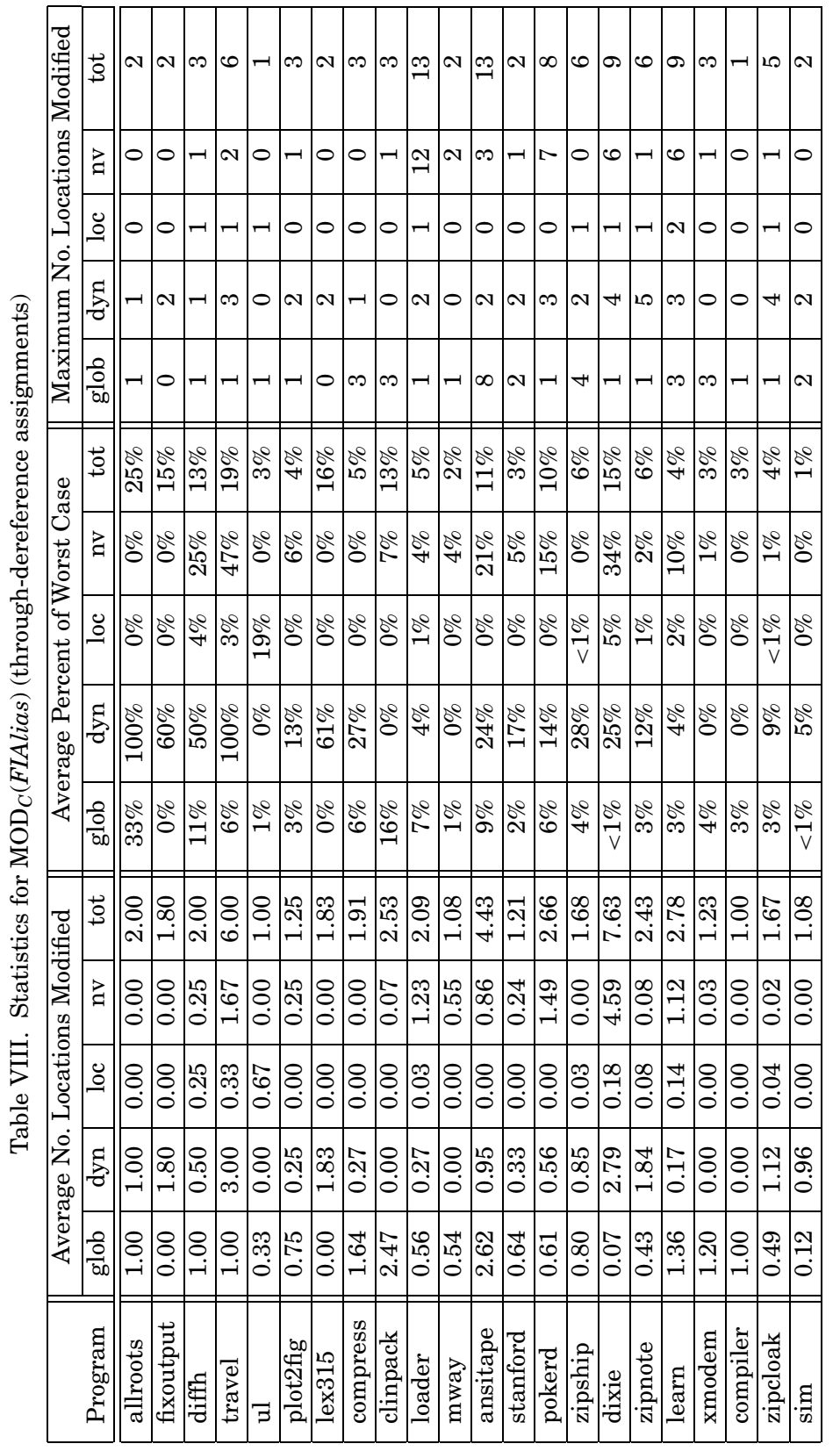




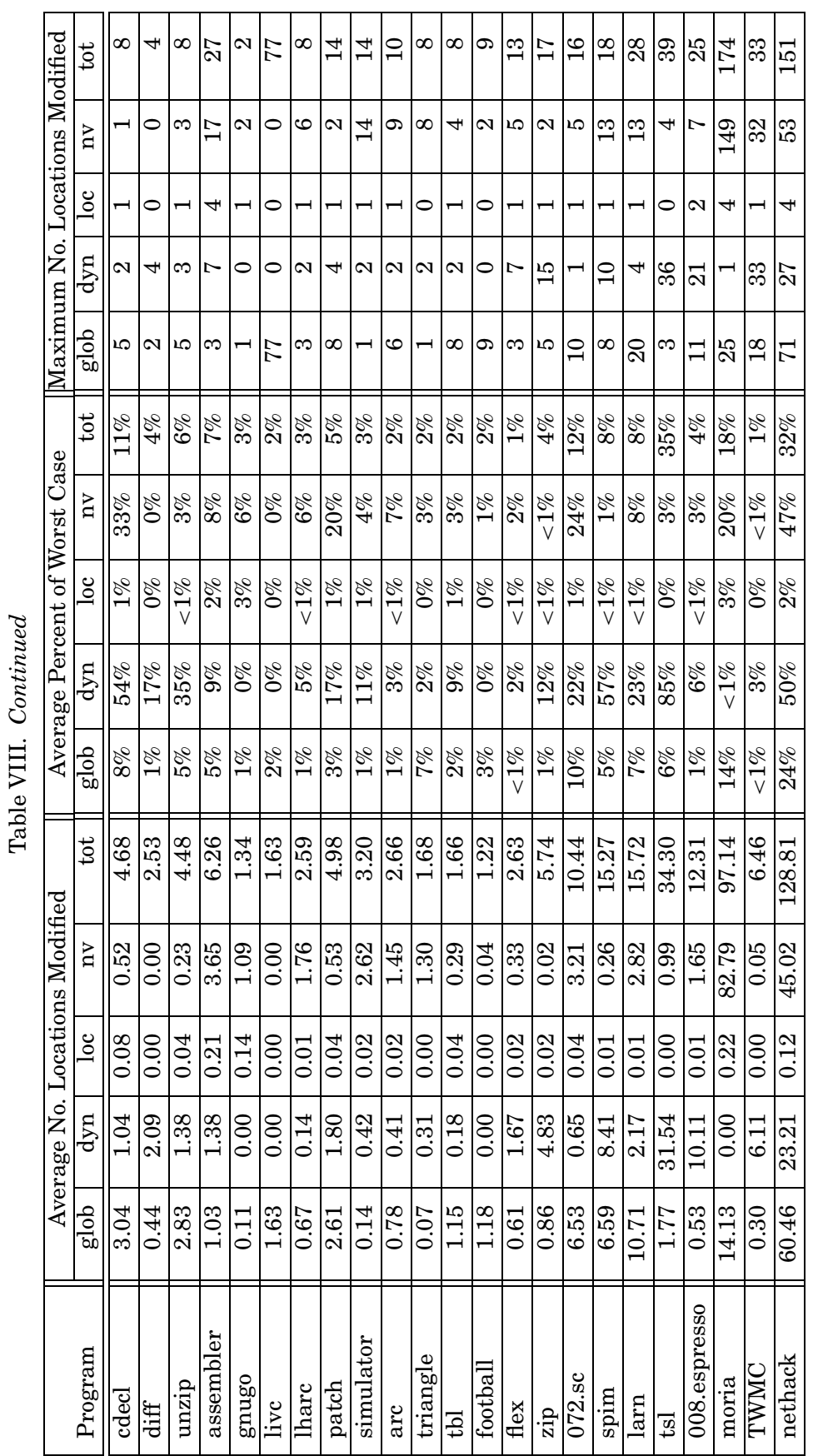

ACM Transactions on Programming Languages and Systems, Vol. 23, No. 1, March 2001. 


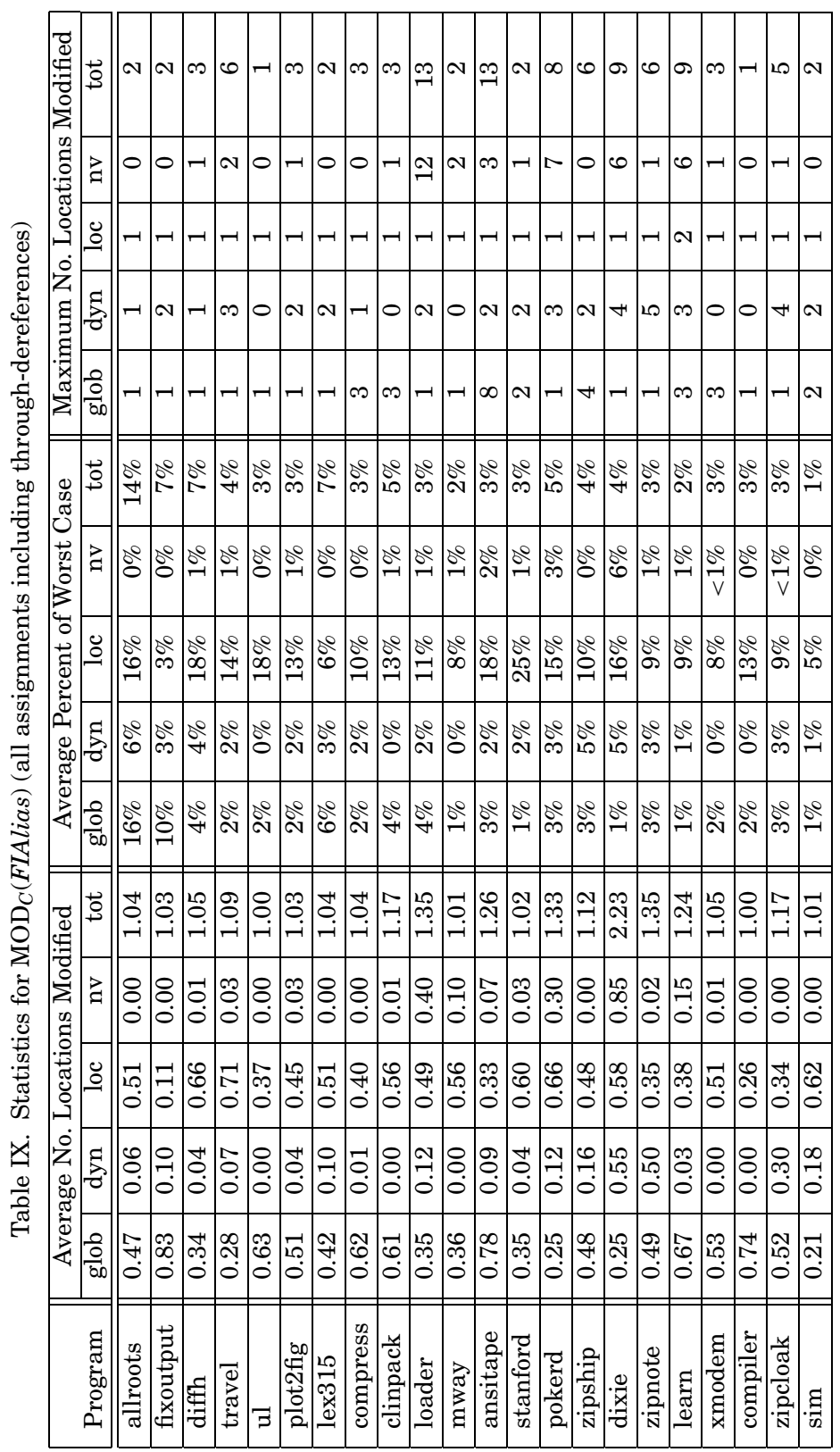




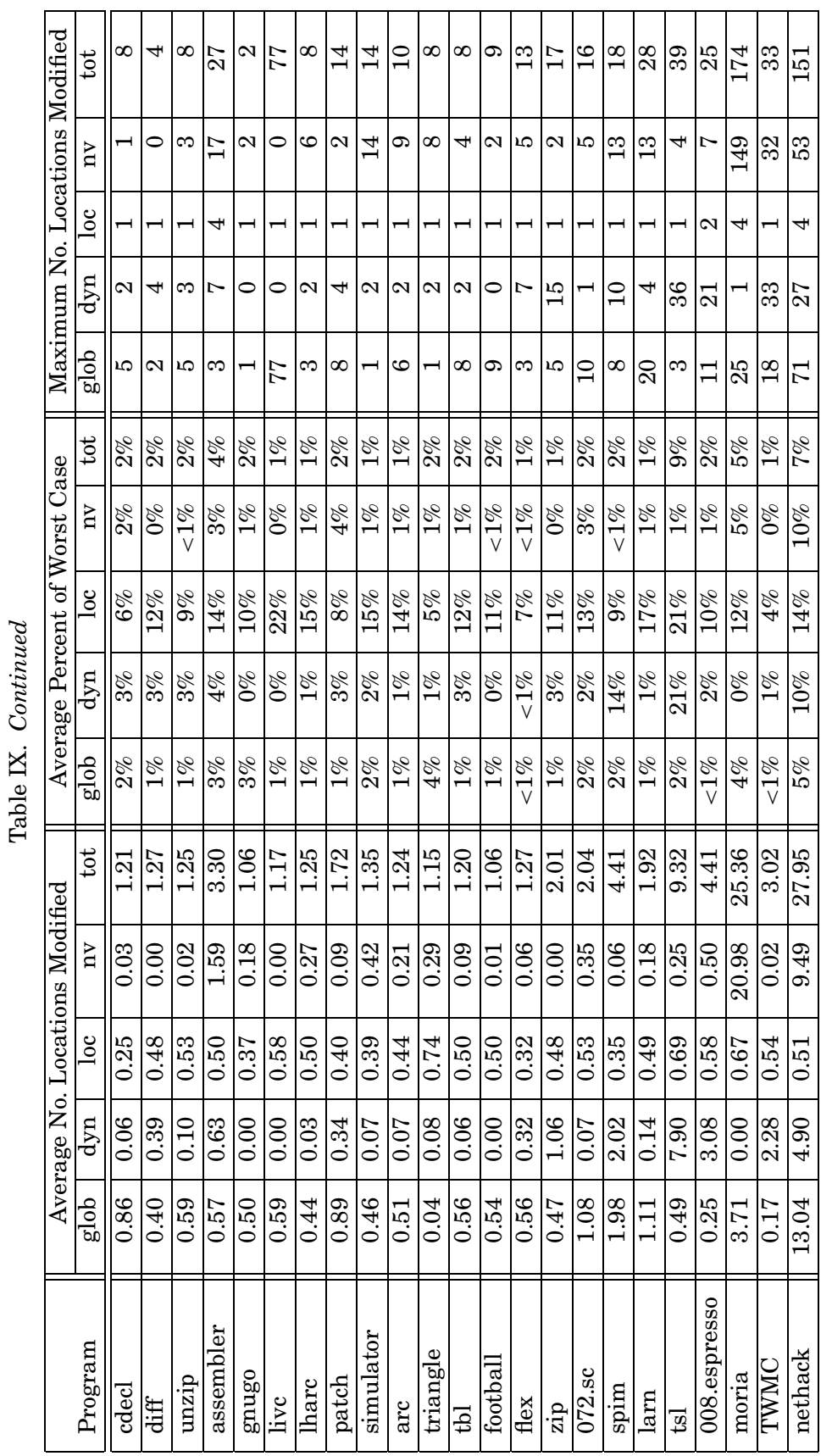




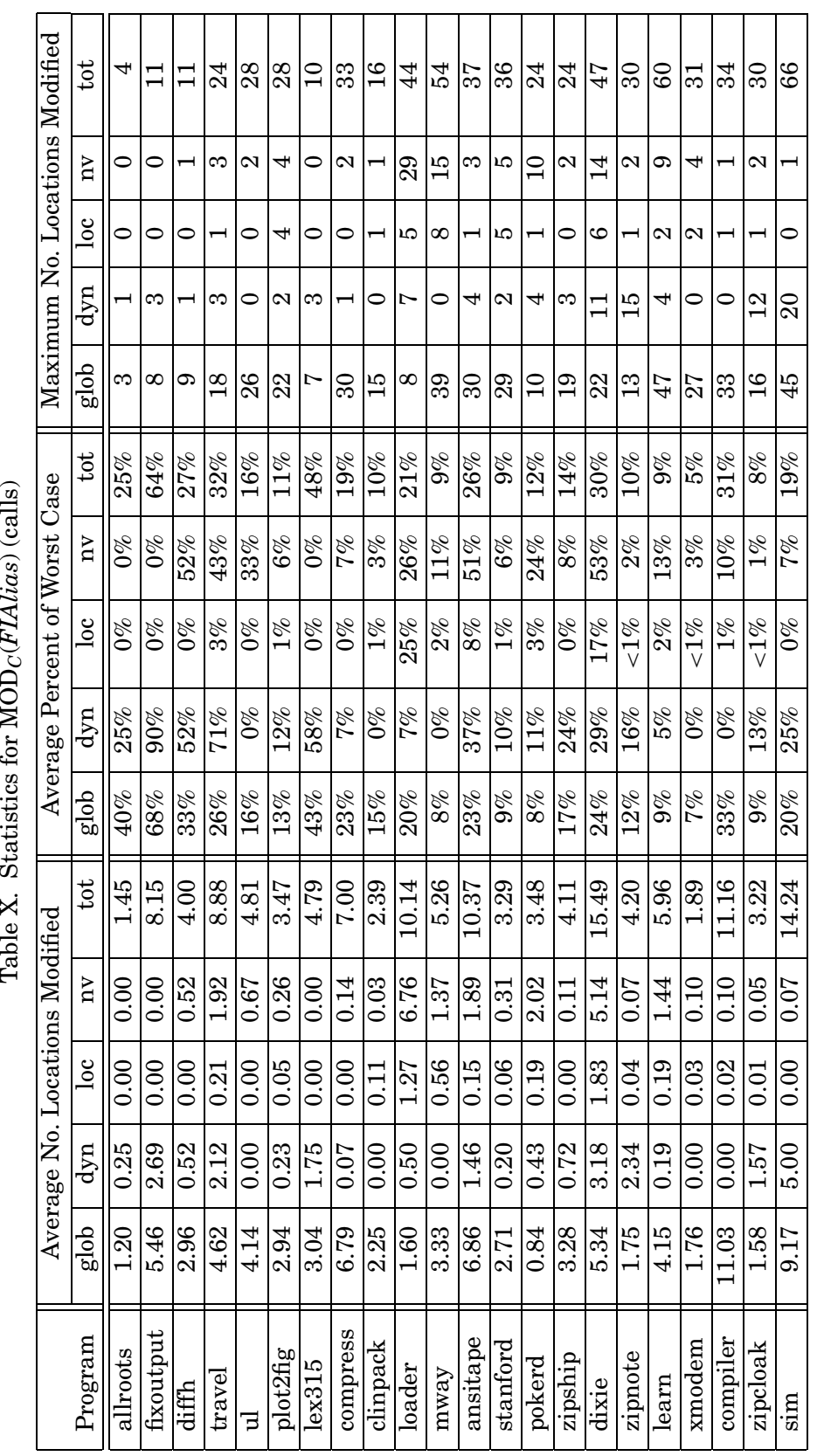




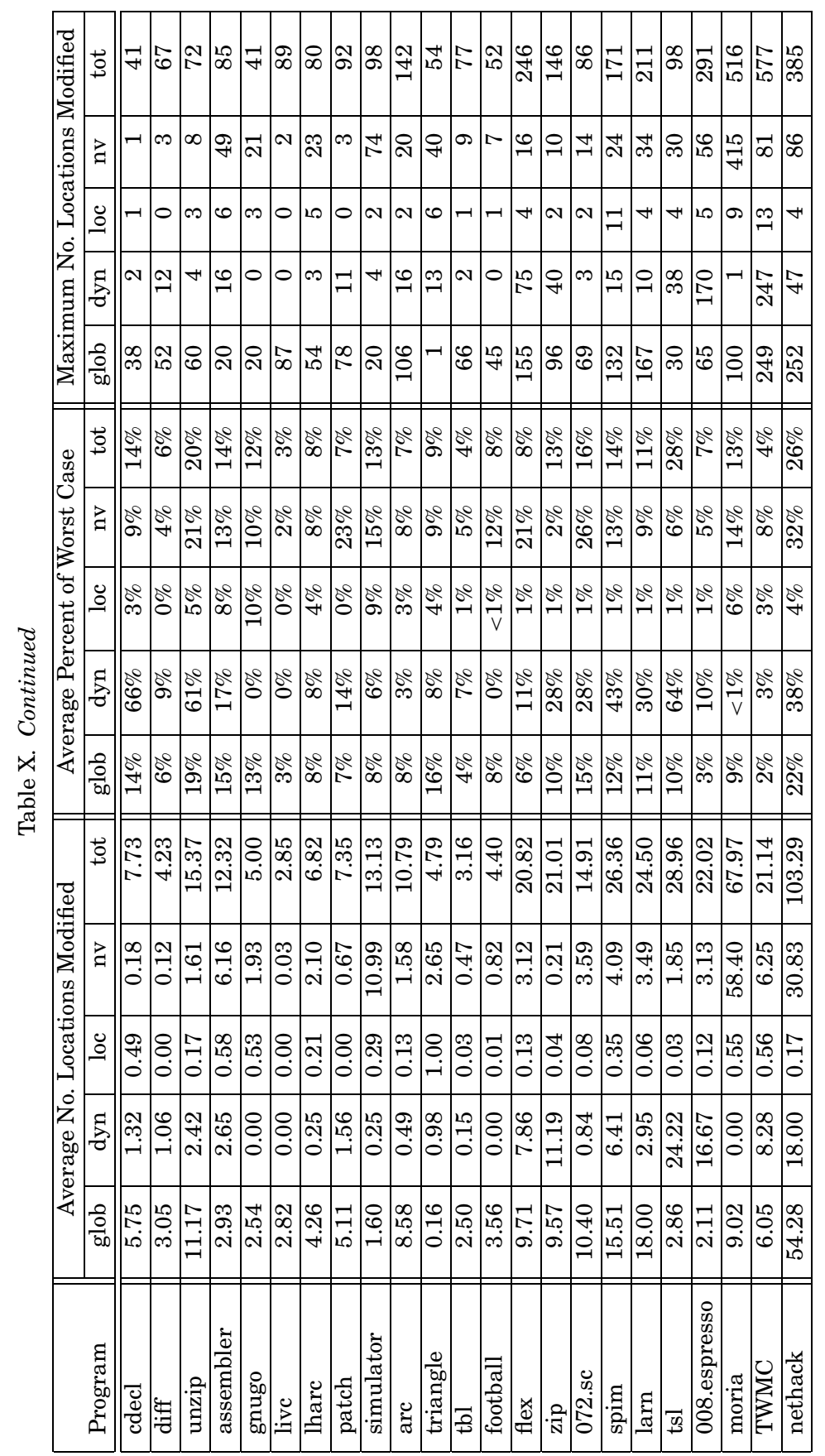




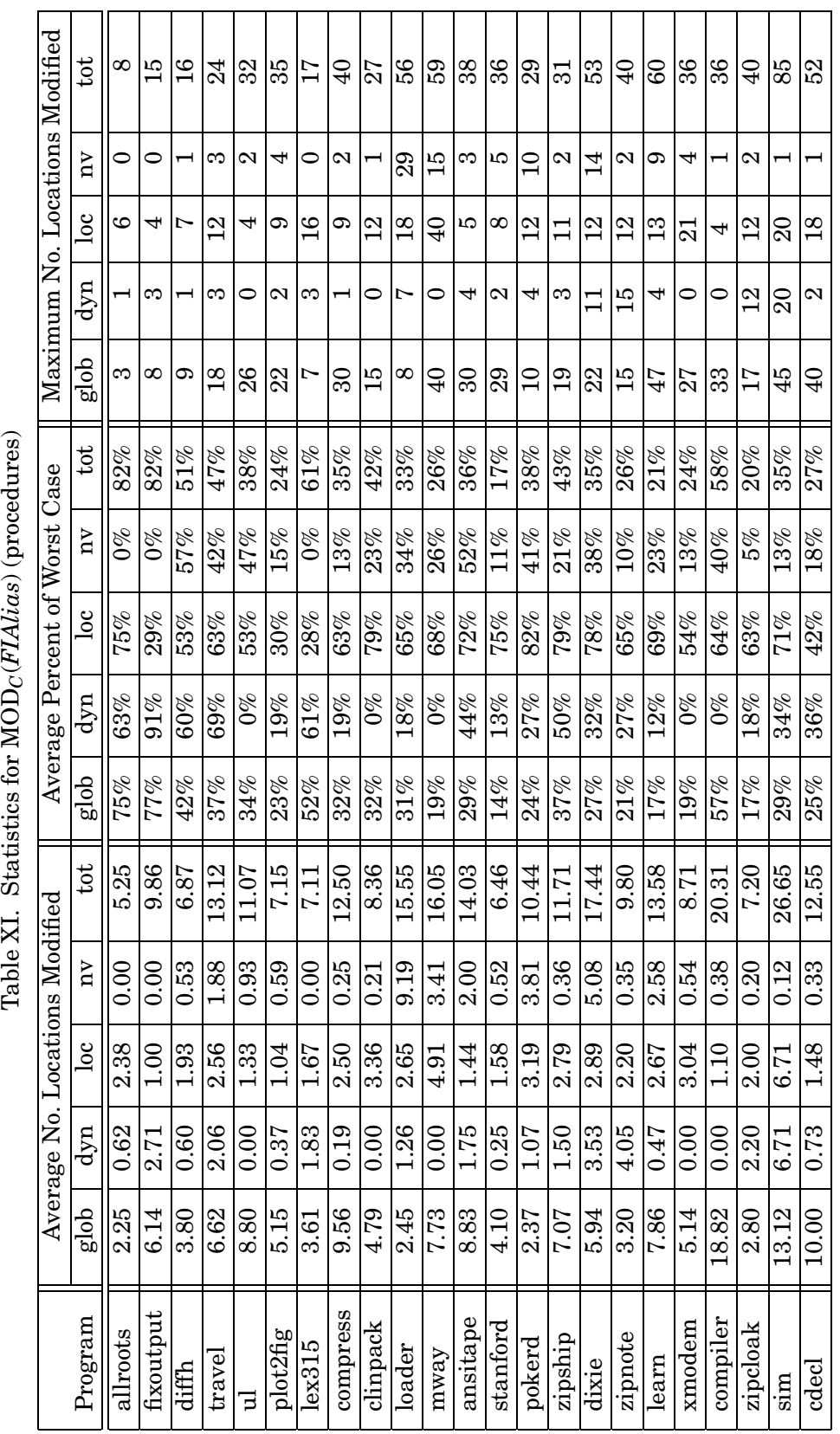




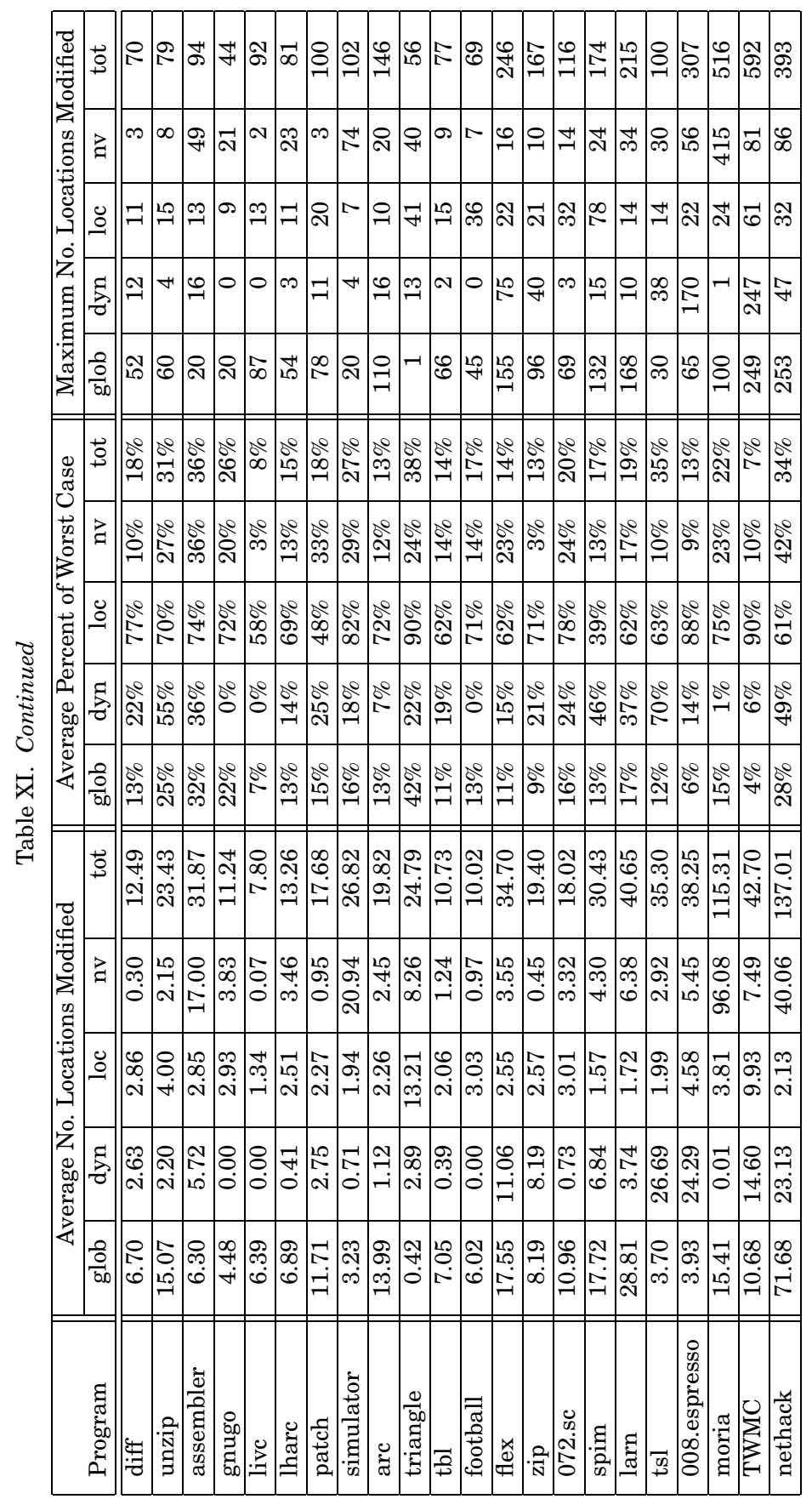


statements/procedures, the number of possible locals and the number of non-visibles are zero. In these cases, "0\%" is used as the percent/assign.

\section{ACKNOWLEDGMENTS}

The authors acknowledge the contributions of Tom Marlowe to the comparative examples in Section 2 and Jyh-shiarn Yur to the description of FSAlias in Section 2. The authors also thank Atanas Rountev and Ulrich Kremer for their helpful discussions.

\section{REFERENCES}

Aho, A. V., Sethi, R., and Ullman, J. D. 1986. Compilers: Principles, Techniques, and Tools. Addison-Wesley, Reading, MA.

Allen, F. E. 1974. Interprocedural data flow analysis. In Proceedings of 1974 IFIP Congress, Amsterdam, Holland, pp. 398-402. Institute of Electrical and Electronics Engineers, Inc., North Holland Publishing Company.

Andersen, L. O. 1994. Program analysis and specialization for the C programming language. Ph.D. Thesis, DIKU, University of Copenhagen. Also available as DIKU report 94/19.

Atkinson, D. ANd GRiswold, W. 1996. The design of whole-program analysis tools. In Proceedings of the 18th International Conference on Software Engineering, pp. 16-27.

AtKinson, D. AND GRISwold, W. 1998. Effective whole-program analysis in the presence of pointers. In Proceedings of the ACM SIGSOFT' 98 Symposium on the Foundations of Software Engineering, pp. 46-55.

BanerJee, U. 1988. Dependence Analysis for Supercomputing. Kluwer Academic Publishers, Norwell, MA.

BANNing, J. 1979. An efficient way to find the side effects of procedure calls and the aliases of variables. In Conference Record of the Sixth Annual ACM SIGACT/SIGPLAN Symposium on Principles of Programming Languages. pp. 29-41.

BARTH, J. M. 1978. A practical interprocedural data flow analysis algorithm. Communications of the ACM 21, 9, 724-736.

Bates, S. AND HoRwitz, S. 1993. Incremental program testing using dependence graphs. In Conference Record of the Twentieth Annual ACM SIGACT/SIGPLAN Symposium on Principles of Programming Languages, pp. 384-396.

Burke, M. 1990. An interval-based approach to exhaustive and incremental interprocedural data flow analysis. ACM Transactions on Programming Languages and Systems, 12, 3, 341-395.

BuRKE, M. AND RYDER, B. G. 1990. A critical analysis of incremental iterative data flow analysis algorithms. IEEE Transactions on Software Engineering, 16, 7.

Burke, M., CARINI, P., ChoI, J.-D., AND Hind, M. 1994. Flow-insensitive interprocedural alias analysis in the presence of pointers. In Proceedings of the Seventh International Workshop on Languages and Compilers for Parallel Computing, pp. 234-250. Springer-Verlag.

Burke, M., CARini, P., Choi, J.-D., AND Hind, M. 1997. Interprocedural pointer alias analysis. Research Report RC 21055, IBM T. J. Watson Research Center.

CARRoll, M. D. 1988. A new pointer-removing program transformation. Unpublished manuscript.

Carroll, M. D. And Ryder, B. G. 1988. Incremental data flow analysis via dominator and attribute updates. In Conference Record of the Fifteenth Annual ACM SIGACT/SIGPLAN Symposium on Principles of Programming Languages, pp. 274-284.

Chase, D. R., Wegman, M., AND Zadeck, F. K. 1990. Analysis of pointers and structures. In Proceedings of the SIGPLAN '90 Conference on Programming Language Design and Implementation, pp. 296-310. SIGPLAN Notices, Vol. 25, No. 6.

Chatterjee, R. 1999. Modular data-flow analysis of statically typed object-oriented programming languages. Ph.D. Thesis, Department of Computer Science, Rutgers University.

Chattersee, R. AND RYder, B. G. 1999. Data-flow-based testing of object-oriented libraries. Department of Computer Science Technical Report DCS-TR-382, Rutgers University. 
Chatterjee, R., Ryder, B. G., And Landi, W. A. 1999. Relevant context inference. In Conference Record of the Twenty-Sixth Annual ACM SIGACT/SIGPLAN Symposium on Principles of Programming Languages.

CHOI, J.-D., BuRKe, M., AND CARINI, P. 1993. Efficient flow-sensitive interprocedural computation of pointer-induced aliases and side effects. In Conference Record of the Twentieth Annual ACM SIGACT/SIGPLAN Symposium on Principles of Programming Languages, pp. 232-245.

Cooper, K. 1985. Analyzing aliases of reference formal parameters. In Conference Record of the Twelfth Annual ACM SIGACT/SIGPLAN Symposium on Principles of Programming Languages, pp. 281-290.

Cooper, B. G. 1989. Ambitious data flow analysis of procedural programs. Master's Thesis, University of Minnesota.

CoOper, K. AND KENNEDY, K. 1984. Efficient computation of flow insensitive interprocedural summary information. In Proceedings of the ACM SIGPLAN Symposium on Compiler Construction, pp. 247-258. SIGPLAN Notices, Vol. 19, No. 6.

Cooper, K. And Kennedy, K. 1987. Complexity of interprocedural side-effect analysis. Computer Science Department Technical Report TR87-61, Rice University.

Cooper, K. AND Kennedy, K. 1988. Interprocedural side-effect analysis in linear time. In Proceedings of the SIGPLAN '88 Conference on Programming Language Design and Implementation, pp. $57-66$.

Coutant, D. S. 1986. Retargetable high-level alias analysis. In Conference Record of the Thirteenth Annual ACM SIGACT/SIGPLAN Symposium on Principles of Programming Languages, pp. 110-118.

DAS, M. 2000. Unification-based pointer analysis with directional assignments. In Proceedings of the ACM SIGPLAN 'OO Conference on Programming Language Design and Implementation, pp. 35-46.

Deutsch, A. 1990. On determining lifetime and aliasing of dynamically allocated data in higherorder functional specifications. In Conference Record of the Seventeenth Annual ACM SIGACT/ SIGPLAN Symposium on Principles of Programming Languages, pp. 157-168.

Deutsch, A. 1992. A storeless model of aliasing and its abstractions using finite representations of right-regular equivalence relations. In Proceedings of the IEEE 1992 Conference on Computer Languages, pp. 2-13.

Deutsch, A. 1994. Interprocedural may alias for pointers: Beyond k-limiting. In Proceedings of the SIGPLAN '94 Conference on Programming Language Design and Implementation, pp. 230241.

Duesterwald, E., Gupta, R., And Soffa, M. L. 1995. Demand-driven computation of interprocedural data flow. In Conference Record of the Twenty-Second Annual ACM SIGACT/SIGPLAN Symposium on Principles of Programming Languages.

Duesterwald, E., Gupta, R., AND Soffa, M. L. 1996. A demand-driven analyzer for data flow testing at the integration level. In Proceedings of the International Conference on Software Engineering (ICSE'96).

EмAмI, M. 1993. A practical interprocedural alias analysis for an optimizing/parallelizing C compiler. Master's Thesis, McGill University, Montreal, Canada.

Emami, M., Ghiya, R., ANd Hendren, L. J. 1994. Context-sensitive interprocedural points-to analysis in the presence of function pointers. In Proceedings of the SIGPLAN '94 Conference on Programming Language Design and Implementation, pp. 242-257. Published as SIGPLAN Notices, 29 (6).

FAHNDRICH, M., REHOF, J., AND DAS, M. 2000. Scalable context-sensitive flow analysis using instantiation constraints. Proceedings of the ACM SIGPLAN'00 Conference on Programming Language Design and Implementation, pp. 253-263.

Frankel, P. And Iakounenko, O. 1998. Further empirical studies of test effectiveness. In ACM SIGSOFT '98 Sixth International Symposium on the Foundations of Software Engineering, pp. 153-162.

Frankel, P. AND Weiss, S. 1993. An experimental comparison of the effectiveness of branch testing with data-flow testing. IEEE Transactions on Software Engineering 19, 8, 774-787.

Foster, J., Fahndrich, M., And Aiken, A. 2000. Polymorphic versus monomorphic flow-insensitive points-to analysis for C. In Proceedings of International Symposium on Static Analysis (SAS '00). 
Gallagher, K. And Lyle, J. 1991. Using program slices in software maintenance. IEEE Transactions on Software Engineering 17, 8, 751-761.

Ghiya, R. And Hendren, L. 1996a. Connection analysis: A practical interprocedural heap analysis for C. International Journal of Parallel Programming.

Ghiya, R. And Hendren, L. 1996b. Is it a tree, a dag or a cyclic graph? A shape analysis for heap-directed pointers in C. In Conference Record of the Twenty-Third Annual ACM SIGACT/ SIGPLAN Symposium on Principles of Programming Languages, pp. 1-15.

Ghiya, R. And Hendren, L. 1998. Putting pointer analysis to work. In Conference Record of the Twenty-Fifth Annual ACM SIGACT/SIGPLAN Symposium on Principles of Programming Languages, pp. 121-133.

GuARnA, C. A. 1988. A technique for analyzing pointer and structure references in parallel restructuring compilers. In Proceedings of the International Conference on Parallel Processing, pp. 212-220.

Gupta, R. ANd Soffa, M. L. 1996. Hybrid slicing: An approach for refining static slices using dynamic information. In Proceedings of Third ACM SIGSOFT Symposium on Foundations of Software Engineering, pp. 29-40.

Harrison, W. L., III and Ammarguellat, Z. 1990. Parcel and Miprac: Parallelizers for symbolic and numeric programs. In Proceedings of International Workshop on Compilers for Parallel Computers, pp. 329-346. Ecole des Mines de Paris-CAI, UPMC-Laboratoire MASI, Paris, France.

Harrold, M. J. ANd Ci, N. 1998. Reuse-driven interprocedural slicing. In Proceedings of the Twentieth International Conference on Software Engineering, pp. 74-83.

Harrold, M. J. and Soffa, M. L. 1991. Selecting and using data for integration testing. IEEE Software 8, 2, 58-65.

HaRrold, M. J. AND SofFa, M. L. 1994. Efficient computation of interprocedural definition-use chains. ACM Transactions on Programming Languages and Systems 16, 2, 175-204.

HASTI, R. AND HoRwitz, S. 1998. Using static single assignment form to improve flow-insensitive pointer analysis. In Proceedings of the ACM SIGPLAN'98 Conference on Programming Language Design and Implementation, pp. 97-105.

Hendren, L. ANd Nicolau, A. 1990. Parallelizing programs with recursive data structures. IEEE Transaction on Parallel and Distributed Systems.

Hendren, L., Hummel, J., AND Nicolau, A. 1992. Abstractions for recursive pointer data structures: Improving analysis and transformations of imperative languages. In Proceedings of the SIGPLAN '92 Conference on Programming Language Design and Implementation, pp. 249-260. SIGPLAN Notices, Vol. 27, No. 6.

Hind, M. AND Pioli, A. 1998. Assessing the effects of flow sensitivity on pointer alias analysis. In Proceedings of International Static Analysis Symposiusm (SAS'98), pp. 57-81. SpringerVerlag.

Hind, M. ANd Pioli, A. 2000. Evaluating the effectiveness of pointer alias analyses. In Proceedings of ACM SIGSOFT International Symposium on Software Testing and Analysis. Also available as IBM Research Center Technical Report RC21510.

Hind, M., BuRke, N., CARInI, P., AND ChoI, J.-D. 1999. Interprocedural pointer alias analysis. ACM Transactions on Programming Languages and Systems 21, 4, 848-894.

Horwitz, S., Pfeiffer, P., ANd Reps, T. 1989. Dependence analysis for pointer variables. In Proceedings of the ACM SIGPLAN Symposium on Compiler Construction, pp. 28-40.

HoRwitz, S., REPS, T., AND BinkLEy, D. 1990. Interprocedural slicing using dependence graphs. ACM Transactions on Programming Languages and Systems 12, 1.

HoRwitz, S., Reps, T., AND SAgiv, M. 1995. Demand interprocedural dataflow analysis. In Proceedings of the Third ACM SIGSOFT Symposium on the Foundations of Software Engineering, pp. 104-115.

Hutchins, M., Foster, H., Goradia, T., and Ostrand, T. 1994. Experiments on the effectiveness of dataflow- and controlflow-based test adequacy criteria. In Proceedings of the Sixteenth International Conference on Software Engineering, pp. 191-200.

Jones, N. D. ANd Muchnick, S. 1982a. Flow analysis and optimization of LISP-like structures. In Program Flow Analysis: Theory and Applications. S. Muchnick and N. Jones, Ed. Prentice Hall, Englewood, Cliff., NJ, pp. 102-131. 
Jones, N. D. And Muchnick, S. S. 1982b. A flexible approach to interprocedural data flow analysis and programs with recursive data structures. In Conference Record of the Ninth Annual ACM SIGACT/SIGPLAN Symposium on Principles of Programming Languages, pp. 66-74.

KAM, J. B. AND UlLman, J. D. 1977 . Monotone data flow analysis frameworks. Acta Informatica 7, pp. 305-317.

KILDALL, G. 1973. A unified approach to global program optimization. In Conference Record of the ACM SIGACT/SIGPLAN Symposium on Principles of Programming Languages, pp. 194-206.

LANDI, W. 1992a. Interprocedural aliasing in the presence of pointers. Ph.D. Thesis, Rutgers University. LCSR-TR-174.

LANDI, W. 1992b. Undecidability of static analysis. ACM Letters on Programming Languages and Systems 1, (4):323-337.

LANDI, W. AND RYder, B. G. 1991. Pointer-induced aliasing: A problem classification. In Conference Record of the Eighteenth Annual ACM SIGACT/SIGPLAN Symposium on Principles of Programming Languages, pp. 93-103.

LANDI, W. AND RYDER, B. G. 1992. A safe approximation algorithm for interprocedural pointer aliasing. In Proceedings of the SIGPLAN '92 Conference on Programming Language Design and Implementation, pp. 235-248.

LANDI, W. AND RYDER, B. G. AND ZHANG, S. 1993. Interprocedural modification side effect analysis with pointer aliasing. In Proceedings of the SIGPLAN '93 Conference on Programming Language Design and Implementation, pp. 56-67.

Larsen, L. ANd Harrold, M. J. 1996. Slicing object-oriented software. In Proceedings of the Eighteenth International Conference on Software Engineering, pp. 495-505.

Larus, J. R. ANd Hilfinger, P. N. 1988. Detecting conflicts between structure accesses. In Proceedings of the SIGPLAN '88 Conference on Programming Language Design and Implementation, pp. 1-34. SIGPLAN NOTICES, Vol. 23, No. 7.

Liang, D. AND Harrold, M. J. 1999. Efficient points-to analysis for whole-program analysis. In Proceedings of the Seventh Annual ACM SIGSOFT Symposium on the Foundations of Software Engineering, LNCS, Vol. 1687, pp. 199-215.

Marlowe, T. J. AND Ryder, B. G. 1990a. An efficient hybrid algorithm for incremental data flow analysis. In Conference Record of the Seventeenth Annual ACM SIGACT/SIGPLAN Symposium on Principles of Programming Languages, pp. 184-196.

Marlowe, T. J. ANd Ryder, B. G. 1990b. Properties of data flow frameworks: A unified model. Acta Informatica $28,121-163$.

Marlowe, T. J. AND RYder, B. G. 1991. Hybrid incremental alias algorithms. In Proceedings of the Twenty-Fourth Hawaii International Conference on System Sciences, Vol. II, Software.

Marlowe, T. J., Landi, W. A., Ryder, B. G., Choi, J., Burke, M., and Carini, P. 1993. Pointer-induced aliasing: A clarification. ACM SIGPLAN Notices 28, 9, 67-70.

Neirynck, A., Panangaden, P., and Demers, A. 1987. Computation of aliases and support sets. In Conference Record of the Fourteenth Annual ACM SIGACT/SIGPLAN Symposium on Principles of Programming Languages, 274-283.

Ostrand, T. J. 1990. Data-flow testing with pointers and function calls. In Proceedings of the Pacific Northwest Software Quality Conference.

Ottenstein, K. J. and Ottenstein, L. M. 1984. The program dependence graph in a software development environment. In Proceedings of the ACM SIGSOFT/SIGPLAN Software Engineering Symposium on Practical Software Development Environments, pp. 177-184.

Pande, H. D., Landi, W., And Ryder, B. G. 1994. Interprocedural def-use associations for C systems with single level pointers. IEEE Transactions on Software Engineering 20, 5, 385403.

PIOLI, A. 1999. Conditional pointer aliasing and constant propagation. Master's Thesis, SUNY at New Paltz. Available at http://www.mcs .newpaltz/tr as Technical Report 99-102.

Pollock, L. ANd Soffa, M. 1989. An incremental version of iterative data flow analysis. IEEE Transactions on Software Engineering 15, 12.

Polychronopolous, C. D. 1988. Parallel Programming and Compilers. Kluwer Academic Publishers.

Ramalingam, G. 1994. The undecidability of aliasing. ACM Transactions on Programming Languages and Systems 16, 5, 1467-1471.

ACM Transactions on Programming Languages and Systems, Vol. 23, No. 1, March 2001. 
Reps, T. And Rosay, G. 1995. Precise interprocedural chopping. In Proceedings of the Third ACM SIGSOFT Symposium on Foundations of Software Engineeering, pp. 41-52.

Reps, T., Horwitz, S. And Sagiv, M. 1995. Precise interprocedural dataflow analysis via graph reachability. In Conference Record of the Twenty-Second Annual ACM SIGACT/SIGPLAN Symposium on Principles of Programming Languages, pp. 49-61.

Rountev, A. ANd Chandra, S. 2000. Off-line variable substitution for scaling points-to analysis. In Proceedings of the ACM SIGPLAN 'OO Conference on Programming Language Design and Implementation, pp. 47-56.

Rountev, A., Milanova, A., ANd Ryder, B. G. 2000. Points-to analysis for Java using annotated inclusion constraints. To appear in Proceedings of OOPSLA'01: Conference on Object-Oriented Programming Systems, Languages and Applications. Also available as Rutgers University Department of Computer Science Technical Report DCS-TR-428.

Rountev, A., Ryder, B. G., And Land, W. A. 1999. Data-flow analysis of program fragments. In Proceedings of Sixth ACM SIGSOFT Symposium on Foundations of Software Engineering, LNCS 1687, pp. 235-252.

RugGieri, C. ANd Murtagh, T. 1988. Lifetime analysis of dynamically allocated objects. In Conference Record of the Fifteenth Annual ACM SIGACT/SIGPLAN Symposium on Principles of Programming Languages, pp. 285-293.

Rugina, R. AND RinaRd, M. 1999. Pointer analysis for multithreaded programs. In Proceedings of the ACM SIGPLAN '99 Conference on Programming Language Design and Implementation, pp. 77-90.

Ryder, B. G. 1983. Incremental data flow analysis. In Conference Record of the Tenth Annual ACM SIGACT/SIGPLAN Symposium on Principles of Programming Languages, pp. 167-176.

RYder, B. G. AND PAull, M. C. 1988. Incremental data flow analysis algorithms. ACM Transactions on Programming Languages and Systems 10, 1, 1-50.

RUF, E. 1995. Context-insensitive alias analysis reconsidered. In Proceedings of the SIGPLAN '95 Conference on Programming Language Design and Implementation, pp. 13-22.

RuF, E. 1997. Partitioning data flow analysis using types. In Conference Record of the TwentyFourth Annual ACM SIGACT/SIGPLAN Symposium on Principles of Programming Languages, pp. 15-26.

Sagiv, S., Francez, N., Rodeh, M., And Wilhelm, R. 1990. A logic-based approach to data flow analysis. In Proceedings of the Second International Workshop in Programming Language Implementation and Logic Programming. pp. 277-292. Volume 456 of Lecture Notes in Computer Science.

Sagiv, M., RePs, T., ANd Wilhelm, R. 1998. Solving shape-analysis problems in languages with destructive updating. ACM Transactions on Programming Languages and Systems 20, 1, 150 .

Shapiro, M. AND Horwitz, S. 1997a. The effects of the precision of pointer analysis. In Proceedings of the Fourth International Symposium on Static Analysis (SAS'97), pp. 1634.

Shapiro, M. AND Horwitz, S. 1997b. Fast and accurate flow-insensitive points-to analysis. In Conference Record of the Twenty-Fourth Annual ACM SIGACT/SIGPLAN Symposium on Principles of Programming Languages, pp. 1-14.

Sharir, M. ANd PNUEl, A. 1981. Two approaches to interprocedural data flow analysis. In Program Flow Analysis: Theory and Applications, S. Muchnick and N. Jones, Ed., Prentice Hall, Englewood, Cliff., pp. 189-234.

Shivers, O. 1988. Control flow analysis in scheme. In Proceedings of the SIGPLAN'88 Conference on Programming Language Design and Implementation, pp. 164-174.

Sinha, S., Harrold, M. J., AND Rothermel, G. 1999. System-dependence-graph-based slicing of programs with arbitrary interprocedural control flow. In Proceedings of the Twenty-First International Conference on Software Engineering, pp. 432-441.

SPILlman, T. 1971. Exposing side effects in a PL-I optimizing compiler. In Proceedings of IFIPS Conference, pp. TA-3-56-TA-3-62.

SteEnsGaARD, B. 1996a. Points-to analysis by type inference of programs with structures and unions. In Proceedings of the Sixth International Conference on Compiler Construction, pp. 136150. Also available as LNCS 1060. 
SteensgaArd, B. 1996b. Points-to analysis in almost linear time. In Conference Record of the Twenty-Third Annual ACM SIGACT/SIGPLAN Symposium on Principles of Programming Languages, pp. 32-41.

TIP, F. 1996. A survey of program slicing techniques. Journal of Programming Languages 3, 3, pp. $121-189$.

Tip, F., Choi, J.-D., Field, J., and Ramalingam, G. 1996. Slicing class hierarchies in $\mathrm{C}^{++}$. In Proceedings of OOPSLA'96: Conference on Object-Oriented Programming Systems, Languages and Applications, pp. 179-197.

Tonella, P., Antoniol, G., Fiutern, R., and Merlo, E. 1997. Flow-insensitive $\mathrm{C}^{++}$pointers and polymorphism analysis and its application to slicing. In Proceedings of the Nineteenth International Conference on Software Engineering (ICSE97), pp. 433-443.

Venkatesh, G. A. 1991. The semantic approach to program slicing. In Proceedings of the SIGPLAN '91 Conference on Programming Language Design and Implementation, pp. 107-119.

Weinl, W. E. 1980. Interprocedural data flow analysis in the presence of pointers, procedure variables and label variables. Master's Thesis, M.I.T.

WeISER, M. 1984. Program slicing. IEEE Transactions on Software Engineering SE-10, 4, 352357.

WeyUKer, E. 1994. More experience with data flow testing. IEEE Transactions on Software Engineering 19, 9, 912-919.

WILSON, R. AND LAM, M. 1995. Efficient context-sensitive pointer analysis for C programs. In Proceedings of the SIGPLAN '95 Conference on Programming Language Design and Implementation, pp. 1-12. Also available as SIGPLAN Notices, Vol. 30, No. 6.

Wolfe, M. 1989. Optimizing Supercompilers for Supercomputers. The MIT Press, Cambridge, MA.

Yong, S. H., Horwitz, S., And RePs, T. 1999. Pointer analysis for programs with structures and casting. In Proceedings of the ACM SIGPLAN '99 Conference on Programming Language Design and Implementation, pp. 91-103.

YuR, J., RYder, B. G., AND LANDI, W. 1999. An incremental flow- and context-sensitive pointer aliasing analysis. In Proceedings of the Twenty-First International Conference on Software Engineering, pp. 442-451.

Yur, J., Ryder, B. G., LANDI, W., AND Stocks, P. 1997. Incremental analysis of side effects for C software systems. In Proceedings of the Nineteenth International Conference on Software Engineering, pp. 422-432.

ZHANG, S. 1995. Program decomposition. Student poster presentation at PLDI'95.

Zhang, S. 1998. Practical pointer aliasing analyses for C. Ph.D. Thesis, Rugters University. Also available as Dept. of Computer Science Technical Report DCS-TR-367.

Zhang, S., Ryder, B. G., ANd LANDI, W. 1996. Program decomposition for pointer aliasing: A step towards practical analyses. In Proceedings of the Fourth Annual ACM SIGSOFT Symposium on the Foundations of Software Engineering, pp. 81-92.

Zhang, S., Ryder, B. G., AND LANDI, W. A. 1998. Experiments with combined analysis for pointer aliasing. In Proceedings of ACM SIGPLAN Workshop on Program Analysis and Software Tools for Engineering, pp. 11-18.

Received May 1998; revised August 1999 and October 2000; accepted December 2000 